\title{
New a priori and a posteriori probabilistic bounds for robust counterpart optimization: I. Unknown probability distributions
}

\author{
Yannis A. Guzman ${ }^{\mathrm{c}, \mathrm{a}, \mathrm{b}}$, Logan R. Matthews ${ }^{\mathrm{c}, \mathrm{a}, \mathrm{b}}$, Christodoulos A. Floudas ${ }^{\mathrm{a}, \mathrm{b}, *}$ \\ ${ }^{a}$ Artie McFerrin Department of Chemical Engineering, Texas A $\mathscr{G} M$ University, College \\ Station, TX, USA \\ ${ }^{b}$ Texas A\&M Energy Institute, Texas A\&M University, College Station, TX, USA \\ ${ }^{c}$ Department of Chemical and Biological Engineering, Princeton University, Princeton, NJ, \\ $U S A$
}

\begin{abstract}
Optimization problems often have a subset of parameters whose values are not known exactly or have yet to be realized. Nominal solutions to models under uncertainty can be infeasible or yield overly optimistic objective function values given the actual parameter realizations. Worst-case robust optimization guarantees feasibility but yields overly conservative objective function values. The use of probabilistic guarantees greatly improves the performance of robust counterpart optimization. We present new a priori and a posteriori probabilistic bounds which improve upon existing methods applied to models with uncertain parameters whose possible realizations are bounded and subject to unspecified probability distributions. We also provide new a priori and a posteriori bounds which, for the first time, permit robust counterpart optimization of models with parameters whose means are only known to lie within some range of values. The utility of the bounds is demonstrated through computational case studies involving a mixed-integer linear optimization problem and a linear multiperiod planning problem. These bounds reduce the conservatism, improve the performance, and augment the applicability of robust counterpart optimization.
\end{abstract}

\section{Introduction}

The parameters of applied mathematical models often have multiple possible values which they can achieve due to limited information or measurement error. The solutions and objective function values produced from optimizing models with uncertain parameters can vary greatly based on which values the uncertain parameters realize. One method of handling uncertain parameters is to guarantee feasibility of the constraints for all possible parameter points

\footnotetext{
*Corresponding author. Tel.: +1979 4580253.

Email address: floudas@tamu.edu (Christodoulos A. Floudas)
}

Preprint submitted to Elsevier

September 11, 2015 
contained within deterministically defined parameter spaces, called uncertainty sets; the corresponding optimization model is known as a robust counterpart. For bounded uncertainty, that is, for the case where upper and lower bounds on each uncertain parameter are known, Soyster (1973) formulated the so-called "worst-case" robust counterpart, where each constraint's uncertainty set is defined to include all possible parameter points; the probability that parameters would realize values rendering an optimal solution infeasible is zero. El Ghaoui and Lebret (1997) applied robust optimization methods to uncertain leastsquares optimization problems, and El Ghaoui et al. (1998) solved uncertain semidefinite optimization problems.

Less conservative solutions can also be obtained for models with bounded or unbounded uncertain parameters, where the uncertainty sets can be defined with a corresponding probability of constraint violation greater than zero. For bounded uncertainty in linear optimization models (LPs), Ben-Tal and Nemirovski (2000) proposed the interval + ellipsoidal uncertainty set and derived a method to provide an upper bound on constraint violation given the size of the uncertainty set. Bertsimas and Sim (2004) proposed and characterized the interval + polyhedral uncertainty set, which yielded a linear robust counterpart. The robust counterpart optimization framework was extended to mixed-integer linear optimization models (MILPs) by Floudas and coworkers (Janak et al., 2007; Lin et al., 2004; Verderame and Floudas, 2009a,b) with parameters subject to uncertainty with known or unknown distributions, including unbounded probability distributions.

In a traditional robust counterpart optimization framework, the quality of an optimal solution to the robust counterpart relies heavily on a priori methods that define uncertainty sets which are guaranteed to satisfy a particular upper bound on the probability of constraint violation; a tighter probabilistic bound would allow the imposition of a smaller uncertainty set with the same guarantee of feasibility and can drastically improve the objective function values. In other words, a tighter probabilistic upper bound on constraint violation leads to less conservative solutions. Both Ben-Tal and Nemirovski (2000) and Bertsimas and Sim (2004) derived a priori methods that provided an upper bound on the probability of constraint violation based on uncertainty set size for interval + ellipsoidal and interval + polyhedral sets, respectively. Kang et al. (2013) utilized distribution-dependent bounds with the interval + polyhedral uncertainty set. Li et al. (2011) extended these bounds to apply to other uncertainty sets so that a variety of a priori bounds were available for box, ellipsoidal, interval + ellipsoidal, polyhedral, and interval + polyhedral uncertainty sets. An alternative approach is to characterize the probability of constraint violation of a particular solution, that is, to obtain a probabilistic guarantee a posteriori (Kang et al., 2013; Li and Floudas, 2014; Li et al., 2012; Paschalidis et al., 2008), which would yield a tighter bound than an a priori bound of equivalent structure. An a posteriori bound can be incorporated into the robust counterpart, permitting feasibility only when the bound is met, as an alternative to an uncertainty set, though this yields a highly nonconvex optimization problem (Li and Floudas, 2014). Li and Floudas (2014) proposed an iterative 
method which yields better solutions than those obtained from the traditional robust counterpart optimization framework (i.e., defining uncertainty sets via a priori bounds), by converging them towards the tighter results given by $a$ posteriori bounds. Utilizing tight a priori and a posteriori bounds with this iterative method can provide drastically improved solutions when compared to the worst-case approach, as well as the traditional one-pass robust counterpart optimization framework.

We present fundamental theoretical results on new a priori and a posteriori bounds on the probability of constraint violation which improve upon existing methods. Situations for which one or more of the new bounds are applicable include (i) bounded, symmetric or asymmetric uncertain parameters with known means and unknown probability distributions, and (ii) bounded uncertain parameters with limited information on their means. With the proposed a priori and a posteriori bounds, robust counterpart optimization can be applied to models with parameters matching the latter case for the first time. The new $a$ priori bounds yield smaller uncertainty sets while guaranteeing the same probability of constraint violation when compared with existing methods. A new $a$ posteriori bound for symmetric, unspecified probability distributions can yield lower probabilities of constraint violation for the same robust solution. An $a$ posteriori bound given uncertain parameters with limited information on their means is also provided. These methods can provide greatly improved objective function values, both when used within a traditional robust counterpart framework and when applied to the a priori - a posteriori iterative algorithm of Li and Floudas (2014).

\section{Background}

The scope of this work includes uncertain parameters which participate linearly or are coefficients of variables participating linearly in a constraint or objective function. Given continuous variables $x$ and integer variables $y$, consider the nonlinear function $f_{i}(x, y)$ participating in constraint $i$ :

$$
f_{i}(x, y)+\sum_{k} a_{i k} x_{k}+\sum_{\ell} b_{i \ell} y_{i \ell}+\sum_{m} p_{i m} \leq 0 .
$$

Relevant to this work is the case where the exact value of some or all of the parameters $a_{i k}, b_{i \ell}$, and $p_{i m}$ are unknown. Without loss of generality, constraint (1) can be reformulated as:

$$
\begin{aligned}
f_{i}(x, y)+t_{i} & \leq 0 \\
-t_{i}+\sum_{k} a_{i k} x_{k}+\sum_{\ell} b_{i \ell} y_{i \ell}+\sum_{m} p_{i m} & \leq 0 .
\end{aligned}
$$

The same type of reformulation can be applied to an objective function under uncertainty. Thus, we will assume that all uncertain constraints only involve linearly participating variables which are continuous or integer. The typical presentation of robust optimization goes further and assumes that the entire model 
is an LP or MILP. We present the background section under this assumption so as to maintain consistency. The study of uncertainty in parameters that participate nonlinearly in the objective function or constraints is beyond the scope of this paper and will be the subject of future work.

The general form of a LP or MILP under uncertainty is as follows:

$$
\begin{array}{lll}
\max _{x, y} & \sum_{k} \tilde{c}_{k} x_{k}+\sum_{\ell} \tilde{d}_{\ell} y_{\ell} & \\
\text { s.t. } & \sum_{k} \tilde{a}_{i k} x_{k}+\sum_{\ell} \tilde{b}_{i \ell} y_{\ell} \leq \tilde{p}_{i} \quad & \forall i \\
& y_{\ell} \in\{0,1\} & \forall \ell
\end{array}
$$

where any parameter denoted with a tilde, say $\tilde{p}$, denotes a parameter subject to uncertainty. Different realizations of the uncertain parameters can change the solution and objective function value of (3). Model (3) can be equivalently written ( $\mathrm{Li}$ et al., 2012) as:

$$
\begin{array}{lll}
\max _{x, y, z} & z & \\
\text { s.t. } & z-\sum_{k} \tilde{c}_{k} x_{k}-\sum_{\ell} \tilde{d}_{\ell} y_{\ell} \leq 0 & \\
& \tilde{p}_{i} x_{0}+\sum_{k} \tilde{a}_{i k} x_{k}+\sum_{\ell} \tilde{b}_{i \ell} y_{\ell} \leq 0 & \forall i \\
& x_{0}=-1 & \\
& y_{\ell} \in\{0,1\} & \forall \ell .
\end{array}
$$

An uncertain parameter $\tilde{a}$ can be represented by a constant nominal value $a$, which is generally set to $\mathbf{E}[\tilde{a}]$, and a random variable $\xi$ which subjects $a$ to perturbations weighted by a known positive constant $\hat{a}$ :

$$
\tilde{a}=a+\xi \hat{a} .
$$

Without loss of generality, models (3) and (4) can be represented as:

$$
\begin{array}{ll}
\max _{x} & \sum_{j} c_{j} x_{j} \\
\text { s.t. } & \sum_{j} a_{i j} x_{j}+\sum_{j \in J_{i}} \xi_{i j} \hat{a}_{i j} x_{j} \leq b_{i} \quad \forall i
\end{array}
$$

where $x_{j}$ is a continuous or integer variable, and $J_{i}:=\left\{j: \hat{a}_{i j}>0\right\}$ is the set of indices where the coefficients of $x$ in constraint $i$ are subject to uncertainty.

In general, the nominal solution to a model under uncertainty, where each uncertain parameter is held at its nominal value, can only assure feasibility of the model if every uncertain parameter realizes its nominal value. A robust solution to a model under uncertainty will permit uncertain parameters to realize multiple values while remaining feasible. If the nominal value of each uncertain 
parameter is chosen as its expected value, then an optimal solution using the nominal parameter point in parameter space can be considered to be a type of base or average case. One can then extend outwards from the nominal parameter point and require a robust solution to guarantee feasibility for more parameter points in parameter space, thus increasing the robustness of the solution as more points are included. A set of points in parameter space that a robust solution must account for is called an uncertainty set, and is defined separately for each constraint under uncertainty. We denote the uncertainty set of constraint $i$ as $U_{i}$. Given the notation of (6), $U_{i}$ will contain some or all realizations of the random vector $\xi_{i}$ with elements $\xi_{i j}, j \in J_{i}$, including the nominal parameter point, $\xi_{i}=\mathbf{0}$, which corresponds to the nominal solution. The robust counterpart of model (6) can thus be written as

$$
\begin{array}{ll}
\max _{x} & \sum_{j} c_{j} x_{j} \\
\text { s.t. } & \sum_{j} a_{i j} x_{j}+\max _{\xi_{i} \in U_{i}}\left\{\sum_{j \in J_{i}} \xi_{i j} \hat{a}_{i j} x_{j}\right\} \leq b_{i} \quad \forall i .
\end{array}
$$

Uncertainty sets are generally constructed geometrically by including parameter points within some norm distance around the nominal point. The worstcase interval set proposed by Soyster (1973) can be generalized as an $\infty$-norm distance about the nominal point, and is called a box uncertainty set ( $\mathrm{Li}$ et al., 2011):

$$
U_{i}^{\infty}=\left\{\xi_{i}:\left\|\xi_{i}\right\|_{\infty}=\max _{j \in J_{i}}\left|\xi_{i j}\right| \leq \Psi_{i}\right\}
$$

Similarly, the 1-norm or 2-norm can be used, yielding the polyhedral and ellipsoidal uncertainty set, respectively (Ben-Tal and Nemirovski, 1998, 1999, 2000; Bertsimas and Sim, 2004; El Ghaoui and Lebret, 1997; El Ghaoui et al., 1998; Li et al., 2011):

$$
\begin{aligned}
& U_{i}^{1}=\left\{\xi_{i}:\left\|\xi_{i}\right\|_{1}=\sum_{j \in J_{i}}\left|\xi_{i j}\right| \leq \Gamma_{i}\right\} \\
& U_{i}^{2}=\left\{\xi_{i}:\left\|\xi_{i}\right\|_{2}=\sqrt{\sum_{j \in J_{i}} \xi_{i j}^{2}} \leq \Omega_{i}\right\} .
\end{aligned}
$$

Note that increasing parameters $\Psi_{i}, \Gamma_{i}$, and $\Omega_{i}$ from zero increases the size of $U_{i}$. We will use the notation $\Delta_{i}$ to denote the size parameter of arbitrary uncertainty set $U_{i}$. If it is assumed that $\xi_{i j} \in[-1,1], \forall j \in J_{i}$, then when $\Psi_{i}=1$, all possible parameter realizations are included in the box uncertainty set (thus becoming the worst-case or interval set). For polyhedral and ellipsoidal sets, this occurs when $\Gamma_{i}=\left|J_{i}\right|$ and $\Omega_{i}=\sqrt{\left|J_{i}\right|}$, respectively (Li et al., 2011). However, with these values of $\Gamma_{i}$ and $\Omega_{i}$, more parameter points than the worstcase set are included due to the geometry of the underlying norm. Ben-Tal and Nemirovski (2000) proposed the interval + ellipsoidal uncertainty set, which is 
the intersection of the interval and ellipsoidal sets and is never larger than the worst-case set:

$$
U_{i}^{2 \cap \infty}=\left\{\xi_{i}:\left\|\xi_{i}\right\|_{2} \leq \Omega_{i},\left\|\xi_{i}\right\|_{\infty} \leq 1\right\} .
$$

Similarly, Bertsimas and Sim (2004) proposed the interval + polyhedral uncertainty set:

$$
U_{i}^{1 \cap \infty}=\left\{\xi_{i}:\left\|\xi_{i}\right\|_{1} \leq \Gamma_{i},\left\|\xi_{i}\right\|_{\infty} \leq 1\right\} .
$$

The five uncertainty sets considered in this work are summarized in Table 4. The introduction of uncertainty sets into a robust counterpart formulation is summarized in Table 5 and discussed in detail in (Li et al., 2011; Li and Floudas, 2014; Li et al., 2012).

If the solution to a model under uncertainty must be feasible for any possible realization of its uncertainty parameters, all of which are bounded, then the robust counterpart should include only interval uncertainty sets $\left(\Psi_{i}=1\right)$, which typically yields very conservative solutions. However, if one or more parameters are unbounded, or if the situation can tolerate some small, nonzero probability that constraint $i$ is infeasible, the objective function value can be drastically improved when compared to the worst-case solution. One way this is accomplished is by reducing the size of $U_{i}$ via selecting $\Delta_{i}$ values that are less than the worst-case value prior to solving the robust counterpart. The size of $U_{i}$ is selected such that any subsequent solution is guaranteed to be feasible in constraint $i$ using any parameter realization with a user-specified probability of $\epsilon_{i}^{\text {prio }}$ or less:

$$
\operatorname{Pr}\left\{\sum_{j} a_{i j} x_{j}+\sum_{j \in J_{i}} \xi_{i j} \hat{a}_{i j} x_{j}>b_{i}\right\} \leq \epsilon_{i}^{\text {prio }} .
$$

Methods which can relate the size of $U_{i}$ to $\epsilon_{i}^{\text {prio }}$ are thus called a priori probabilistic bounds.

Alternatively, a specific solution $x^{*}$ to the robust counterpart can be characterized by determining an upper bound $\epsilon_{i}^{\text {post }}$ on the probability that the solution will be feasible in constraint $i$ with any parameter realization:

$$
\operatorname{Pr}\left\{\sum_{j} a_{i j} x_{j}^{*}+\sum_{j \in J_{i}} \xi_{i j} \hat{a}_{i j} x_{j}^{*}>b_{i}\right\} \leq \epsilon_{i}^{\text {post }} .
$$

Methods which can relate a specific solution of the robust counterpart to the probability of constraint violation are called a posteriori probabilistic bounds.

It is desirable to utilize the tightest a priori or a posteriori bounding methods available; at the same value of $\epsilon_{i}^{\text {prio }}$, a tighter a priori method can yield a better objective function value than a looser method, while a tighter a posteriori method can give a lower estimate of $\epsilon_{i}^{\text {post }}$ for a given solution than a looser method. 


\section{Preliminaries}

Given that, for all $j \in J_{i}$, random variable $\xi_{i j}$ is independent, the following relations for the a priori probability of constraint violation hold for the $i$ th constraint, any uncertainty set $U_{i}$ outlined above, and any $\theta>0$ (Ben-Tal and Nemirovski, 2000; Bertsimas and Sim, 2004; Li et al., 2012):

$$
\begin{aligned}
\operatorname{Pr}\left\{\sum_{j} a_{i j} x_{j}+\sum_{j \in J_{i}} \xi_{i j} \hat{a}_{i j} x_{j}>b_{i}\right\} & \leq \operatorname{Pr}\left\{\sum_{j \in J_{i}} \delta_{i j} \xi_{i j}>\Delta_{i}\right\} \\
& \leq e^{-\theta \Delta_{i}} \prod_{j \in J_{i}} \mathbf{E}\left[e^{\theta \delta_{i j} \xi_{i j}}\right]
\end{aligned}
$$

where the definitions of $\delta_{i j}$ are dependent on the classification of uncertainty set $U_{i}$ and are given in Table 6. Each $\delta_{i j} \in[-1,1]$ and is essentially $\hat{a}_{i j} x_{j}$ scaled by a factor related to $U_{i}$; for more details, see (Li et al., 2012). The a priori value of $\delta_{i j}$ is not known; the full range and combination of values of elements $\delta_{i j}, \forall j \in J_{i}$, of vector $\delta_{i}$ must be taken into account. Based on the type of uncertainty set $U_{i}$, the various norm distances of the vector $\delta_{i}$ are constrained to be less than or equal to different values. Parameter $\Delta_{i}$ represents size parameter $\Psi_{i}, \Omega_{i}$, or $\Gamma_{i}$ for box, ellipsoidal/interval + ellipsoidal, or polyhedral/interval + polyhedral uncertainty sets, respectively (see Tables 4 and 5). Relation (11) follows from Markov's inequality and independence of $\xi_{i j}$. Relations (10) and (11) will be used as starting points for some of the theorems in Section 4.

We also require three lemmas. The first was noted by Hoeffding (1963) but is not the well-known Hoeffding's lemma, though it is tighter. It can be seen as a specific case of Bennett's Lemma where the standard deviation of the probability distribution is not known (Bennett, 1962; Dembo and Zeitouni, 2009).

Lemma 1. Given the random variable $\xi$ is subject to a probability distribution that is supported on the bounded interval $[a, b]$, and given that its moment generating function $M_{\xi}(\theta)=\mathbf{E}\left[e^{\theta \xi}\right]$ exists, then for any real number $\theta$,

$$
\mathbf{E}\left[e^{\theta \xi}\right] \leq \frac{b-\mathbf{E}[\xi]}{b-a} e^{\theta a}+\frac{\mathbf{E}[\xi]-a}{b-a} e^{\theta b}
$$

Proof. The function $\exp (\theta \xi)$ is convex in $\xi$. Thus,

$$
\exp \left(\theta\left((1-\lambda) \xi_{1}+\lambda \xi_{2}\right)\right) \leq(1-\lambda) e^{\theta \xi_{1}}+\lambda e^{\theta \xi_{2}} \quad \forall \xi_{1}, \xi_{2} \in[a, b], \forall \lambda \in[0,1] .
$$

Taking $\xi_{1}=a, \xi_{2}=b$, and $(1-\lambda) \xi_{1}+\lambda \xi_{2}=\xi$, the relation becomes

$$
e^{\theta \xi} \leq \frac{b-\xi}{b-a} e^{\theta a}+\frac{\xi-a}{b-a} e^{\theta b} \quad \forall \xi \in[a, b] .
$$


Therefore,

$$
\mathbf{E}\left[e^{\theta \xi}\right] \leq \mathbf{E}\left[\frac{b-\xi}{b-a} e^{\theta a}+\frac{\xi-a}{b-a} e^{\theta b}\right]=\frac{b-\mathbf{E}[\xi]}{b-a} e^{\theta a}+\frac{\mathbf{E}[\xi]-a}{b-a} e^{\theta b} .
$$

Solely by applying Lemma 1, better a priori bounds can be derived. However, bounds can be further tightened by considering the natural upper bound on $\left\|\delta_{i}\right\|_{1}$, denoted as $\delta_{i}^{(1)}$, which depends on the uncertainty set type (see Table 6).

Lemma 2. Let $f_{j}: X \rightarrow Y$, where $X=\left\{x_{j} \in \mathbb{R}: 0 \leq x^{L} \leq x_{j} \leq x^{U}\right\}$ and $Y=$ $\{y \in \mathbb{R}: 0 \leq y<\infty\}$, be nondecreasing and log-convex over $X, j=1,2, \ldots, n$, with

$$
\frac{f_{1}\left(x_{i}\right)}{f_{1}\left(x_{j}\right)} \geq \frac{f_{2}\left(x_{i}\right)}{f_{2}\left(x_{j}\right)} \geq \cdots \geq \frac{f_{n}\left(x_{i}\right)}{f_{n}\left(x_{j}\right)} \quad \forall(i, j): x_{i}, x_{j} \in X, x_{i} \geq x_{j} .
$$

Given that the nonconvex optimization problem

$$
\max _{x}\left\{\prod_{j=1}^{n} f_{j}\left(x_{j}\right): \sum_{j=1}^{n} x_{j} \leq x^{\sigma} ; x_{j} \in X, \forall j\right\},
$$

is feasible (i.e., $\left.x^{L} \leq x^{\sigma} / n\right)$, then the point $x^{*}=\left(x_{1}^{*}, x_{2}^{*}, \ldots, x_{j}^{*}, \ldots, x_{n}^{*}\right)^{\top}$, where

$$
\begin{aligned}
x_{j}^{*} & = \begin{cases}x^{U} & j \leq \bar{j} \\
x^{m} & j=\bar{j}+1 \\
x^{L} & j \geq \bar{j}+2,\end{cases} \\
\bar{j} & :=\left\lfloor\frac{x^{\sigma}-n x^{L}}{x^{U}-x^{L}}\right\rfloor, \\
x^{m} & :=x^{\sigma}-\bar{j}\left(x^{U}-x^{L}\right)-(n-1) x^{L},
\end{aligned}
$$

is a global maximizer to problem (P1).

Proof. Note that the codomain of $f_{j}\left(x_{j}\right)$ over $X$ is nonnegative. Then a global minimizer of the following optimization problem:

$$
\min _{x}\left\{-\sum_{j=1}^{n} \ln f_{j}\left(x_{j}\right): \sum_{j=1}^{n} x_{j} \leq x^{\sigma} ; x_{j} \in X, \forall j\right\},
$$

which is a concave minimization problem over a polytope, will be a global maximizer of problem (P1). Furthermore, at least one of the vertices of the polytope will be a global minimizer of problem (13) (Falk and Hoffman, 1976; Tardella, 2003). 
If $x^{L}=x^{\sigma} / n$, the only feasible solution is $x_{j}^{\prime}=x^{L}, \forall j$. Then $\bar{j}=0$, $x^{m}=x^{L}$, and $x_{j}^{\prime}=x_{j}^{*}, \forall j$.

If $x^{U} \leq x^{\sigma} / n$, then the constraint $\sum_{j=1}^{n} x_{j} \leq x^{\sigma}$ is redundant. As $f_{j}(x)$ is nondecreasing, a global minimizer is $x_{j}^{\prime}=x^{U}, \forall j$. Then $\bar{j} \geq n$ and $x_{j}^{\prime}=x_{j}^{*}, \forall j$.

If $x^{L}<x^{\sigma} / n<x^{U}$, the feasible region has a nonempty interior and the constraint $\sum_{j=1}^{n} x_{j} \leq x^{\sigma}$ is not redundant. If this constraint is inactive $\left(\sum_{j=1}^{n} x_{j}<\right.$ $\left.x^{\sigma}\right)$, then there exists some $x_{j}<x^{U}$ which, if increased, would not increase the value of the objective function of (13) given that every $f_{j}\left(x_{j}\right)$ is nondecreasing over $X$. Thus, the set of vertex points where the equality $\sum_{j=1}^{n} x_{j}=x^{\sigma}$ holds contains a global minimizer.

Define the set of indices denoting variables which are on the interior of their interval bounds as

$$
I:=\left\{j: x^{L}<x_{j}^{\prime}<x^{U}\right\} .
$$

If a given feasible point $x^{\prime}$ involves two or more variables which are on the interior of their interval bounds, i.e., $|I|>1$, then $x^{\prime}$ is not a vertex point, as it can be written as a convex combination of two other feasible points $x^{a}$ and $x^{b}$ where an increase in $x_{i}^{a}, x_{j}^{b}, i \neq j, i \in I, j \in I$ can correspond to a decrease in $x_{j}^{a}, x_{i}^{b}$. However, with $|I| \leq 1$, point $x^{\prime}$ cannot be written as a convex combination of two other feasible points and thus is vertex point. Depending on $x^{L}, x^{U}$, and $x^{\sigma}$, vertices where $\sum_{j=1}^{n} x_{j}=x^{\sigma}$ holds will have either one index in $I$ or no indices in $I$.

Consider the weighted sum of two values $x^{L}$ and $x^{U}$, where the sum of the weights equals $n$ and the weighted sum equals $x^{\sigma}$ :

$$
\alpha x_{U}+(n-\alpha) x^{L}=x^{\sigma} .
$$

Solving for $\alpha$ yields

$$
\alpha=\frac{x^{\sigma}-n x^{L}}{x^{U}-x^{L}} .
$$

Note that $\alpha$ is not necessarily an integer amount. Relating this situation to the proof, the floor of $\alpha$ represents the number of variables at $x^{U}$. If $\alpha-\bar{j}=0$, then $|I|=0$ and $x^{m}=x^{L}$. Otherwise, $\alpha-\bar{j}>0,|I|=1$, and it must hold that

$$
x_{\bar{j}+1}=x^{\sigma}-\bar{j} x^{U}-(n-\bar{j}-1) x^{L}=x^{m}
$$

for $\sum_{j=1}^{n} x_{j}=x^{\sigma}$ to hold. In both cases, $x_{j}=x_{j}^{*}, \forall j$.

By the definition of $x^{*}$, the ordering by value of $x_{j}^{*}$ in $x^{*}$ is $x_{1}^{*} \geq x_{2}^{*} \geq \cdots \geq$ $x_{n}^{*}$. Assume that switching some $x_{i}^{*}$ with $x_{k}^{*}, i<k, x_{i}>x_{k}$, would improve the 
objective function of (13). This means

$$
\begin{aligned}
& -\sum_{j \notin\{i, k\}} \ln f_{j}\left(x_{j}\right)-\ln f_{i}\left(x_{k}\right)-\ln f_{k}\left(x_{i}\right) \\
& <-\sum_{j \notin\{i, k\}} \ln f_{j}\left(x_{j}\right)-\ln f_{i}\left(x_{i}\right)-\ln f_{k}\left(x_{k}\right) \quad x_{i}>x_{k} \\
& \rightarrow-\ln f_{i}\left(x_{k}\right)-\ln f_{k}\left(x_{i}\right)<-\ln f_{i}\left(x_{i}\right)-\ln f_{k}\left(x_{k}\right) \quad x_{i}>x_{k} \\
& \rightarrow \quad \ln \left(f_{i}\left(x_{k}\right) f_{k}\left(x_{i}\right)\right)>\ln \left(f_{i}\left(x_{i}\right) f_{k}\left(x_{k}\right)\right) \quad x_{i}>x_{k} \\
& \rightarrow \quad f_{i}\left(x_{k}\right) f_{k}\left(x_{i}\right)>f_{i}\left(x_{i}\right) f_{k}\left(x_{k}\right) \quad x_{i}>x_{k} \\
& \rightarrow \quad \frac{f_{k}\left(x_{i}\right)}{f_{k}\left(x_{k}\right)}>\frac{f_{i}\left(x_{i}\right)}{f_{i}\left(x_{k}\right)} \quad x_{i}>x_{k}
\end{aligned}
$$

which contradicts conditions (12).

Remark 1. If functions $\ln f_{j}\left(x_{j}\right)$ are differentiable over $X$, conditions (12) are equivalent to

$$
\begin{aligned}
& \frac{f_{1}\left(x_{i}\right)}{f_{1}\left(x_{j}\right)} \geq \cdots \geq \frac{f_{n}\left(x_{i}\right)}{f_{n}\left(x_{j}\right)} \quad \forall(i, j): x_{i}, x_{j} \in X, x_{i} \geq x_{j} \\
& \rightarrow \quad \ln f_{1}\left(x_{i}\right)-\ln f_{1}\left(x_{j}\right) \geq \cdots \\
& \geq \ln f_{n}\left(x_{i}\right)-\ln f_{n}\left(x_{j}\right) \quad \forall(i, j): x_{i}, x_{j} \in X, x_{i} \geq x_{j} \\
& \rightarrow \quad \int_{x_{j}}^{x_{i}} \frac{d \ln f_{1}(x)}{d x} d x \geq \cdots \\
& \geq \int_{x_{j}}^{x_{i}} \frac{d \ln f_{n}(x)}{d x} d x \quad \forall(i, j): x_{i}, x_{j} \in X, x_{i} \geq x_{j},
\end{aligned}
$$

which implies

$$
\frac{d \ln f_{1}(x)}{d x} \geq \cdots \geq \frac{d \ln f_{n}(x)}{d x} \quad \forall x \in X
$$

as alternate conditions to conditions (12) which can be used to qualify Lemma 2.

Remark 2. If $x^{L}=0$ and $x^{U}=1, \bar{j}=\left\lfloor x^{\sigma}\right\rfloor$. Using $\left|\delta_{i j}\right|$ for $x_{j}\left(\left|\delta_{i j}\right| \in[0,1]\right)$, $\bar{j}=\left\lfloor\delta_{i}^{(1)}\right\rfloor$.

For box, polyhedral, and interval + polyhedral sets, any vector $\delta_{i}$ which satisfies the constraint $\left\|\delta_{i}\right\|_{1} \leq \delta_{i}^{(1)}$ will also satisfy the the constraint $\left\|\delta_{i}\right\|_{2} \leq$ $\delta_{i}^{(2)}$, where $\delta_{i}^{(2)}$ is the natural upper bound on $\left\|\delta_{i}\right\|_{2}$. However, for ellipsoidal and interval + ellipsoidal sets, the latter constraint is both an equality and more restrictive than the former; vectors $\delta_{i}$ which satisfy the 1-norm constraint can violate the 2 -norm constraint. The following holds for any vector $x \in \mathbb{R}^{n}$ due to the Cauchy-Schwarz inequality:

$$
\|x\|_{1} \leq n^{1 / 2}\|x\|_{2} .
$$


Thus, because $\delta_{i}^{(1)}=\left|J_{i}\right|^{1 / 2} \delta_{i}^{(2)}$ for ellipsoidal and interval + ellipsoidal sets, we only need to consider the constraint on $\left\|\delta_{i}\right\|_{2}$.

Lemma 3. Let $f: X \rightarrow Y$, where $X=\{x \in \mathbb{R}: 0 \leq x \leq 1\}$ and $Y=\{y \in \mathbb{R}$ : $0<y<\infty\}$, be nondecreasing over $X$, and let the composite function $(f \circ g)(x)$, where $g(x)=\sqrt{x}$, be concave over $X$. Then the optimization problem

$$
\max _{x}\left\{\sum_{j} f\left(x_{j}\right): \sum_{j} x_{j}^{2} \leq 1 ; x_{j} \in[0,1], \forall j\right\}
$$

with $j=1,2, \ldots, n$, has an optimal solution $x_{j}^{*}=1 / \sqrt{n}$

Proof. With a change of variables $w_{j}=x_{j}^{2}$, problem (P2) is equivalent to

$$
\max _{w}\left\{\sum_{j} f\left(\sqrt{w_{j}}\right): \sum_{j} w_{j} \leq 1 ; w_{j} \in[0,1], \forall j\right\},
$$

which is a concave maximization over a convex set. Because the function $f\left(x_{j}\right)$ is nondecreasing over the domain, the function $f\left(\sqrt{w_{j}}\right)$ is also nondecreasing over the domain. Thus, the region where the equality $\sum_{j} w_{j}=1$ holds will contain an optimal solution. At any feasible point $w^{*}$ where the equality holds, if there exists some $w_{i}^{*}<w_{k}^{*}, i \neq k$, an increase in $w_{i}^{*}$ (and a corresponding decrease in $w_{k}^{*}$ to satisfy $\left.\sum_{j} w_{j}=1\right)$ will not decrease the objective function value due to the concavity of $f\left(\sqrt{w_{j}}\right)$. Thus, an optimal solution to (15) is $w_{j}^{*}=1 / n, \forall j$, corresponding to $x_{j}^{*}=1 / \sqrt{n}, \forall j$.

\section{A Priori Bounds}

We organize the a priori bounds by the amount of information that is known about the uncertain parameters in constraint $i$, that is, assumptions on their means and whether they are subject to a symmetric distribution. Existing $a$ priori bounds and new a priori bounds proposed in this work are summarized in Tables 7 and 8, and their comparisons are summarized in Table 9.

\subsection{Uncertain parameters are bounded and have bounded means}

In this case, the uncertain parameters have known lower and upper bounds, and their means are known only to within some range of values, that is, a single expected value cannot be confidently imposed. Instead of the nominal value of $\tilde{a}_{i j}$ representing the mean, yielding $\mathbf{E}\left[\xi_{i j}\right]=0$, the nominal value is chosen such that $\left|\mathbf{E}\left[\xi_{i j}\right]\right| \leq \mu_{i j}$. To our knowledge, this is the first a priori bound that applies to this case.

Theorem 1. Given that, for all $j \in J_{i}$, random variable $\xi_{i j}$ is independent and subject to a probability distribution that is supported on the bounded interval 
$[-1,1]$, and given that $\left|\mathbf{E}\left[\xi_{i j}\right]\right| \leq \mu_{i j}$, the following probability bound of constraint violation holds for the ith constraint with $\Delta_{i}>\sum_{k \in K_{i}} \mu_{i k}$ and any $\theta>0$ :

$$
\begin{array}{r}
\operatorname{Pr}\left\{\sum_{j} a_{i j} x_{j}+\sum_{j \in J_{i}} \xi_{i j} \hat{a}_{i j} x_{j}>b_{i}\right\} \\
\leq \exp \left(\min _{\theta>0}\left\{-\theta \Delta_{i}+\sum_{k \in K_{i}} \ln G_{i k}(\theta)\right\}\right)
\end{array}
$$

where $G_{i j}(\theta):=\mu_{i j} \sinh (\theta)+\cosh (\theta)$ and $K_{i}$ contains the indices of the $\left\lceil\delta_{i}^{(1)}\right\rceil$ largest $\mu_{i j}$. For $0 \leq \Delta_{i} \leq \sum_{k \in K_{i}} \mu_{i k}$, the bound approaches a probability of 1 as $\theta$ approaches 0 . The parameter $\delta_{i}^{(1)}$ depends on $U_{i}$ and is given in Table 6 .

Proof.

$$
\begin{aligned}
\operatorname{Pr}\left\{\sum_{j} a_{i j} x_{j}+\sum_{j \in J_{i}} \xi_{i j} \hat{a}_{i j} x_{j}>b_{i}\right\} & \\
& \leq e^{-\theta \Delta_{i}} \prod_{j \in J_{i}} \mathbf{E}\left[e^{\left.\theta \delta_{i j} \xi_{i j}\right]}\right. \\
& \leq e^{-\theta \Delta_{i}} \prod_{j \in J_{i}}\left(\frac{1-\mathbf{E}\left[\xi_{i j}\right]}{2} e^{-\theta \delta_{i j}}+\frac{\mathbf{E}\left[\xi_{i j}\right]+1}{2} e^{\theta \delta_{i j}}\right)
\end{aligned}
$$

where relation 1 is due to Lemma 1 . Note that $\mathbf{E}\left[\xi_{i j}\right] \in[-1,1]$; the term within the product operator is nonnegative. If $\delta_{i j} \geq 0$, then $\exp \left(\theta \delta_{i j}\right) \geq \exp \left(-\theta \delta_{i j}\right)$, $\forall x \geq 0$, and the expression is maximized when $\mathbf{E}\left[\xi_{i j}\right]=\mu_{i j}$. If $\delta_{i j} \leq 0$, then $\exp \left(\theta \delta_{i j}\right) \leq \exp \left(-\theta \delta_{i j}\right), \forall x \geq 0$, and the expression is maximized when $\mathbf{E}\left[\xi_{i j}\right]=$ $-\mu_{i j}$. Thus,

$$
\frac{1-\mathbf{E}\left[\xi_{i j}\right]}{2} e^{-\theta \delta_{i j}}+\frac{\mathbf{E}\left[\xi_{i j}\right]+1}{2} e^{\theta \delta_{i j}} \leq \frac{1-\mu_{i j}}{2} e^{-\theta\left|\delta_{i j}\right|}+\frac{\mu_{i j}+1}{2} e^{\theta\left|\delta_{i j}\right|} .
$$

Then,

$$
\begin{aligned}
e^{-\theta \Delta_{i}} \prod_{j \in J_{i}}\left(\frac{1-\mathbf{E}\left[\xi_{i j}\right]}{2} e^{-\theta \delta_{i j}}\right. & \left.+\frac{\mathbf{E}\left[\xi_{i j}\right]+1}{2} e^{\theta \delta_{i j}}\right) \\
& \stackrel{2}{\leq} e^{-\theta \Delta_{i}} \prod_{j \in J_{i}}\left(\frac{1-\mu_{i j}}{2} e^{-\theta\left|\delta_{i j}\right|}+\frac{\mu_{i j}+1}{2} e^{\theta\left|\delta_{i j}\right|}\right) \\
& =e^{-\theta \Delta_{i}} \prod_{j \in J_{i}}\left(\mu_{i j} \sinh \left(\theta\left|\delta_{i j}\right|\right)+\cosh \left(\theta\left|\delta_{i j}\right|\right)\right) \\
& =e^{-\theta \Delta_{i}} \prod_{j \in J_{i}} G_{i j}\left(\theta\left|\delta_{i j}\right|\right) .
\end{aligned}
$$


where relation 2 is due to (16).

The optimization problem

$$
\max _{\left|\delta_{i j}\right|}\left\{\prod_{j \in J_{i}} G_{i j}\left(\theta\left|\delta_{i j}\right|\right):\left\|\delta_{i j}\right\|_{1} \leq \delta_{i}^{(1)} ;\left|\delta_{i j}\right| \in[0,1], \forall j \in J_{i}\right\}
$$

matches (P1) of Lemma 2. Ordering $G_{i j}\left(\theta\left|\delta_{i j}\right|\right)$ by decreasing $\mu_{i j}$ assures conditions (14) are satisfied:

$$
\begin{aligned}
& \frac{d \ln G_{i 1}\left(\theta\left|\delta_{i j}\right|\right)}{d\left|\delta_{i j}\right|} \geq \frac{d \ln G_{i 2}\left(\theta\left|\delta_{i j}\right|\right)}{d\left|\delta_{i j}\right|} \quad \forall\left|\delta_{i j}\right| \in[0,1], \forall \theta \geq 0 \\
& \rightarrow \quad \theta \frac{\mu_{i 1} \cosh \left(\theta \delta_{i j}\right)+\sinh \left(\theta \delta_{i j}\right)}{\mu_{i 1} \sinh \left(\theta \delta_{i j}\right)+\cosh \left(\theta \delta_{i j}\right)} \\
& \geq \theta \frac{\mu_{i 2} \cosh \left(\theta \delta_{i j}\right)+\sinh \left(\theta \delta_{i j}\right)}{\mu_{i 2} \sinh \left(\theta \delta_{i j}\right)+\cosh \left(\theta \delta_{i j}\right)} \quad \forall\left|\delta_{i j}\right| \in[0,1], \forall \theta \geq 0 \\
& \rightarrow \quad \mu_{i 1}\left(\cosh ^{2}\left(\theta \delta_{i j}\right)-\sinh ^{2}\left(\theta \delta_{i j}\right)\right) \\
& \geq \mu_{i 2}\left(\cosh ^{2}\left(\theta \delta_{i j}\right)-\sinh ^{2}\left(\theta \delta_{i j}\right)\right) \quad \forall\left|\delta_{i j}\right| \in[0,1], \forall \theta \geq 0 \\
& \rightarrow \quad \mu_{i 1} \geq \mu_{i 2} \text {. }
\end{aligned}
$$

Then,

$$
\begin{aligned}
e^{-\theta \Delta_{i}} \prod_{j \in J_{i}} G_{i j}\left(\theta\left|\delta_{i j}\right|\right) & \\
& \stackrel{3}{\leq} e^{-\theta \Delta_{i}}\left(\prod_{k \in K_{i} \backslash\{\bar{j}\}} G_{i k}(\theta)\right) G_{i \bar{j}}\left(\theta\left[\delta_{i}^{(1)}-\left\lfloor\delta_{i}^{(1)}\right]\right]\right) \\
& =\exp \left(-\theta \Delta_{i}+\sum_{k \in K_{i} \backslash\{\bar{j}\}} \ln G_{i k}(\theta)+\ln G_{i \bar{j}}\left(\theta\left[\delta_{i}^{(1)}-\left\lfloor\delta_{i}^{(1)}\right\rfloor\right]\right)\right) \\
& \left.\leq \sum_{k \in K_{i} \backslash\{\bar{j}\}} \ln G_{i k}(\theta)+\left(\delta_{i}^{(1)}-\left\lfloor\delta_{i}^{(1)}\right\rfloor\right) \ln G_{i \bar{j}}(\theta)\right) \\
& \leq \exp \left(-\theta \Delta_{i}+\sum_{k \in K_{i}} \ln G_{i k}(\theta)\right)
\end{aligned}
$$

where relation 3 is due to Lemma 2 , and relation 4 is due to convexity of $\ln G_{i j}(\theta)$ and the fact that $\left(\delta_{i}^{(1)}-\left\lfloor\delta_{i}^{(1)}\right\rfloor\right) \in[0,1)$. The tightest bound is obtained by minimizing the expression over $\theta>0$.

We can solve for the allowable range of $\Delta_{i}$ by determining when $\theta^{*}=0$ after 
imposing first-order optimality conditions:

$$
\begin{aligned}
\left.\frac{d}{d \theta}\left(-\theta \Delta_{i}+\sum_{k \in K_{i}} \ln G_{i k}(\theta)\right)\right|_{\theta=0} & \\
& =\left[-\Delta_{i}+\sum_{k \in K_{i}} \frac{\mu_{i k} \cosh (\theta)+\sinh (\theta)}{\mu_{i k} \sinh (\theta)+\cosh (\theta)}\right]_{\theta=0} \\
& =-\Delta_{i}+\sum_{k \in K_{i}} \mu_{i k}=\left.0\right|_{\theta=\theta^{*}} \\
& \rightarrow \Delta_{i}=\sum_{k \in K_{i}} \mu_{i k}
\end{aligned}
$$

Example 1. Consider the constraint

$$
\widetilde{1} x_{1}+\widetilde{1} x_{2} \leq \widetilde{20}
$$

where parameters $\widetilde{1}, \widetilde{1}$, and $\widetilde{20}$ are subject to bounded uncertainty. Each parameter can realize values within $10 \%$ of their nominal values 1,1 , and 20 , respectively, and can be written as:

$$
\begin{aligned}
\widetilde{20} & =20+2 \xi_{0} \\
\widetilde{1} & =1+0.1 \xi_{1} \\
\widetilde{1} & =1+0.1 \xi_{2},
\end{aligned}
$$

where random variable $\xi_{j} \in[-1,1], j=0,1,2$. Constraint (17) can be reformulated as:

$$
\begin{aligned}
x_{1}+x_{2}+2 \xi_{0} x_{0}+0.1 \xi_{1} x_{1}+0.1 \xi_{2} x_{2} & \leq 20 \\
x_{0} & =-1 .
\end{aligned}
$$

The expected values of the parameters are only known to be within $1 \%$ of their nominal values:

$$
\begin{gathered}
\mathbf{E}[\widetilde{20}] \in[19.8,20.2] \rightarrow \mathbf{E}\left[\xi_{0}\right] \in[-0.1,0.1] \rightarrow\left|\mathbf{E}\left[\xi_{0}\right]\right| \leq 0.1=\mu_{0} \\
\mathbf{E}[\widetilde{1}] \in[0.99,1.01] \rightarrow \mathbf{E}\left[\xi_{1}\right] \in[-0.1,0.1] \rightarrow\left|\mathbf{E}\left[\xi_{1}\right]\right| \leq 0.1=\mu_{1} \\
\mathbf{E}[\widetilde{1}] \in[0.99,1.01] \rightarrow \mathbf{E}\left[\xi_{2}\right] \in[-0.1,0.1] \rightarrow\left|\mathbf{E}\left[\xi_{2}\right]\right| \leq 0.1=\mu_{2} .
\end{gathered}
$$

A probability of constraint violation of 0.2 or less will be met using bound (GMF1) and an interval + polyhedral set. Thus, $\left|K_{i}\right|=\left\lceil\delta_{i}^{(1)}\right\rceil=3$, and $K_{i}=\{0,1,2\}$. The bisection method with bound (GMF1) can be performed to determine the value of $\Delta_{i}$ (that is, $\Gamma$ ) which will guarantee the desired probability of constraint violation (see Section 6 for details). Selecting $\Delta_{i}=\Gamma=2.930$ 
and using bound (GMF1):

$$
\exp \left(\min _{\theta>0}\{-2.930 \theta+3 \ln (0.1 \sinh (\theta)+\cosh (\theta))\}\right)=\exp (-1.61)=0.20,
$$

where $\theta^{*}=2.119$. As per Table 5 , the robust counterpart of the constraint with an interval + polyhedral set is:

$$
\begin{aligned}
x_{1}+x_{2}+p_{0}+p_{1}+p_{2}+2.930 z & \leq 20 \\
z+p_{0} & \geq 2 \\
z+p_{1} & \geq 0.1\left|x_{1}\right| \\
z+p_{2} & \geq 0.1\left|x_{2}\right| \\
z, p_{0}, p_{1}, p_{2} & \geq 0 .
\end{aligned}
$$

Corollary 1.1. For ellipsoidal and interval + ellipsoidal uncertainty sets, the following probability bound of constraint violation holds for the ith constraint with $\Delta_{i}>\bar{\mu}_{i} \sqrt{\left|J_{i}\right|}$ and any $\theta>0$ :

$$
\begin{aligned}
\operatorname{Pr}\left\{\sum_{j} a_{i j} x_{j}+\sum_{j \in J_{i}} \xi_{i j} \hat{a}_{i j} x_{j}>b_{i}\right\} & \\
\leq & \exp \left(\min _{\theta>0}\left\{-\theta \Delta_{i}+\left|J_{i}\right| \ln \bar{G}_{i}\left(\theta / \sqrt{\left|J_{i}\right|}\right)\right\}\right)
\end{aligned}
$$

where $\bar{\mu}_{i}=\max _{j \in J_{i}} \mu_{i j}$ and $\bar{G}_{i}(\theta):=\bar{\mu}_{i} \sinh (\theta)+\cosh (\theta)$. For $0 \leq \Delta_{i} \leq$ $\bar{\mu}_{i} \sqrt{\left|J_{i}\right|}$, the bound approaches a probability of 1 as $\theta$ approaches 0.

Proof.

$$
\begin{aligned}
\operatorname{Pr}\left\{\sum_{j} a_{i j} x_{j}+\sum_{j \in J_{i}} \xi_{i j} \hat{a}_{i j} x_{j}>b_{i}\right\} & \\
& \leq e^{-\theta \Delta_{i}} \prod_{j \in J_{i}} \mathbf{E}\left[e^{\left.\theta \delta_{i j} \xi_{i j}\right]}\right. \\
& \leq e^{-\theta \Delta_{i}} \prod_{j \in J_{i}}\left(\frac{1-\mathbf{E}\left[\xi_{i j}\right]}{2} e^{-\theta \delta_{i j}}+\frac{\mathbf{E}\left[\xi_{i j}\right]+1}{2} e^{\theta \delta_{i j}}\right) \\
& \leq e^{-\theta \Delta_{i}} \prod_{j \in J_{i}}\left(\frac{1-\bar{\mu}_{i}}{2} e^{-\theta\left|\delta_{i j}\right|}+\frac{\bar{\mu}_{i}+1}{2} e^{\theta\left|\delta_{i j}\right|}\right) \\
& =e^{-\theta \Delta_{i}} \prod_{j \in J_{i}} \bar{G}_{i}\left(\theta\left|\delta_{i j}\right|\right) \\
& =\exp \left(-\theta \Delta_{i}+\sum_{j \in J_{i}} \ln \bar{G}_{i}\left(\theta\left|\delta_{i j}\right|\right)\right)
\end{aligned}
$$


where relation 1 is due to Lemma 1, and relation 2 is to (16).

The optimization prob

$$
\max _{\left|\delta_{i j}\right|}\left\{\sum_{j \in J_{i}} \ln \bar{G}_{i}\left(\theta\left|\delta_{i j}\right|\right):\left\|\delta_{i j}\right\|_{2} \leq \delta_{i}^{(2)} ;\left|\delta_{i j}\right| \in[0,1], \forall j \in J_{i}\right\}
$$

matches problem (P2) of Lemma 3. The conditions of Lemma 3 require concavity of $\bar{G}_{i}\left(\theta \sqrt{\left|\delta_{i j}\right|}\right)$. With $z_{i j}:=2 \theta \sqrt{\left|\delta_{i j}\right|}$, it must hold that

$$
\begin{array}{rlrl}
\frac{d^{2} \bar{G}_{i}\left(\theta \sqrt{\left|\delta_{i j}\right|}\right)}{d\left|\delta_{i j}\right|} \leq 0 & \forall\left|\delta_{i j}\right| \in[0,1], \forall \theta \geq 0 \\
\rightarrow \quad-\theta\left[\left(2+\bar{\mu}_{i}^{2}\right) \sinh \left(z_{i j}\right)+2 \bar{\mu}_{i} \cosh \left(z_{i j}\right)\right. & \\
\left.\rightarrow \quad-\left(1-\bar{\mu}_{i}^{2}\right) z_{i j}\right] /\left[8\left|\delta_{i j}\right|^{3 / 2}\left(\bar{G}_{i}\left(\theta \sqrt{\left|\delta_{i j}\right|}\right)\right)^{2}\right] & \leq 0 & \forall\left|\delta_{i j}\right| \in[0,1], \forall \theta \geq 0 \\
\rightarrow \quad\left(2+\bar{\mu}_{i}^{2}\right) \sinh \left(z_{i j}\right)+2 \bar{\mu}_{i} \cosh \left(z_{i j}\right)-\left(1-\bar{\mu}_{i}^{2}\right) z_{i j} \geq 0 & \forall z_{i j} \geq 0 \\
\rightarrow \quad \frac{\left(1+\bar{\mu}_{i}\right)^{2}}{2} e^{z_{i j}}-\frac{\left(1-\bar{\mu}_{i}\right)^{2}}{2} e^{-z_{i j}}-\left(1-\bar{\mu}_{i}^{2}\right) z_{i j} \geq 0 & \forall z_{i j} \geq 0 .
\end{array}
$$

The final relation is nondecreasing as $\bar{\mu}_{i}$ increases for any $z_{i j}$, and is thus minimized at $\bar{\mu}_{i}=0$ :

$$
\sinh \left(z_{i j}\right)-z_{i j} \geq 0 \quad \bar{\mu}_{i}=0, \forall z_{i j} \geq 0
$$

which holds true.

Thus,

$$
\exp \left(-\theta \Delta_{i}+\sum_{j \in J_{i}} \ln \bar{G}_{i}\left(\theta\left|\delta_{i j}\right|\right)\right) \stackrel{3}{\leq} \exp \left(-\theta \Delta_{i}+\left|J_{i}\right| \bar{G}_{i}\left(\theta / \sqrt{\left|J_{i}\right|}\right)\right)
$$

where relation 3 is due to Lemma 3 . The tightest bound is obtained by minimizing the expression over $\theta>0$. As in Theorem 1 , first-order optimality conditions yield the ranges of $\Delta_{i}$.

Example 2. Consider the constraint

$$
\widetilde{1} x_{1}+\widetilde{1} x_{2} \leq \widetilde{20}
$$

where parameters $\widetilde{1}, \widetilde{1}$, and $\widetilde{20}$ are subject to bounded uncertainty. Each parameter can realize values within $10 \%$ of their nominal values 1,1 , and 20 , respectively, and can be written as:

$$
\begin{aligned}
\widetilde{20} & =20+2 \xi_{0} \\
\widetilde{1} & =1+0.1 \xi_{1} \\
\widetilde{1} & =1+0.1 \xi_{2},
\end{aligned}
$$


where random variable $\xi_{j} \in[-1,1], j=0,1,2$. Constraint (20) can be reformulated as:

$$
\begin{aligned}
x_{1}+x_{2}+2 \xi_{0} x_{0}+0.1 \xi_{1} x_{1}+0.1 \xi_{2} x_{2} & \leq 20 \\
x_{0} & =-1 .
\end{aligned}
$$

The expected values of the parameters are only known to be within $1 \%$ of their nominal values:

$$
\begin{gathered}
\mathbf{E}[\widetilde{20}] \in[19.8,20.2] \rightarrow \mathbf{E}\left[\xi_{0}\right] \in[-0.1,0.1] \rightarrow\left|\mathbf{E}\left[\xi_{0}\right]\right| \leq 0.1=\mu_{0} \\
\mathbf{E}[\widetilde{1}] \in[0.99,1.01] \rightarrow \mathbf{E}\left[\xi_{1}\right] \in[-0.1,0.1] \rightarrow\left|\mathbf{E}\left[\xi_{1}\right]\right| \leq 0.1=\mu_{1} \\
\mathbf{E}[\widetilde{1}] \in[0.99,1.01] \rightarrow \mathbf{E}\left[\xi_{2}\right] \in[-0.1,0.1] \rightarrow\left|\mathbf{E}\left[\xi_{2}\right]\right| \leq 0.1=\mu_{2} .
\end{gathered}
$$

A probability of constraint violation of 0.2 or less will be met using bound $\left(\right.$ GMF1 $\left.^{\prime}\right)$ and an interval + ellipsoidal set. The bisection method with bound (GMF1') can be performed to determine the value of $\Delta_{i}$ (that is, $\Omega$ ) which will guarantee the desired probability of constraint violation (see Section 6 for details). Selecting $\Delta_{i}=\Omega=1.692$ and using bound $\left(\mathrm{GMF}^{\prime}\right)$ :

$$
\begin{aligned}
\exp \left(\min _{\theta>0}\{-1.692 \theta+3 \ln (0.1 \sinh (\theta / \sqrt{3})+\cosh (\theta / \sqrt{3}))\}\right) \\
=\exp (-1.61)=0.20
\end{aligned}
$$

where $\theta^{*}=3.679$. As per Table 5 , the robust counterpart of the constraint with an interval + ellipsoidal set is:

$$
\begin{aligned}
x_{1}+x_{2}+2 \mid-1- & z_{0}|+0.1| x_{1}-z_{1} \mid \\
+ & 0.1\left|x_{2}-z_{2}\right|+1.692 \sqrt{4 z_{0}^{2}+0.01 z_{1}^{2}+0.01 z_{2}^{2}} \leq 20 .
\end{aligned}
$$

\subsection{Uncertain parameters are bounded and have known means}

In this case, the uncertain parameters have known lower and upper bounds, and their means are known. The bounds in this section are included in Table 7. Without loss of generality, we assume uncertain parameter $\tilde{a}_{i j}$ is represented as in (5) with $\xi_{i j} \in[-1,1]$ and $\mathbf{E}\left[\xi_{i j}\right]=0$. Existing bounds for this case are provided by Ben-Tal and Nemirovski (2000) and Bertsimas and Sim (2004), respectively:

$$
\begin{aligned}
& \operatorname{Pr}\left\{\sum_{j} a_{i j} x_{j}+\sum_{j \in J_{i}} \xi_{i j} \hat{a}_{i j} x_{j}>b_{i}\right\} \leq \exp \left(\frac{-\Delta_{i}^{2}}{2}\right), \\
& \operatorname{Pr}\left\{\sum_{j} a_{i j} x_{j}+\sum_{j \in J_{i}} \xi_{i j} \hat{a}_{i j} x_{j}>b_{i}\right\} \leq \exp \left(\frac{-\Delta_{i}^{2}}{2\left|J_{i}\right|}\right) .
\end{aligned}
$$

Bound (BN) was derived for interval + ellipsoidal uncertainty sets and can also be applied to ellipsoidal and box uncertainty sets but not polyhedral and interval 
+ polyhedral uncertainty sets (Li et al., 2012). Bound (BS1) was derived for interval + polyhedral uncertainty sets and can be applied to the other four sets as well (Li et al., 2012), though it is looser than (BN) for box, ellipsoidal, and interval + ellipsoidal sets. While the derivations of these two bounds utilized the conditions that the underlying probability distributions must be symmetric, we note that they can both be derived using Hoeffding's inequality (Hoeffding, 1963) and thus do not require symmetry as a condition.

Theorem 2. Given that, for all $j \in J_{i}$, random variable $\xi_{i j}$ is independent and subject to a probability distribution that is supported on the bounded interval $[-1,1]$ with $\mathbf{E}\left[\xi_{i j}\right]=0$, the following probability bound of constraint violation holds for the ith constraint:

$$
\operatorname{Pr}\left\{\sum_{j} a_{i j} x_{j}+\sum_{j \in J_{i}} \xi_{i j} \hat{a}_{i j} x_{j}>b_{i}\right\} \leq\left(\alpha^{\alpha} \beta^{\beta}\right)^{-\delta_{i}^{(1)} / 2}
$$

where $\alpha:=1-\Delta_{i} / \delta_{i}^{(1)}$ and $\beta:=1+\Delta_{i} / \delta_{i}^{(1)}$. The parameter $\delta_{i}^{(1)}$ depends on $U_{i}$ and is given in Table 6 .

Proof. This is a special case of Theorem 1 with $\mu_{i j}=0, \forall j \in J_{i}$. Starting from the penultimate relation in Theorem 1 ,

$$
\begin{aligned}
\operatorname{Pr}\left\{\sum_{j} a_{i j} x_{j}+\sum_{j \in J_{i}} \xi_{i j} \hat{a}_{i j} x_{j}>b_{i}\right\} \\
\leq \exp \left(-\theta \Delta_{i}+\sum_{k \in K_{i} \backslash\{\bar{j}\}} \ln G_{i k}(\theta)+\left(\delta_{i}^{(1)}-\left\lfloor\delta_{i}^{(1)}\right\rfloor\right) \ln G_{i \bar{j}}(\theta)\right) \\
\quad=\exp \left(-\theta \Delta_{i}+\left\lfloor\delta_{i}^{(1)}\right\rfloor \ln \cosh (\theta)+\left(\delta_{i}^{(1)}-\left\lfloor\delta_{i}^{(1)}\right\rfloor\right) \ln \cosh (\theta)\right) \\
=\exp \left(-\theta \Delta_{i}+\delta_{i}^{(1)} \ln \cosh (\theta)\right) .
\end{aligned}
$$

The tightest bound is obtained by minimizing the expression over $\theta>0$. By first-order optimality conditions, the following must hold:

$$
\theta^{*}=\operatorname{artanh}\left(\Delta_{i} / \delta_{i}^{(1)}\right)=\frac{1}{2} \ln \left(1+\Delta_{i} / \delta_{i}^{(1)}\right)-\frac{1}{2} \ln \left(1-\Delta_{i} / \delta_{i}^{(1)}\right) .
$$


Thus,

$$
\begin{aligned}
& \exp \left(\operatorname { m i n } _ { \theta > 0 } \left\{-\theta \Delta_{i}+\right.\right.\left.\left.\delta_{i}^{(1)} \ln \cosh (\theta)\right\}\right) \\
&=\exp (-\frac{1}{2} \Delta_{i} \ln \left(1+\Delta_{i} / \delta_{i}^{(1)}\right)+\frac{1}{2} \Delta_{i} \ln \left(1-\Delta_{i} / \delta_{i}^{(1)}\right) \\
&\left.\quad-\frac{1}{2} \delta_{i}^{(1)} \ln \left(1+\Delta_{i} / \delta_{i}^{(1)}\right)-\frac{1}{2} \delta_{i}^{(1)} \ln \left(1-\Delta_{i} / \delta_{i}^{(1)}\right)\right) \\
&= \exp \left(\ln \left(1+\Delta_{i} / \delta_{i}^{(1)}\right)^{-\frac{1}{2} \delta_{i}^{(1)}\left(1+\Delta_{i} / \delta_{i}^{(1)}\right)}\right) \\
&\left.\quad+\ln \left(1-\Delta_{i} / \delta_{i}^{(1)}\right)^{-\frac{1}{2} \delta_{i}^{(1)}\left(1-\Delta_{i} / \delta_{i}^{(1)}\right)}\right) \\
&=\left(\left(1+\Delta_{i} / \delta_{i}^{(1)}\right)^{\left(1+\Delta_{i} / \delta_{i}^{(1)}\right)}\left(1-\Delta_{i} / \delta_{i}^{(1)}\right)^{\left(1-\Delta_{i} / \delta_{i}^{(1)}\right)}\right)^{-\delta_{i}^{(1)} / 2} .
\end{aligned}
$$

Example 3. Consider the constraint

$$
\widetilde{1} x_{1}+\widetilde{2} x_{2} \leq \widetilde{12}
$$

where parameters $\widetilde{1}, \widetilde{2}$, and $\widetilde{12}$ are subject to bounded uncertainty. Each parameter can realize values within $10 \%$ of their nominal values 1,2 , and 12 , respectively, and can be written as:

$$
\begin{aligned}
\widetilde{12} & =12+1.2 \xi_{0} \\
\widetilde{1} & =1+0.1 \xi_{1} \\
\widetilde{2} & =2+0.2 \xi_{2},
\end{aligned}
$$

where random variable $\xi_{j} \in[-1,1], j=0,1,2$. Constraint (23) can be reformulated as:

$$
\begin{aligned}
x_{1}+2 x_{2}+1.2 \xi_{0} x_{0}+0.1 \xi_{1} x_{1}+0.2 \xi_{2} x_{2} & \leq 12 \\
x_{0} & =-1 .
\end{aligned}
$$

The nominal value of each parameter was chosen as its expected value; therefore, $\mathbf{E}\left[\xi_{j}\right]=0, j=0,1,2$. A probability of constraint violation of 0.2 or less will be met using bound (GMF2) and an interval + polyhedral set. Thus, $\delta_{i}^{(1)}=$ 3. The bisection method with bound (GMF2) can be performed to determine the value of $\Delta_{i}$ (that is, $\Gamma$ ), which will guarantee the desired probability of constraint violation (see Section 6 for details). Selecting $\Delta_{i}=\Gamma=2.781$ and 
using bound (GMF2):

$$
\begin{aligned}
& \alpha=1-\frac{2.781}{3}=0.073 \\
& \beta=1+\frac{2.781}{3}=1.927 \\
& {\left[(0.073)^{(0.073)}(1.927)^{(1.927)}\right]^{-3 / 2}=0.20 .}
\end{aligned}
$$

As per Table 5, the robust counterpart of the constraint with an interval + polyhedral set is:

$$
\begin{aligned}
x_{1}+2 x_{2}+p_{0}+p_{1}+p_{2}+2.781 z & \leq 12 \\
z+p_{0} & \geq 1.2 \\
z+p_{1} & \geq 0.1\left|x_{1}\right| \\
z+p_{2} & \geq 0.2\left|x_{2}\right| \\
z, p_{0}, p_{1}, p_{2} & \geq 0 .
\end{aligned}
$$

Corollary 2.1. For ellipsoidal and interval + ellipsoidal uncertainty sets, the following probability bound of constraint violation holds for the ith constraint:

$$
\operatorname{Pr}\left\{\sum_{j} a_{i j} x_{j}+\sum_{j \in J_{i}} \xi_{i j} \hat{a}_{i j} x_{j}>b_{i}\right\} \leq\left(\alpha^{\alpha} \beta^{\beta}\right)^{-\left|J_{i}\right| / 2}
$$

where $\alpha:=1-\Delta_{i} / \delta_{i}^{(1)}$ and $\beta:=1+\Delta_{i} / \delta_{i}^{(1)}$. The parameter $\delta_{i}^{(1)}$ depends on $U_{i}$ and is given in Table 6 .

Proof. This is a special case of Corollary 1.1 with $\bar{\mu}_{i}=0$. Starting from $\left(\mathrm{GMF}^{\prime}\right)$,

$$
\begin{aligned}
\operatorname{Pr}\left\{\sum_{j} a_{i j} x_{j}+\sum_{j \in J_{i}} \xi_{i j} \hat{a}_{i j} x_{j}>b_{i}\right\} & \\
\leq & \exp \left(\min _{\theta>0}\left\{-\theta \Delta_{i}+\left|J_{i}\right| \ln \bar{G}_{i}\left(\theta / \sqrt{\left|J_{i}\right|}\right)\right\}\right) \\
& =\exp \left(\min _{\theta>0}\left\{-\theta \Delta_{i}+\left|J_{i}\right| \ln \cosh \left(\theta / \sqrt{\left|J_{i}\right|}\right)\right\}\right) .
\end{aligned}
$$

By first-order optimality conditions, the following must hold:

$$
\begin{aligned}
\theta^{*}=\sqrt{\left|J_{i}\right|} \operatorname{artanh}\left(\Delta_{i} /\right. & \left.\sqrt{\left|J_{i}\right|}\right)=\sqrt{\left|J_{i}\right|} \operatorname{artanh}\left(\Delta_{i} / \delta_{i}^{(1)}\right) \\
= & \frac{1}{2} \sqrt{\left|J_{i}\right|} \ln \left(1+\Delta_{i} / \delta_{i}^{(1)}\right)-\frac{1}{2} \sqrt{\left|J_{i}\right|} \ln \left(1-\Delta_{i} / \delta_{i}^{(1)}\right) .
\end{aligned}
$$


Thus,

$$
\begin{aligned}
\exp \left(\min _{\theta>0}\left\{-\theta \Delta_{i}+\left|J_{i}\right| \ln \cosh \left(\theta / \sqrt{\left|J_{i}\right|}\right)\right\}\right) \\
=\exp \left(-\frac{1}{2} \sqrt{\left|J_{i}\right|} \Delta_{i} \ln \left(1+\Delta_{i} / \delta_{i}^{(1)}\right)+\frac{1}{2} \sqrt{\left|J_{i}\right|} \Delta_{i} \ln \left(1-\Delta_{i} / \delta_{i}^{(1)}\right)\right. \\
\left.\quad-\frac{1}{2}\left|J_{i}\right| \ln \left(1+\Delta_{i} / \delta_{i}^{(1)}\right)-\frac{1}{2}\left|J_{i}\right| \ln \left(1-\Delta_{i} / \delta_{i}^{(1)}\right)\right) \\
=\exp \left(\ln \left(1+\Delta_{i} / \delta_{i}^{(1)}\right)^{-\frac{1}{2}\left|J_{i}\right|\left(1+\Delta_{i} / \delta_{i}^{(1)}\right)}\right. \\
\left.\quad+\ln \left(1-\Delta_{i} / \delta_{i}^{(1)}\right)^{-\frac{1}{2}\left|J_{i}\right|\left(1-\Delta_{i} / \delta_{i}^{(1)}\right)}\right) \\
=\left(\left(1+\Delta_{i} / \delta_{i}^{(1)}\right)^{\left(1+\Delta_{i} / \delta_{i}^{(1)}\right)}\left(1-\Delta_{i} / \delta_{i}^{(1)}\right)^{\left(1-\Delta_{i} / \delta_{i}^{(1)}\right)}\right)^{-\left|J_{i}\right| / 2} .
\end{aligned}
$$

Example 4. Consider the constraint

$$
\widetilde{1} x_{1}+\widetilde{2} x_{2} \leq \widetilde{12}
$$

where parameters $\widetilde{1}, \widetilde{2}$, and $\widetilde{12}$ are subject to bounded uncertainty. Each parameter can realize values within $10 \%$ of their nominal values 1,2 , and 12 , respectively, and can be written as:

$$
\begin{aligned}
\widetilde{12} & =12+1.2 \xi_{0} \\
\widetilde{1} & =1+0.1 \xi_{1} \\
\widetilde{2} & =2+0.2 \xi_{2},
\end{aligned}
$$

where random variable $\xi_{j} \in[-1,1], j=0,1,2$. Constraint (26) can be reformulated as:

$$
\begin{aligned}
x_{1}+2 x_{2}+1.2 \xi_{0} x_{0}+0.1 \xi_{1} x_{1}+0.2 \xi_{2} x_{2} & \leq 12 \\
x_{0} & =-1 .
\end{aligned}
$$

where random variable $\xi_{j} \in[-1,1], j=0,1,2$. The nominal value of each parameter was chosen as its expected value; therefore, $\mathbf{E}\left[\xi_{j}\right]=0, j=0,1,2$. A probability of constraint violation of 0.2 or less will be met using bound $\left(\mathrm{GMF}^{\prime}\right)$ and an interval + ellipsoidal set. Thus, $\delta_{i}^{(1)}=\sqrt{3}$. The bisection method with bound $\left(\mathrm{GMF}^{\prime}\right)$ can be performed to determine the value of $\Delta_{i}$ (that is, $\Omega$ ) which will guarantee the desired probability of constraint violation 
(see Section 6 for details). Selecting $\Delta_{i}=\Omega=1.606$ and using bound (GMF2'):

$$
\begin{aligned}
& \alpha=1-\frac{1.606}{\sqrt{3}}=0.073 \\
& \beta=1+\frac{1.606}{\sqrt{3}}=1.927 \\
& {\left[(0.073)^{(0.073)}(1.927)^{(1.927)}\right]^{-3 / 2}=0.20 .}
\end{aligned}
$$

As per Table 5, the robust counterpart of the constraint with an interval + ellipsoidal set is:

$$
\begin{aligned}
x_{1}+2 x_{2}+1.2 \mid & -1-z_{0}|+0.1| x_{1}-z_{1} \mid \\
& +0.2\left|x_{2}-z_{2}\right|+1.606 \sqrt{1.44 z_{0}^{2}+0.01 z_{1}^{2}+0.04 z_{2}^{2}} \leq 12 .
\end{aligned}
$$

Remark 1. For box uncertainty sets (where (BN) is as tight as or tighter than (BS1)), bound (GMF2) is as tight as or tighter than (BN). As $\Delta_{i} \rightarrow 1$, bound (GMF2) approaches $2^{-1}$ as opposed to $\sqrt{e}-1(\sqrt{e} \approx 1.65)$.

Remark 2. For ellipsoidal uncertainty sets (where (BN) is as tight as or tighter than (BS1)), bound $\left(\mathrm{GMF}^{\prime}\right)$ is tighter than $(\mathrm{BN})$. As $\Delta_{i} \rightarrow \sqrt{\left|J_{i}\right|}$, bound (GMF2) approaches $2^{-\left|J_{i}\right|}$ as opposed to $\sqrt{e}-\left|J_{i}\right|$.

Remark 3. For polyhedral uncertainty sets (where only (BS1) applies), bound (GMF2) is as tight as or tighter than (BS1). As $\Delta_{i} \rightarrow\left|J_{i}\right|$, bound (GMF2) approaches $2^{-\left|J_{i}\right|}$ as opposed to $\sqrt{e}^{-\left|J_{i}\right|}$.

\subsection{Uncertain parameters are bounded and symmetric}

In this case, the uncertain parameters have known lower and upper bounds and are assumed to be subject to symmetric probability distributions. The bounds of this section are included in Table 8 . The bounds of the previous section (where symmetry was not assumed) apply to this case, as does the following bound provided by Bertsimas and Sim (2004) derived for interval + polyhedral uncertainty sets with $\Gamma_{i} \geq 1$ :

$$
\begin{aligned}
\operatorname{Pr}\left\{\sum_{j} a_{i j} x_{j}+\sum_{j \in J_{i}} \xi_{i j} \hat{a}_{i j} x_{j} \geq b_{i}\right\} & \\
\leq & \frac{1}{2^{n}}\left[(1-(\nu-\lfloor\nu\rfloor))\left(\begin{array}{c}
n \\
\lfloor\nu\rfloor
\end{array}\right)+\sum_{k=\lfloor\nu\rfloor+1}^{n}\left(\begin{array}{l}
n \\
k
\end{array}\right)\right]
\end{aligned}
$$

where $\nu:=\left(\Gamma_{i}+n\right) / 2$ and $n:=\left|J_{i}\right|$. Li et al. (2012) noted that (BS2) can also apply to polyhedral, ellipsoidal, interval + ellipsoidal, and box uncertainty sets, though it has no utility with box uncertainty sets due to it providing a 
probability bound of constraint violation only at $\Psi_{i}=1$ with a value of 0.5 , which is the worst-case model for bounded uncertainty where the probability of constraint violation is 0 .

None of the aforementioned a priori bounds successfully incorporate the fact that an upper bound on the probability of constraint violation in the nominal case $\left(\Delta_{i}=0\right)$ with bounded and symmetric uncertain parameters should be 0.5. The sum of random variables $\sum_{j \in J_{i}} \delta_{i j} \xi_{i j}$ can be considered a single, new random variable $\Xi_{i}$ with an expected value of 0 and a symmetric probability distribution. Then, $\operatorname{Pr}\left\{\Xi_{i}>0\right\}$ is at most 0.5 , corresponding to the case where $\operatorname{Pr}\left\{\Xi_{i}=0\right\}=0$. A higher upper bound is only possible if $\Xi_{i}$ (that is, any $\xi_{i j}$ ) is not symmetric about 0. Presented below, bound (GMF3) is as tight as or tighter than (BS2) for $\Delta_{i} \geq 1$, applies to all $\Delta_{i} \geq 0$ while (BS2) only applies to $\Delta_{i} \geq 1$, and returns 0.5 as the probability bound of constraint violation at $\Delta_{i}=0$.

Theorem 3. Given that, for all $j \in J_{i}$, random variable $\xi_{i j}$ is independent and subject to a symmetric probability distribution that is supported on the bounded interval $[-1,1]$ with $\mathbf{E}\left[\xi_{i j}\right]=0$, the following probability bound of constraint violation holds for the ith constraint:

$$
\operatorname{Pr}\left\{\sum_{j} a_{i j} x_{j}+\sum_{j \in J_{i}} \xi_{i j} \hat{a}_{i j} x_{j}>b_{i}\right\} \leq \frac{1}{2^{\tilde{n}}} \sum_{k=\lceil\tilde{\nu}\rceil}^{\tilde{n}}\left(\begin{array}{l}
\tilde{n} \\
k
\end{array}\right)
$$

where $\tilde{\nu}:=\left(\Delta_{i}+\tilde{n}\right) / 2$ and

$$
\tilde{n}:= \begin{cases}\left|J_{i}\right|+1-\operatorname{sgn} \Delta_{i} & \text { if }\left\lceil\Delta_{i}\right\rceil+\left|J_{i}\right| \text { is even } \\ \left|J_{i}\right|-\operatorname{sgn} \Delta_{i} & \text { if }\left\lceil\Delta_{i}\right\rceil+\left|J_{i}\right| \text { is odd. }\end{cases}
$$

Proof. Denote a Rademacher random variable as $\xi^{(\mathrm{Ra})}$ (that is, $\operatorname{Pr}\left\{\xi^{(\mathrm{Ra})}=\right.$ $-1\}=0.5$ and $\left.\operatorname{Pr}\left\{\xi^{(\mathrm{Ra})}=1\right\}=0.5\right)$, a Bernoulli random variable with $p=0.5$ as $\xi^{(\mathrm{Be})}$, and a binomial random variable with $p=0.5$ as $\xi^{(\mathrm{Bi})}$. Each product $\delta_{i j} \xi_{i j}$ can be considered a new symmetric random variable $\xi_{i j}^{\prime}$ which is supported on $[-1,1]$ and has an expected value of 0 . First, for $\Delta_{i}>0$, 


$$
\begin{aligned}
& \operatorname{Pr}\left\{\sum_{j \in J_{i}} \delta_{i j} \xi_{i j}>\Delta_{i}\right\}=\operatorname{Pr}\left\{\sum_{j \in J_{i}} \xi_{i j}^{\prime}>\Delta_{i}\right\} \\
& \leq \operatorname{Pr}\left\{\sum_{j \in J_{i}} \xi_{i j}^{\prime} \geq \Delta_{i}\right\} \\
& \stackrel{1}{\leq} \begin{cases}\operatorname{Pr}\left\{\sum_{j=1}^{\left|J_{i}\right|} \xi_{i j}^{(\mathrm{Ra})} \geq \Delta_{i}\right\} & \text { if }\left\lceil\Delta_{i}\right\rceil+\left|J_{i}\right| \text { is even } \\
\operatorname{Pr}\left\{\sum_{j=1}^{\left|J_{i}\right|-1} \xi_{i j}^{(\mathrm{Ra})} \geq \Delta_{i}\right\} & \text { if }\left\lceil\Delta_{i}\right\rceil+\left|J_{i}\right| \text { is odd }\end{cases} \\
& \stackrel{2}{=} \operatorname{Pr}\left\{\sum_{j=1}^{\tilde{n}} \xi_{i j}^{(\mathrm{Ra})} \geq \Delta_{i}\right\} \\
& \stackrel{3}{=} \operatorname{Pr}\left\{\sum_{j=1}^{\tilde{n}}\left(2 \xi_{i j}^{(\mathrm{Be})}-1\right) \geq \Delta_{i}\right\} \\
& =\operatorname{Pr}\left\{\sum_{j=1}^{\tilde{n}} \xi_{i j}^{(\mathrm{Be})} \geq \tilde{\nu}\right\} \\
& \stackrel{4}{=} \operatorname{Pr}\left\{\xi_{i}^{(\mathrm{Bi})} \geq \tilde{\nu}\right\} \\
& =\operatorname{Pr}\left\{\xi_{i}^{(\mathrm{Bi})}=\tilde{\nu}\right\}+\operatorname{Pr}\left\{\xi_{i}^{(\mathrm{Bi})}>\tilde{\nu}\right\} \\
& =\operatorname{Pr}\left\{\xi_{i}^{(\mathrm{Bi})}=\tilde{\nu}\right\}+1-\operatorname{Pr}\left\{\xi_{i}^{(\mathrm{Bi})} \leq \tilde{\nu}\right\} \\
& =\operatorname{Pr}\left\{\xi_{i}^{(\mathrm{Bi})}=\tilde{\nu}\right\}+1-\frac{1}{2^{\tilde{n}}} \sum_{k=0}^{\lfloor\tilde{\nu}\rfloor}\left(\begin{array}{l}
\tilde{n} \\
k
\end{array}\right) \\
& =\operatorname{Pr}\left\{\xi_{i}^{(\mathrm{Bi})}=\tilde{\nu}\right\}+\frac{1}{2^{\tilde{n}}} \sum_{k=\lfloor\tilde{\nu}\rfloor+1}^{\tilde{n}}\left(\begin{array}{l}
\tilde{n} \\
k
\end{array}\right)
\end{aligned}
$$

where relation 1 is due to Theorem 1.1 in (Dzindzalieta et al., 2012), relation 2 is by definition of $\tilde{n}$ with $\operatorname{sgn} \Delta_{i}=1$, relation 3 is due to the relationship between Bernoulli and Rademacher random variables, and relation 4 is due to the relationship between a sum of i.i.d. Bernoulli random variables and the binomial distribution. Note that, in the last expression, the first term, $\operatorname{Pr}\left\{\xi_{i}^{(\mathrm{Bi})}=\tilde{\nu}\right\}$, is only defined for integer $\tilde{\nu}$. If $\tilde{\nu}$ is not integer, then the first 
term is zero and

$$
\frac{1}{2^{\tilde{n}}} \sum_{k=\lfloor\tilde{\nu}\rfloor+1}^{\tilde{n}}\left(\begin{array}{l}
\tilde{n} \\
k
\end{array}\right)=\frac{1}{2^{\tilde{n}}} \sum_{k=\lceil\tilde{\nu}\rceil}^{\tilde{n}}\left(\begin{array}{l}
\tilde{n} \\
k
\end{array}\right) .
$$

Otherwise, if $\tilde{\nu}$ is integer,

$$
\begin{aligned}
\operatorname{Pr}\left\{\xi_{i}^{(\mathrm{Bi})}=\tilde{\nu}\right\}+\frac{1}{2^{\tilde{n}}} \sum_{k=\lfloor\tilde{\nu}\rfloor+1}^{\tilde{n}}\left(\begin{array}{l}
\tilde{n} \\
k
\end{array}\right) & =\frac{1}{2^{\tilde{n}}}\left(\begin{array}{l}
\tilde{n} \\
\tilde{\nu}
\end{array}\right)+\frac{1}{2^{\tilde{n}}} \sum_{k=\tilde{\nu}+1}^{\tilde{n}}\left(\begin{array}{l}
\tilde{n} \\
k
\end{array}\right) \\
& =\frac{1}{2^{\tilde{n}}} \sum_{k=\tilde{\nu}}^{n}\left(\begin{array}{l}
\tilde{n} \\
k
\end{array}\right) \\
& =\frac{1}{2^{\tilde{n}}} \sum_{k=\lceil\tilde{\nu}\rceil}^{\tilde{n}}\left(\begin{array}{l}
\tilde{n} \\
k
\end{array}\right) .
\end{aligned}
$$

Finally, we will show that the bound equals 0.5 for $\Delta_{i} \in[0,1]$. First note that, regardless of $\Delta_{i}, \tilde{n} \geq 1$ for nonempty $J_{i}$, and recall the identity

$$
\sum_{k=0}^{n}\left(\begin{array}{l}
n \\
k
\end{array}\right)=2^{n}
$$

For odd $n$, (29) contains an even number of terms which have a 1-to-1 correspondence for $k<n / 2$ and $k>n / 2$, i.e.,

$$
\left(\begin{array}{c}
n \\
k_{1}
\end{array}\right)=\left(\begin{array}{c}
n \\
k_{2}
\end{array}\right) \quad k_{1}=n-k_{2}, k_{2}=0,1, \ldots,\left\lfloor\frac{n}{2}\right\rfloor .
$$

Say $\Delta_{i} \in(0,1]\left(\operatorname{sgn} \Delta_{i}=1\right)$. Then $\tilde{n}$ is always odd:

$$
\begin{aligned}
\left|J_{i}\right| \text { even } & \rightarrow 1+\left|J_{i}\right| \text { odd } \rightarrow \tilde{n}=\left|J_{i}\right|-1 \text { odd } \\
\left|J_{i}\right| \text { odd } & \rightarrow 1+\left|J_{i}\right| \text { even } \rightarrow \tilde{n}=\left|J_{i}\right| \text { odd },
\end{aligned}
$$

and

$$
\lceil\tilde{\nu}\rceil=\left\lceil\frac{\Delta_{i}}{2}+\frac{\tilde{n}}{2}\right\rceil=\left\lceil\frac{\tilde{n}}{2}\right\rceil .
$$

Thus,

$$
\frac{1}{2^{\tilde{n}}} \sum_{k=\left\lceil\frac{\tilde{n}}{2}\right\rceil}^{\tilde{n}}\left(\begin{array}{l}
\tilde{n} \\
k
\end{array}\right)=\frac{1}{2^{\tilde{n}}}\left(\frac{1}{2} 2^{\tilde{n}}\right)=\frac{1}{2} .
$$

Say $\Delta_{i}=0\left(\operatorname{sgn} \Delta_{i}=0\right)$. Then $\tilde{n}$ is always odd:

$$
\begin{aligned}
\left|J_{i}\right| \text { even } & \rightarrow 0+\left|J_{i}\right| \text { even } \rightarrow \tilde{n}=\left|J_{i}\right|+1 \text { odd } \\
\left|J_{i}\right| \text { odd } & \rightarrow 0+\left|J_{i}\right| \text { odd } \rightarrow \tilde{n}=\left|J_{i}\right| \text { odd }
\end{aligned}
$$


and

$$
\lceil\tilde{\nu}\rceil=\left\lceil\frac{\tilde{n}}{2}\right\rceil .
$$

Once again,

$$
\frac{1}{2^{\tilde{n}}} \sum_{k=\left\lceil\frac{\tilde{n}}{2}\right\rceil}^{\tilde{n}}\left(\begin{array}{l}
\tilde{n} \\
k
\end{array}\right)=\frac{1}{2} .
$$

Example 5. Consider the constraint

$$
\widetilde{1} x_{1}-\widetilde{1} x_{2} \leq-\widetilde{4}
$$

where parameters $\widetilde{1},-\widetilde{1}$, and $-\widetilde{4}$ are subject to bounded and symmetric uncertainty. Each parameter can realize values within $10 \%$ of their nominal values 1, -1 , and -4 , respectively, and can be written as:

$$
\begin{aligned}
-\widetilde{4} & =-4+0.4 \xi_{0} \\
\widetilde{1} & =1+0.1 \xi_{1} \\
-\widetilde{1} & =-1+0.1 \xi_{2},
\end{aligned}
$$

where random variable $\xi_{j} \in[-1,1], j=0,1,2$. Constraint (30) can be reformulated as:

$$
\begin{aligned}
x_{1}-x_{2}+0.4 \xi_{0} x_{0}+0.1 \xi_{1} x_{1}+0.1 \xi_{2} x_{2} & \leq-4 \\
x_{0} & =-1 .
\end{aligned}
$$

where random variable $\xi_{j} \in[-1,1], j=0,1,2$. The nominal value of each parameter was chosen as its expected value; therefore, $\xi_{j}, j=0,1,2$, is symmetric about 0 . A probability of constraint violation of 0.2 or less will be met using bound (GMF3) and an interval + polyhedral set. The bisection method with bound (GMF3) can be performed to determine the value of $\Delta_{i}$ (that is, $\Gamma$ ) which will guarantee the desired probability of constraint violation (see Section 6 for details). Selecting $\Delta_{i}=\Gamma=2.001$ and using bound (GMF3):

$$
\begin{aligned}
& \lceil 2.001\rceil+3=6 \text { (even) } \\
& \tilde{n}=3+1-1=3 \\
& \tilde{\nu}=\frac{2.001+3}{2}=2.5005 \\
& \frac{1}{2^{\tilde{n}}} \sum_{k=\lceil 2.5005\rceil}^{3}\left(\begin{array}{l}
3 \\
k
\end{array}\right)=\frac{1}{8}\left(\begin{array}{l}
3 \\
3
\end{array}\right)=0.125 .
\end{aligned}
$$

Note that with $\Gamma=2.000$, the probability bound of constraint violation is 0.25 , which is larger than $\epsilon_{i}^{\text {prio }}$. As per Table 5, the robust counterpart of the 
constraint with an interval + polyhedral set is:

$$
\begin{aligned}
x_{1}-x_{2}+p_{0}+p_{1}+p_{2}+2.001 z & \leq-4 \\
z+p_{0} & \geq 0.4 \\
z+p_{1} & \geq 0.1\left|x_{1}\right| \\
z+p_{2} & \geq 0.1\left|x_{2}\right| \\
z, p_{0}, p_{1}, p_{2} & \geq 0 .
\end{aligned}
$$

As proposed by Bertsimas and Sim (2004), the term $1 / 2^{n}$ and the binomial coefficient pose computational difficulties at very large $\left|J_{i}\right|$, but can be replaced by the function $C(n, k)$, defined below, with a slight loss of tightness:

$$
\begin{aligned}
\operatorname{Pr}\left\{\sum_{j} a_{i j} x_{j}+\sum_{j \in J_{i}} \xi_{i j} \hat{a}_{i j} x_{j}>b_{i}\right\} & \leq \frac{1}{2^{n}} \sum_{k=\lceil\tilde{\nu}\rceil}^{\tilde{n}}\left(\begin{array}{l}
\tilde{n} \\
k
\end{array}\right) \\
& \leq \sum_{k=\lceil\tilde{\nu}\rceil}^{\tilde{n}} C(\tilde{n}, k)
\end{aligned}
$$

where

$$
C(n, k)= \begin{cases}\frac{1}{2^{n}} & \text { if } k=0 \text { or } k=n \\ \sqrt{\frac{n}{2 \pi(n-k) k}} \exp \left(n \ln \left(\frac{n}{2(n-k)}\right)+k \ln \left(\frac{n-k}{k}\right)\right) & \text { otherwise. }\end{cases}
$$

The discussion in Section 4 included situations where, based on the mathematical structure of the bounds, it can be seen that certain bounds will always be better than other bounds. A more comprehensive overview is given in Table 9 .

\section{A Posteriori Bounds}

Deriving an upper bound on the probability of constraint violation for a given solution (i.e., an a posteriori bound) is easier than deriving one for all possible solutions (i.e., an a priori bound). In principle, an a posteriori bound can follow from the derivation of an a priori bound with specific solution values as opposed to handling all possible values. The a posteriori bound for solution $x^{*}$ in constraint $i$,

$$
\operatorname{Pr}\left\{\sum_{j} a_{i j} x_{j}^{*}+\sum_{j \in J_{i}} \xi_{i j} \hat{a}_{i j} x_{j}^{*}>b_{i}\right\} \leq \epsilon_{i}^{\text {post }},
$$

is equivalent to

$$
\operatorname{Pr}\left\{\sum_{j \in J_{i}} \xi_{i j} \hat{a}_{i j} x_{j}^{*}>h_{i}\left(x^{*}\right)\right\} \leq \epsilon_{i}^{p o s t}
$$


where $h_{i}(x):=b_{i}-\sum_{j} a_{i j} x_{j}$.

We first present the a posteriori analogue to bound (GMF1) for when $\mathbf{E}\left[\xi_{i j}\right]$ is not known exactly.

Theorem 4. Given that, for all $j \in J_{i}$, random variable $\xi_{i j}$ is independent and subject to a probability distribution that is supported on the bounded interval $[-1,1]$, and given that $\left|\mathbf{E}\left[\xi_{i j}\right]\right| \leq \mu_{i j}$, the following probability bound of constraint violation holds for the $i$ th constraint and any $\theta>0$ :

$$
\begin{aligned}
\operatorname{Pr}\left\{\sum_{j} a_{i j} x_{j}^{*}\right. & \left.+\sum_{j \in J_{i}} \xi_{i j} \hat{a}_{i j} x_{j}^{*}>b_{i}\right\} \\
& \leq \exp \left(\min _{\theta>0}\left\{-\theta h_{i}\left(x^{*}\right)+\sum_{j \in J_{i}} \ln G_{i j}\left(\left|\hat{a}_{i j} x_{j}^{*}\right| \theta\right)\right\}\right) .
\end{aligned}
$$

where $G_{i j}(\theta):=\mu_{i j} \sinh (\theta)+\cosh (\theta)$.

Proof. See Appendix A.

As an analogue to the a priori bounds (BN) and (BS1), Li et al. (2012) derived the following a posteriori bound for bounded uncertainty using Hoeffding's inequality (Hoeffding, 1963):

$$
\operatorname{Pr}\left\{\sum_{j} a_{i j} x_{j}^{*}+\sum_{j \in J_{i}} \xi_{i j} \hat{a}_{i j} x_{j}^{*}>b_{i}\right\} \leq \exp \left(\frac{-h_{i}\left(x^{*}\right)^{2}}{2 \sum_{j \in J_{i}} \hat{a}_{i j}^{2} x_{j}^{* 2}}\right) .
$$

Note that the presentation of (LTF) slightly differs from that of Li et al. (2012) due to the assumption without loss of generality that $\mathbf{E}\left[\xi_{i j}\right]=0, \forall j \in J_{i}$.

Just as bounds (GMF2) and (GMF2') improve upon (BN) and (BS1), the following bound by Paschalidis et al. (2008) for bounded uncertainty improves upon (LTF) using an inequality similar to Lemma 1:

$$
\begin{aligned}
\operatorname{Pr}\left\{\sum_{j} a_{i j} x_{j}^{*}+\right. & \left.\sum_{j \in J_{i}} \xi_{i j} \hat{a}_{i j} x_{j}^{*}>b_{i}\right\} \\
& \leq \exp \left(\min _{\theta>0}\left\{-\theta h_{i}\left(x^{*}\right)+\sum_{j \in J_{i}} \ln \cosh \left(\hat{a}_{i j} x_{j}^{*} \theta\right)\right\} .\right.
\end{aligned}
$$

Note that the presentation of (PKL) differs from that of Paschalidis et al. (2008) due to the assumptions that $\mathbf{E}\left[\xi_{i j}\right]=0$ and $\xi_{i j} \in[-1,1]$.

There are two ways to extend the principles of (GMF3) to an a posteriori setting. The proof of the bound requires a sum of bounded and symmetric random variables with expected values of zero and supported within $[-1,1]$. It is applicable to a sum of weighted, symmetric random variables $\xi_{i j} \in[-1,1]$ with $\mathbf{E}\left[\xi_{i j}\right]=0$, so long as their coefficients are also within $[-1,1]$. 
Theorem 5. Given that, for all $j \in J_{i}$, random variable $\xi_{i j}$ is independent and subject to a symmetric probability distribution that is supported on the bounded interval $[-1,1]$ with $\mathbf{E}\left[\xi_{i j}\right]=0$, the following probability bound of constraint violation holds for the ith constraint:

$$
\operatorname{Pr}\left\{\sum_{j} a_{i j} x_{j}^{*}+\sum_{j \in J_{i}} \xi_{i j} \hat{a}_{i j} x_{j}^{*}>b_{i}\right\} \leq \frac{1}{2^{n_{a}}} \sum_{k=\left\lceil\nu_{a}\right\rceil}^{n_{a}}\left(\begin{array}{c}
n_{a} \\
k
\end{array}\right)
$$

where

$$
\begin{aligned}
\nu_{a} & :=\frac{h_{a}+n_{a}}{2} \\
n_{a} & := \begin{cases}\left|J_{i}^{\delta, *}\right|+1-\operatorname{sgn} h_{a} & \text { if }\left\lceil h_{a}\right\rceil+\left|J_{i}^{\delta, *}\right| \text { is even } \\
\left|J_{i}^{\delta, *}\right|-\operatorname{sgn} h_{a} & \text { if }\left\lceil h_{a}\right\rceil+\left|J_{i}^{\delta, *}\right| \text { is odd }\end{cases} \\
h_{a} & :=\frac{\Delta_{i}}{\max _{j \in J_{i}^{\delta, *}}\left|\delta_{i j}^{*}\right|} \\
J_{i}^{\delta, *} & :=\left\{j:\left|\delta_{i j}^{*}\right|>0\right\},
\end{aligned}
$$

and constants $\delta_{i j}^{*}$ are given by their definitions and the solution of the robust counterpart.

Proof. See Appendix A.

Remark 1. Note that (35) would only yield different (and lower) values from (GMF3) if $\max _{j \in J_{i}^{\delta, *}}\left|\delta_{i j}^{*}\right|<1$; other than the trivial case where $\left|J_{i}^{\delta, *}\right|=0$, this does not occur with polyhedral and interval + polyhedral sets.

Bound (35) does not yield the expected reduction in the a posteriori probability when constraint $i$ was inactive in the solution. An alternative is to assure that the coefficients $\hat{a}_{i j} x_{j}^{*}$ on $\xi_{i j}$ are within $[-1,1]$.

Theorem 6. Given that, for all $j \in J_{i}$, random variable $\xi_{i j}$ is independent and subject to a symmetric probability distribution that is supported on the bounded interval $[-1,1]$ with $\mathbf{E}\left[\xi_{i j}\right]=0$, the following probability bound of constraint violation holds for the ith constraint:

$$
\operatorname{Pr}\left\{\sum_{j} a_{i j} x_{j}^{*}+\sum_{j \in J_{i}} \xi_{i j} \hat{a}_{i j} x_{j}^{*}>b_{i}\right\} \leq \frac{1}{2^{n_{b}}} \sum_{k=\left\lceil\nu_{b}\right\rceil}^{n_{b}}\left(\begin{array}{c}
n_{b} \\
k
\end{array}\right)
$$


where

$$
\begin{aligned}
\nu_{b} & :=\frac{h_{b}+n_{b}}{2} \\
n_{b} & := \begin{cases}\left|J_{i}^{*}\right|+1-\operatorname{sgn} h_{b} & \text { if }\left\lceil h_{b}\right\rceil+\left|J_{i}^{*}\right| \text { is even } \\
\left|J_{i}^{*}\right|-\operatorname{sgn} h_{b} & \text { if }\left\lceil h_{b}\right\rceil+\left|J_{i}^{*}\right| \text { is odd }\end{cases} \\
h_{b} & :=\frac{h_{i}\left(x^{*}\right)}{\max _{j \in J_{i}^{*}} \hat{a}_{i j}\left|x_{j}^{*}\right|} \\
J_{i}^{*} & :=\left\{j: \hat{a}_{i j}\left|x_{j}^{*}\right|>0\right\} .
\end{aligned}
$$

Proof. See Appendix A.

Though (36) would on average yield better probabilities than (GMF3) and (35), it is not exactly the analogue of (GMF3), and thus might yield higher $a$ posteriori probabilities. The minimum of the two variants is preferred:

$$
\begin{aligned}
\operatorname{Pr}\left\{\sum_{j \in J_{i}} \xi_{i j} \hat{a}_{i j} x_{j}^{*}>\right. & \left.h_{i}\left(x^{*}\right)\right\} \\
& \leq \min \left\{\frac{1}{2^{n_{a}}} \sum_{k=\left\lceil\nu_{a}\right\rceil}^{n_{a}}\left(\begin{array}{c}
n_{a} \\
k
\end{array}\right), \frac{1}{2^{n_{b}}} \sum_{k=\left\lceil\nu_{b}\right\rceil}^{n_{b}}\left(\begin{array}{c}
n_{b} \\
k
\end{array}\right)\right\} .
\end{aligned}
$$

Note that, for all uncertainty sets but interval + ellipsoidal, the set of indices $J_{i}^{\delta, *}$ is equal to $J_{i}^{*}$, simplifying bound (GMF5) to

$$
\operatorname{Pr}\left\{\sum_{j} a_{i j} x_{j}^{*}+\sum_{j \in J_{i}} \xi_{i j} \hat{a}_{i j} x_{j}^{*}>b_{i}\right\} \leq \frac{1}{2^{n_{c}}} \sum_{k=\left\lceil\nu_{c}\right\rceil}^{n_{c}}\left(\begin{array}{c}
n_{c} \\
k
\end{array}\right)
$$

where $c$ denotes $a$ if $h_{a}>h_{b}$, and $b$ otherwise.

As with (GMF3), the approximation for very high $\left|J_{i}^{\delta, *}\right|$ or $\left|J_{i}^{*}\right|$ is

$$
\begin{aligned}
& \operatorname{Pr}\left\{\sum_{j} a_{i j} x_{j}^{*}+\sum_{j \in J_{i}} \xi_{i j} \hat{a}_{i j} x_{j}^{*}>b_{i}\right\} \\
& \leq \min \left\{\sum_{k=\left\lceil\nu_{a}\right\rceil}^{n_{a}} C\left(n_{a}, k\right), \sum_{k=\left\lceil\nu_{b}\right\rceil}^{n_{b}} C\left(n_{b}, k\right)\right\}
\end{aligned}
$$

where $C(n, k)$ is given in (33).

\section{Implementation}

The a priori bounds presented here yield an upper bound on the probability of constraint violation, $\epsilon_{i}^{\text {prio }}$, for a given $\Delta_{i}$. However, formulating the robust 
counterpart typically seeks the value of $\Delta_{i}$ which will guarantee a particular $\epsilon_{i}^{p r i o}$. A few bounds are able to be analytically rearranged to yield $\Delta_{i}$ as a function of $\epsilon_{i}^{\text {prio }}$. Those which cannot can be easily solved via the bisection method over $\Delta_{i}$ by noting the monotonicity of $\epsilon_{i}^{\text {prio }}$ as a function of $\Delta_{i}$. Complicating matters is the fact that two bounds contain an inner (convex) minimization over $\theta>0$. Performing the bisection method over $\Delta_{i}$ would require solving the minimization at every iteration. However, this is not necessary; the inner minimization can be avoided if the following proposition is applicable.

Proposition 1. Given the NLP

$$
\min _{x}\left\{-c x+f(x): x \geq x^{L}\right\}
$$

where $c>0, f(x)$ is a $\mathcal{C}^{1}$-continuous convex function over $x \geq x^{L}$ with $f\left(x^{L}\right)=c x^{L}$ and $0 \leq d f(x) /\left.d x\right|_{x^{L}}<c$, and $x^{*}$ is an optimal solution, then

(i) $x^{*}>x^{L}$,

(ii) $x^{*}$ is nondecreasing as c increases.

Proof. The conclusions follow from the properties of $f(x)$.

(i) At $x=x^{L}$, the objective function value is 0 . At $x=x^{L}+\epsilon, \epsilon \rightarrow 0^{+}$, the objective function value is decreasing because $c x^{L}+c \epsilon>f\left(x^{L}+\epsilon\right)$. Thus $x^{*}>x^{L}$.

(ii) First order optimality conditions indicate that $c=d f(x) /\left.d x\right|_{x^{*}}$. Due to convexity,

$$
d f(x) /\left.d x\right|_{x_{1}} \geq d f(x) /\left.d x\right|_{x_{2}} \quad \forall x_{1}, x_{2}: x_{1} \geq x_{2} .
$$

Proposition 1 applies to all the bounds presented here with inner minimization problems. The implications of Proposition 1 are that $\epsilon_{i}^{\text {prio }}$ is monotonic in $\theta^{*}$, and that if $\Delta_{i}>0$, constraint $\theta>0$ can be dropped. Then, the inner minimization could be performed using simple algorithms such as a golden section search. Simplifying the procedure even further, one can derive $\Delta_{i}$ as a function of $\theta^{*}$ using first-order optimality conditions, that is, the value of $\Delta_{i}$ which, if the inner minimization were solved, would yield solution $\theta^{*}$. Then, the bisection method over $\theta^{*}$ would select $\theta^{*}$, calculate the corresponding $\Delta_{i}$, and then calculate $\epsilon_{i}^{\text {prio }}$. For bound (GMF1),

$$
\Delta_{i}\left(\theta^{*}\right)=\sum_{k \in K_{i}} \frac{\mu_{i k} \cosh \left(\theta^{*}\right)+\sinh \left(\theta^{*}\right)}{\mu_{i k} \sinh \left(\theta^{*}\right)+\cosh \left(\theta^{*}\right)}
$$

For bound (GMF1'),

$$
\Delta_{i}\left(\theta^{*}\right)=\sqrt{\left|J_{i}\right|} \frac{\bar{\mu}_{i} \cosh \left(\theta^{*} / \sqrt{\left|J_{i}\right|}\right)+\sinh \left(\theta^{*} / \sqrt{\left|J_{i}\right|}\right)}{\mu_{i k} \sinh \left(\theta^{*} / \sqrt{\left|J_{i}\right|}\right)+\cosh \left(\theta^{*} / \sqrt{\left|J_{i}\right|}\right)} .
$$


While there is no upper bound on $\theta^{*}$, it can be observed that relatively low values of $\theta^{*}$ (magnitude of 10 to 100 ) will yield $\Delta_{i}$ values very close to their maximum attainable values due to rapid convergence of $\Delta_{i}\left(\theta^{*}\right)$ to a constant $\left(\left|K_{i}\right|\right.$ and $\sqrt{\left|J_{i}\right|}$ for bounds (GMF1) and $\left(\mathrm{GMF} 1^{\prime}\right)$, respectively). For the a posteriori bound (PKL), which also contains an inner minimization, Proposition 1 can convert solving the inner minimization with optimization to simply meeting first-order optimality conditions with a root-finding method.

\section{Comparisons of a priori bounds}

This section compares the existing and new a priori bounds computationally with two example problems. A summary of their comparisons that is not tied to computational results but is based on their mathematical structure is given in Table 9 .

\subsection{Example MILP}

We first demonstrate the tightness of the proposed a priori bounds through case studies that evaluate $\Delta_{i}$ and the resulting objective function values of an uncertain MILP. Tightness refers to the ability of an a priori bound to produce a smaller $\Delta_{i}$ parameter at the same probability of constraint violation, which can result in a better objective function value. To investigate this, a simple MILP is adapted from Example $7.1 \mathrm{in} \mathrm{Li} \mathrm{et} \mathrm{al.} \mathrm{(2011).} \mathrm{The} \mathrm{nominal} \mathrm{MILP} \mathrm{is}$

$$
\begin{array}{cl}
\max _{x, y} & \widetilde{3} x_{1}+\widetilde{2} x_{2}-\widetilde{10} y_{1}-5 y_{2} \\
\text { s.t. } & \widetilde{1} x_{1}+\widetilde{1} x_{2} \leq \widetilde{20} \\
& \widetilde{1} x_{1}+\widetilde{2} x_{2} \leq \widetilde{12} \\
& x_{1}-20 y_{1} \leq 0 \\
& x_{2}-20 y_{3} \leq 0 \\
& \widetilde{1} x_{1}-\widetilde{1} x_{2} \leq-\widetilde{4} \\
& 0 \leq x_{1} \leq 10 \\
& 0 \leq x_{2} \leq 10 \\
& y_{1}, y_{2} \in\{0,1\} .
\end{array}
$$

For these case studies, uncertainty will be considered in selected right-hand side, left-hand side, and objective function parameters. Specifically, all parameters denoted with a tilde (12 in total) are subject to bounded uncertainty. Each uncertain constraint $i$ has three uncertain parameters $\left(\left|J_{i}\right|=3\right)$. The values of all $\hat{a}_{i j}$ parameters will be $10 \%$ of the nominal parameter values. With equivalent assumptions for each parameter and equivalent uncertainty set types for each constraint, applying the same bound to each uncertain constraint $i$ will yield the same value of $\Delta_{i}$. For example, the resulting robust counterpart when 
applying a box uncertainty set with each uncertain constraint is

$$
\begin{array}{ll}
\max _{x, y, z} & z \\
\text { s.t. } & z-3 x_{1}-2 x_{2}+10 y_{1}+5 y_{2}+\Psi\left(0.3 x_{1}+0.2 x_{2}+y_{1}\right) \leq 0 \\
& x_{1}+x_{2}+\Psi\left(0.1 x_{1}+0.1 x_{2}+2\right) \leq 20 \\
& x_{1}+2 x_{2}+\Psi\left(0.1 x_{1}+0.2 x_{2}+1.2\right) \leq 12 \\
& x_{1}-20 y_{1} \leq 0 \\
& x_{2}-20 y_{3} \leq 0 \\
& x_{1}-x_{2}+\Psi\left(0.1 x_{1}+0.1 x_{2}+0.4\right) \leq-4 \\
& 0 \leq x_{1} \leq 10 \\
& 0 \leq x_{2} \leq 10 \\
& y_{1}, y_{2} \in\{0,1\}
\end{array}
$$

\subsubsection{Bounded probability distributions with known means}

If the assumption can be made that the expected values of all random variables are zero, then four a priori bounds (and two ellipsoidal-specific variants) are immediately relevant, although bounds (GMF1) and (GMF1') simplify to bounds (GMF2) and (GMF2'), respectively. Going beyond this to an assumption of symmetry includes two more bounds, leaving six that are relevant for the case of bounded, symmetric probability distributions. The utility of these bounds are shown in Figures 1 to 6; (GMF1) and (GMF1') are not plotted due to their redundancy with $(\mathrm{GMF} 2)$ and $\left(\mathrm{GMF} 2^{\prime}\right)$, respectively.

Note that the figures are constructed from the perspective of selecting a particular $\Delta_{i}$ that will guarantee a desired $\epsilon_{i}^{\text {prio }}$. For example, bounds (BS2) and (GMF3) cannot return probabilities greater than 0.5; if the probability of constraint violation should be less than or equal to 0.6 , it is optimal to utilize the smallest $\Delta_{i}$ (1 or 0 , respectively) which does not return a probability exceeding 0.6. Bound (GMF3) is upper semi-continuous at positive integer values; it returns the same probability at positive integer $\Delta_{i}$ as well as just below $\Delta_{i}$ at $\Delta_{i}^{-}$, and a different (lower) probability just above $\Delta_{i}$ at $\Delta_{i}^{+}$. For any $\epsilon_{i}^{\text {prio }}$ falling between the probabilities around $\Delta_{i}$, selecting the larger value, $\Delta_{i}^{+}$, validly constrains the probability of constraint violation, while selecting $\Delta_{i}$ does not. Thus, bound (GMF3) is plotted with open circles at $\Delta_{i}^{+}$and the higher probability.

Figures 1 to 6 display the strong performance of (GMF3) for each uncertainty set; despite the discontinuous nature of the bound, it will provide the tightest $\Delta_{i}$ values and best objective function values for any probability of constraint violation in the box, polyhedral, or interval + polyhedral uncertainty sets. This is clearly seen in with the example MILP $\left(\left|J_{i}\right|=3\right)$. With ellipsoidal or interval + ellipsoidal sets, at higher $\left|J_{i}\right|$ values and low probabilities of constraint violation, there are cases in which $\left(\mathrm{GMF}^{\prime}\right)$ provides better results than (GMF3). This is seen in Figure 3 at low probabilities of constraint violation for the example MILP. Thus, this case study demonstrates that when the only assumption regarding the probability distributions is that they are bounded and 


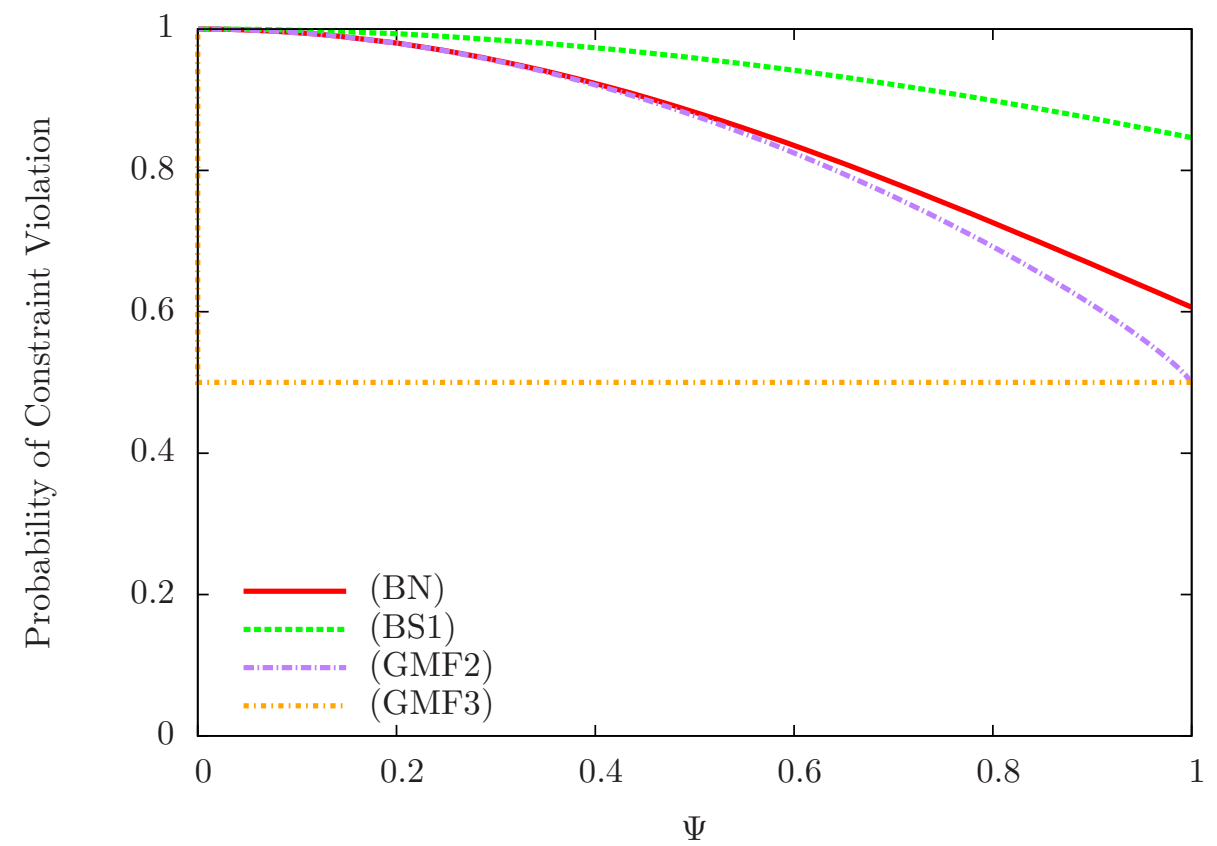

Figure 1: Probability of constraint violation as a function of parameter $\Psi$ for the box uncertainty set using a priori probabilities of constraint violation for unspecified but bounded and symmetric probability distributions, $\left|J_{i}\right|=3$. 


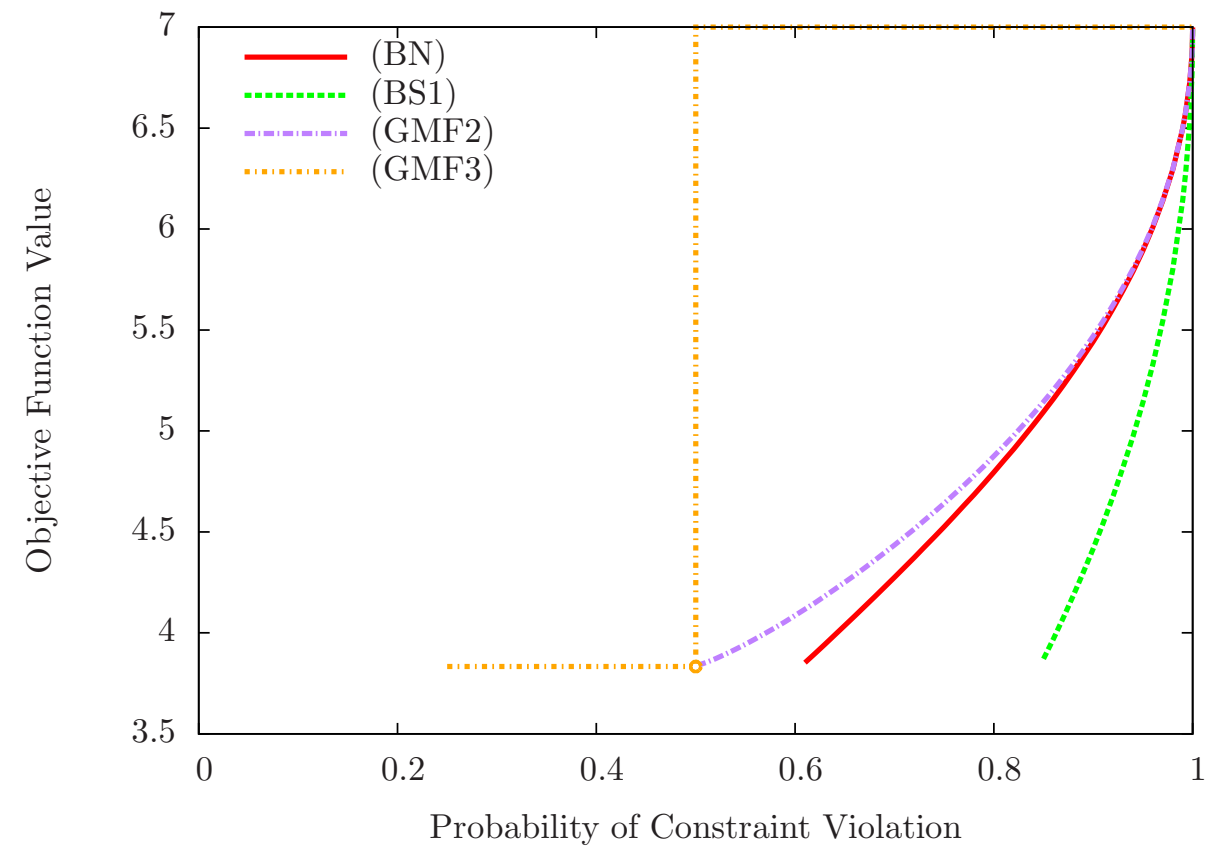

Figure 2: Objective value from the example MILP at varying probabilities of constraint violation for the box uncertainty set, using a priori bounds for unspecified but bounded and symmetric probability distributions, $\left|J_{i}\right|=3$. 


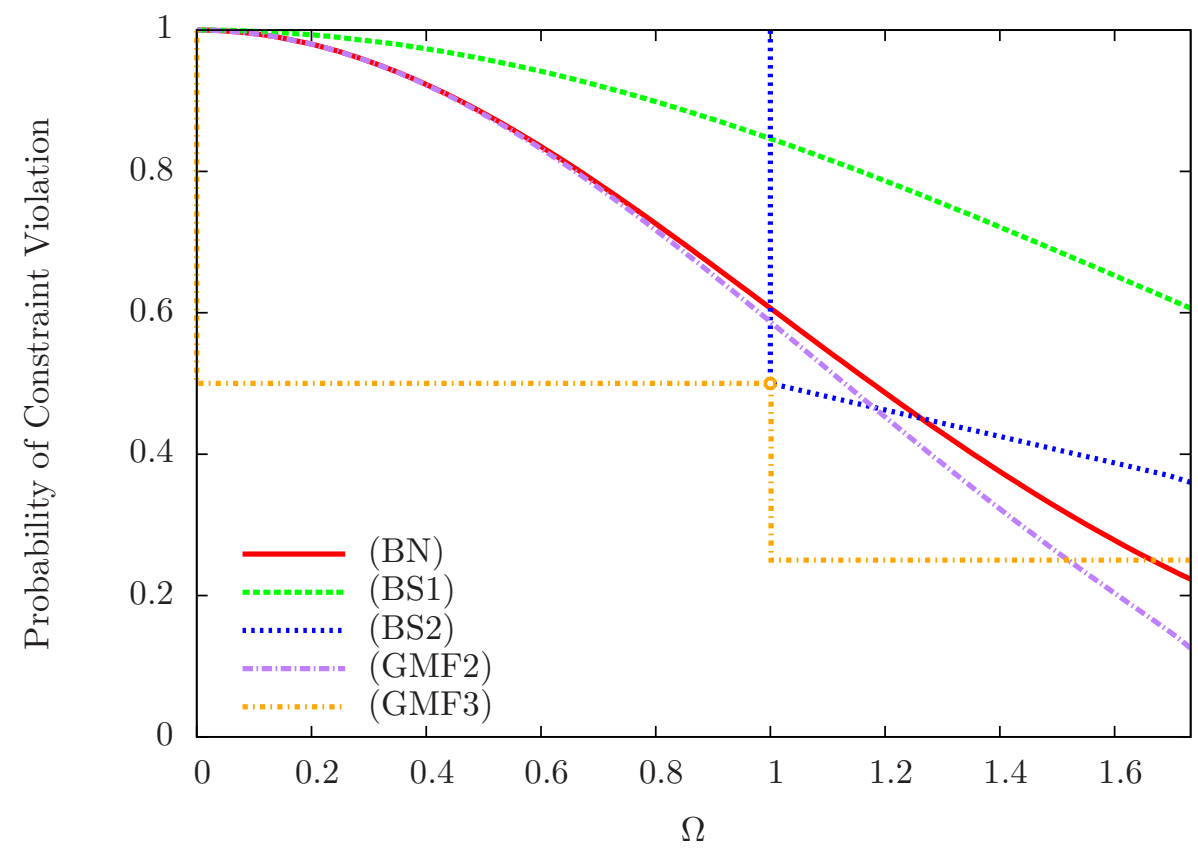

Figure 3: Probability of constraint violation as a function of parameter $\Omega$ for the interval + ellipsoidal uncertainty set using a priori probabilities of constraint violation for unspecified but bounded and symmetric probability distributions, $\left|J_{i}\right|=3$. 


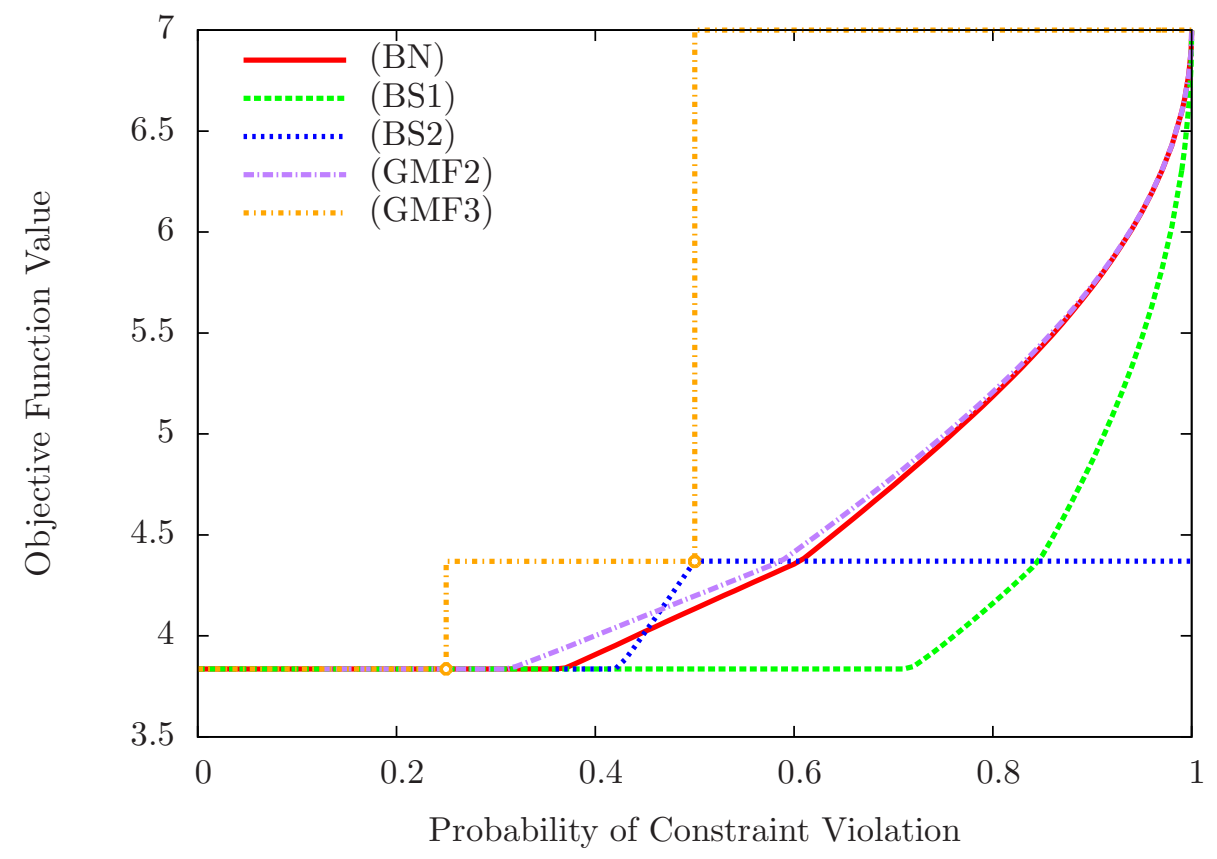

Figure 4: Objective value from the example MILP at varying probabilities of constraint violation for the interval + ellipsoidal uncertainty set, using a priori bounds for unspecified but bounded and symmetric probability distributions, $\left|J_{i}\right|=3$. 


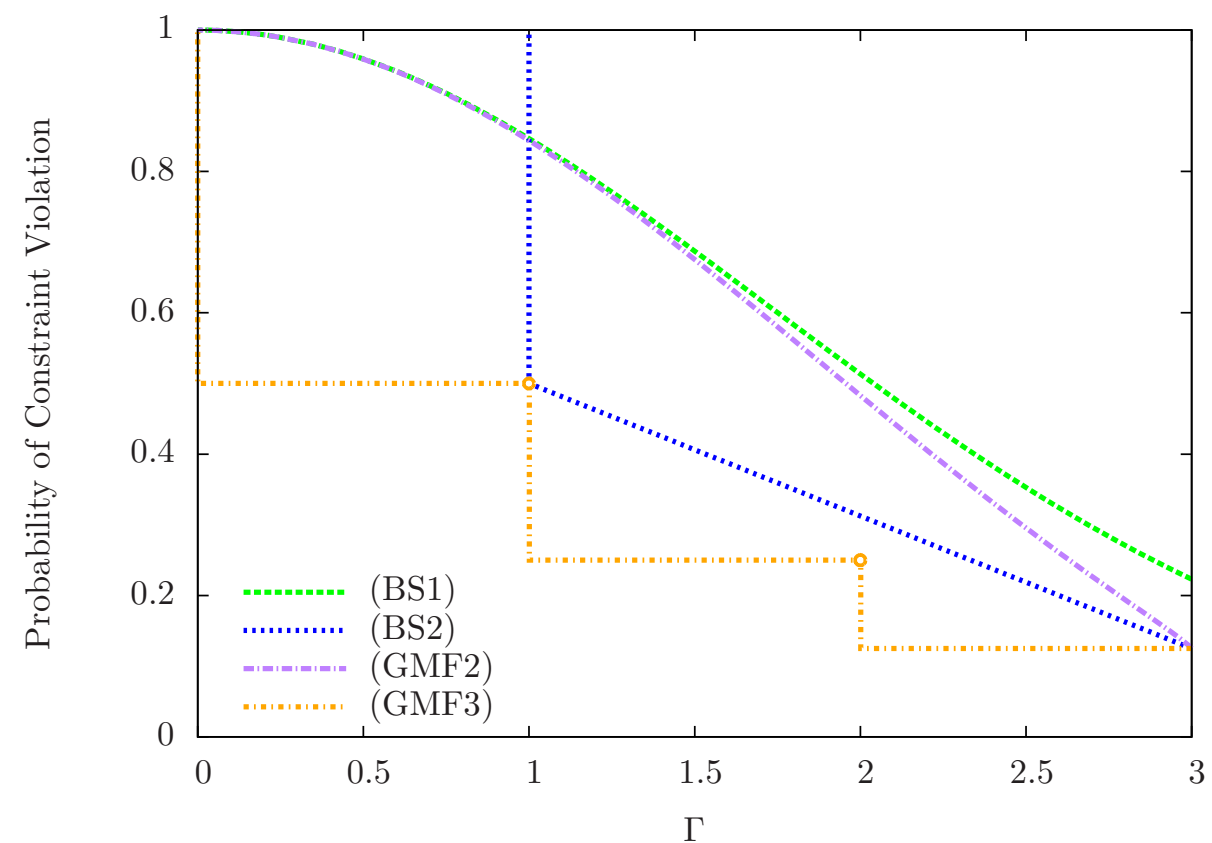

Figure 5: Probability of constraint violation as a function of parameter $\Gamma$ for the interval + polyhedral uncertainty set using a priori probabilities of constraint violation for unspecified but bounded and symmetric probability distributions, $\left|J_{i}\right|=3$. 


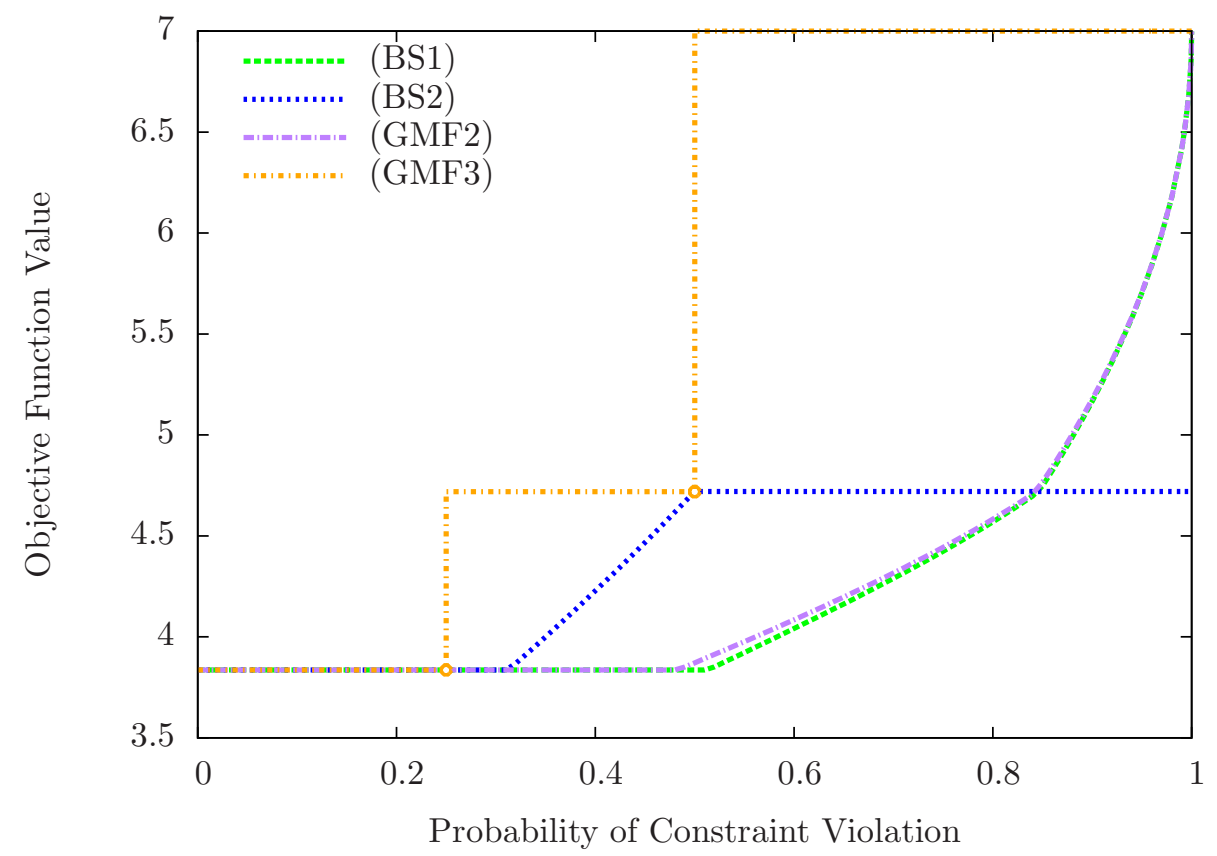

Figure 6: Objective value from the example MILP at varying probabilities of constraint violation for the interval + polyhedral uncertainty set, using a priori bounds for unspecified but bounded and symmetric probability distributions, $\left|J_{i}\right|=3$. 


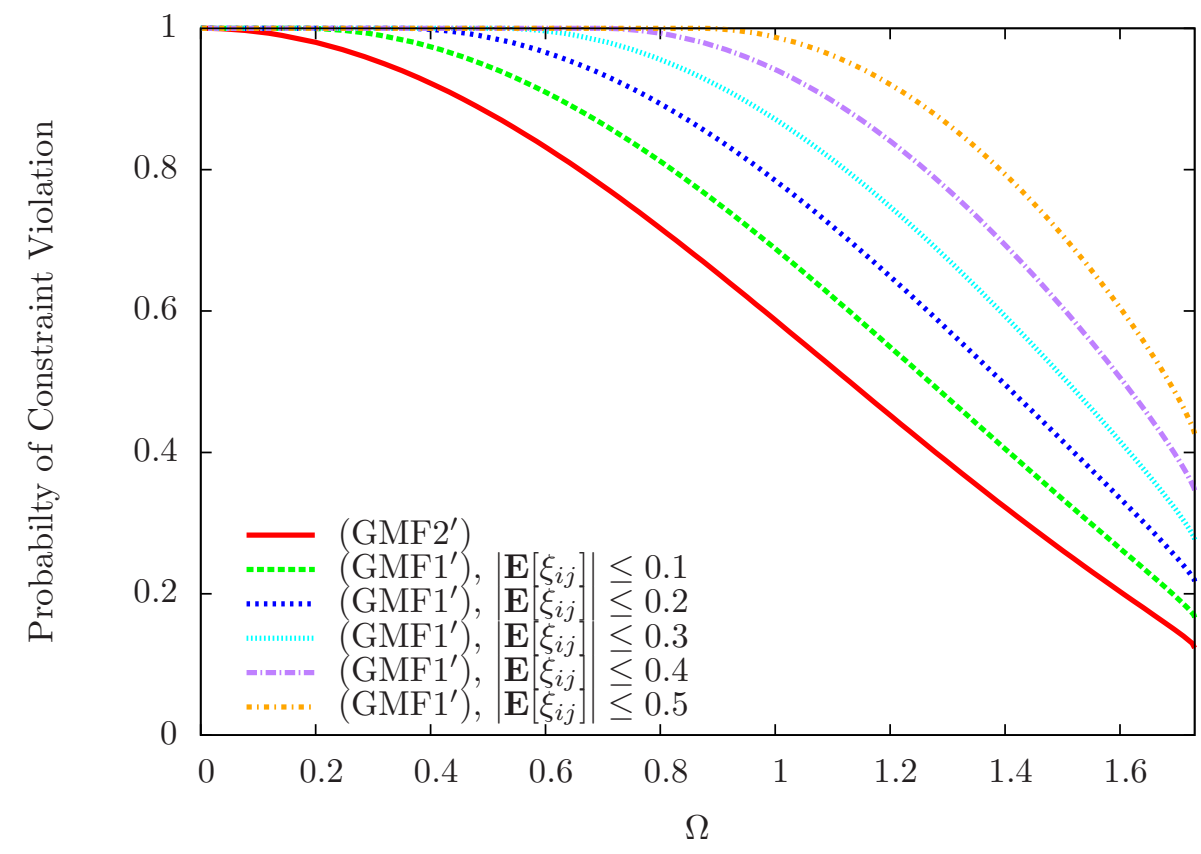

Figure 7: Probability of constraint violation as a function of $\Omega$ for $\left(\mathrm{GMF} 1^{\prime}\right)$ on an interval + ellipsoidal uncertainty set with increasingly loose bounds on $E\left[\xi_{i j}\right],\left|J_{i}\right|=3$.

symmetric, bounds (GMF3) and (GMF2') are the only two which should be considered.

\subsubsection{Bounded probability distributions with bounded means}

Bounds (GMF1) and (GMF1') permit robust counterpart optimization even in the case where the mean of the distribution of an uncertain parameter is not known exactly, but is assumed to lie within a range of values. The performances of (GMF1) and (GMF1') with the interval + polyhedral and interval + ellipsoidal sets, respectively, were evaluated with $\left|J_{i}\right|=3$ and $\left|J_{i}\right|=12$. The expected value of every uncertain parameter is not known exactly; a range of values is specified instead. The bounds on $\left|\mathbf{E}\left[\xi_{i j}\right]\right|$, denoted as $\mu_{i j}$, were set at equal values, $\forall j \in J_{i}$, ranging from 0.1 to 0.5 with increments of 0.1 . The results can be seen in Figures 7 through 10. Despite the fact that the only given information on the uncertain parameters is that (i) they are bounded, and (ii) there is an interval that their expected values lie within, bounds (GMF1) and $\left(\mathrm{GMF}^{\prime}\right)$ provide tight results which are comparable to the bounds that assume $\mathbf{E}\left[\xi_{i j}\right]=0$. At $\left|J_{i}\right|=12$ and $\mu_{i j}=0.5$, that is, with the true expected value of a parameter existing anywhere across $50 \%$ of the total range of the parameter support, better than worst-case values of $\Delta_{i}$ are able to be obtained at, say, $\epsilon_{i}^{\text {prio }}=0.05$ with the interval + ellipsoidal or interval + polyhedral set. 


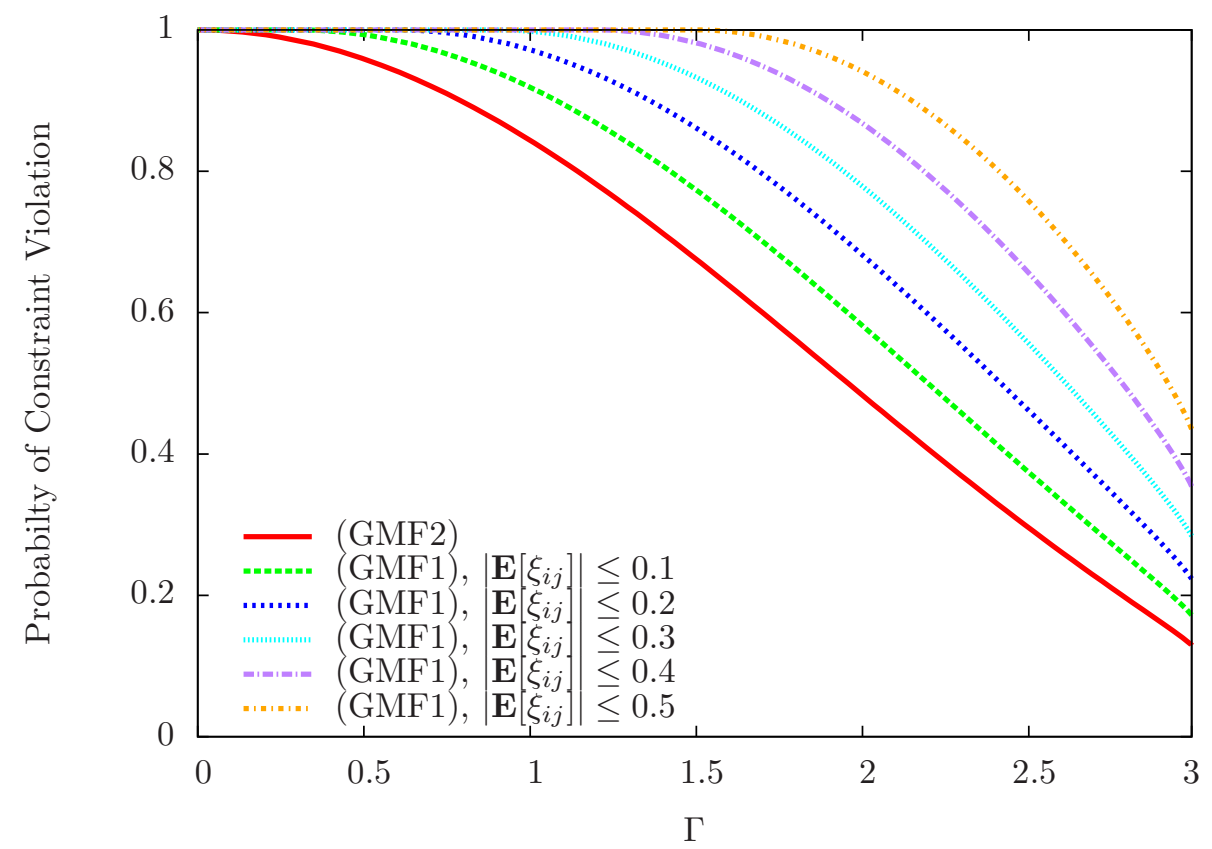

Figure 8: Probability of constraint violation as a function of $\Gamma$ for (GMF1) on an interval + polyhedral uncertainty set with increasingly loose bounds on $E\left[\xi_{i j}\right],\left|J_{i}\right|=3$. 


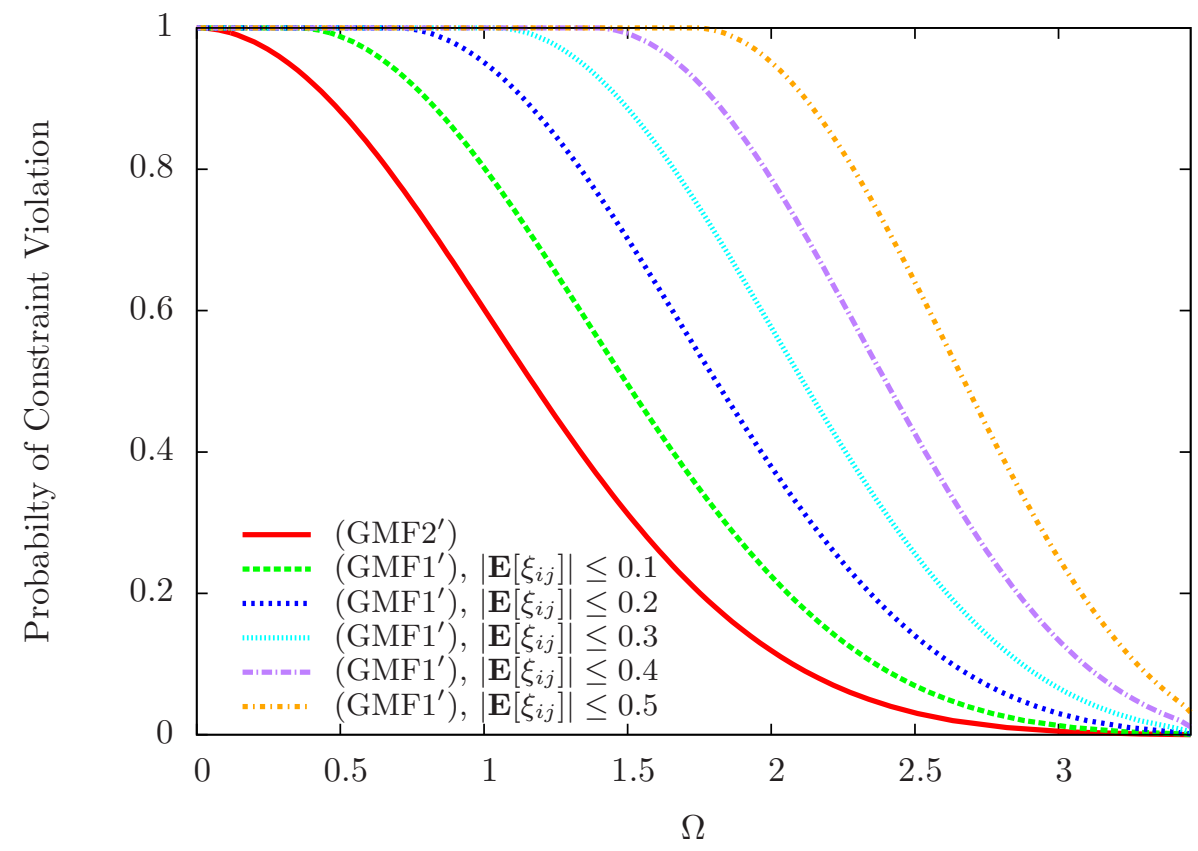

Figure 9: Probability of constraint violation as a function of $\Omega$ for $\left(\mathrm{GMF} 1^{\prime}\right)$ on an interval + ellipsoidal uncertainty set with increasingly loose bounds on $E\left[\xi_{i j}\right],\left|J_{i}\right|=12$. 


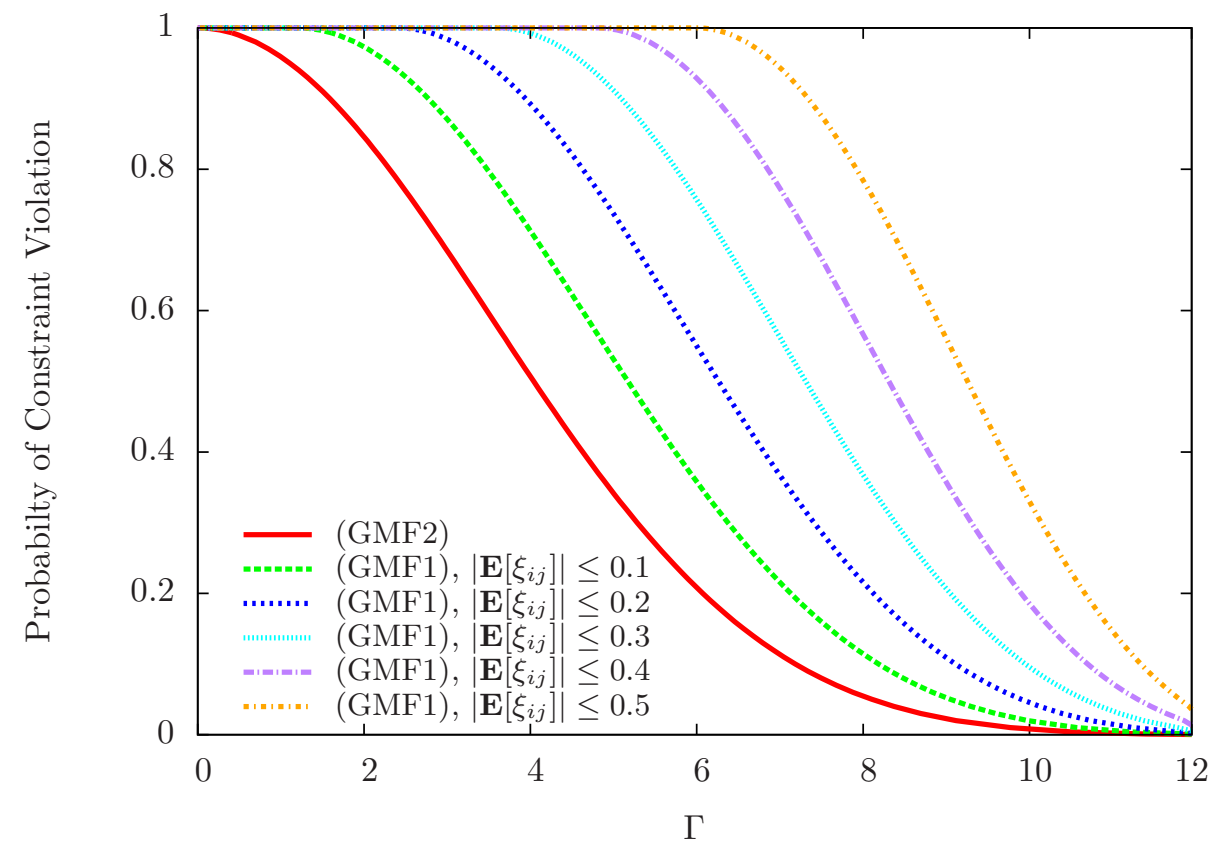

Figure 10: Probability of constraint violation as a function of $\Gamma$ for (GMF1) on an interval + polyhedral uncertainty set with increasingly loose bounds on $E\left[\xi_{i j}\right],\left|J_{i}\right|=12$. 


\subsection{Application: Multiperiod Production Planning LP}

A linear programming application of robust optimization exists in multiperiod production planning. An example LP model is expanded from (Ravindran, 2008) and (Li et al., 2012) in which a company is planning for an upcoming year. The profit for the company is to be maximized over the year, which is broken into 12 months each with unique parameters relating to production. The company also must operate within a given annual budget, in this case, $\$ 800,000$. The model is written to decide how much product should be produced each month, given fluctuating production costs, selling prices, storage costs, production capacity, and product demand. Operations begin in period one, with the company beginning with 500 tons of product in storage; at the end of the year, the company seeks to have this same amount of product in storage again. The product produced in each period can be sold immediately, or stored until being sold in a future period. This problem is formulated as the following linear optimization problem:

$$
\begin{array}{lll}
\max _{x, y, z} & \sum_{j}\left(P_{j} z_{j}-C_{j} x_{j}-V_{j} y_{j}\right) & \\
\text { s.t. } & \sum_{j}\left(C_{j} x_{j}+V_{j} y_{j}\right) \leq B & \\
& & \\
& I+x_{1}-\left(y_{1}+z_{1}\right)=0 & \\
& y_{j-1}+x_{j}-\left(y_{j}+z_{j}\right)=0 & \forall j=2, \ldots, 12 \\
& y_{12}=F & \\
& x_{j} \leq U_{j} & \forall j \\
& z_{j} \leq D_{j} & \forall j \\
& x_{j}, y_{j}, z_{j} \geq 0 & \forall j .
\end{array}
$$

In this formulation, $x_{j}, y_{j}$, and $z_{j}$ are variables representing the production amount, amount of product in storage, and amount of product sold (tons) in each period, respectively. Parameters $P_{j}, C_{j}$, and $V_{j}$ are financial parameters representing the selling price, production cost, and storage cost per ton of product. The amount sold in each period is limited by demand $D_{j}$, and the amount produced is limited by the production capacity $U_{j}$. Finally, the overall budget $B=\$ 800,000$ limits the total cost of production, while $I=F=500$ tons, representing the initial $(I)$ and final $(F)$ amount of product in storage. The nominal values for each parameter in each month are shown in Table 1.

To analyze the new a priori bounds on the probability of constraint violation, uncertainty is considered in the selling price of the product. Thus, uncertainty is seen in the objective function alone and $\left|J_{i}\right|=12$. The objective function can be transformed in the following manner such that variable $\zeta$ is maximized with the added constraint

$$
\zeta-\sum_{j} \tilde{P}_{j} z_{j}+\sum_{j}\left(C_{j} x_{j}+V_{j} y_{j}\right) \leq 0 .
$$




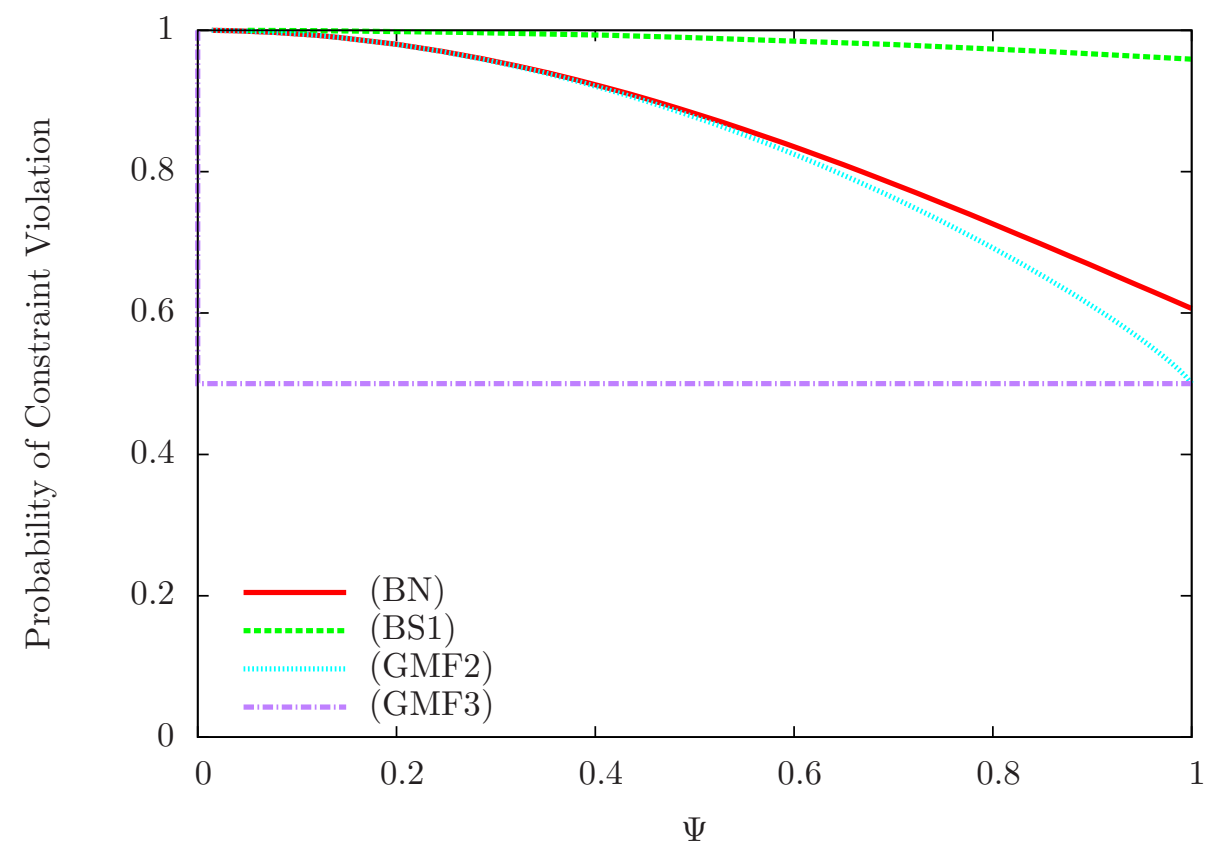

Figure 11: Probability of constraint violation from $\Psi$ values for the box uncertainty set considering uncertainty in the objective function of the multiperiod planning problem, $\left|J_{i}\right|=12$. A uniform distribution is assumed for all uncertain parameters.

Then, the robust counterpart can be formulated, where $\tilde{P}_{j}$ represents the actual selling price and is described by $\tilde{P}_{j}=P_{j}+\xi_{j} \hat{P}_{j}$. It is assumed that the perturbations $\hat{P}$ are at most $20 \%$ of the nominal values $P_{j}$. Bounds presented in this paper are then applied to the box, interval + ellipsoidal, and interval + polyhedral uncertainty sets. The results are shown in Figures 11 through 16.

The new a priori probabilistic bounds are quantified for six different probabilities of constraint violation ranging from $5 \%$ to $30 \%$. Traditionally, a "price of robustness" (Bertsimas and Sim, 2004) is used as a metric for conservatism by evaluating the change in the objective function relative to the nominal value when solving the robust counterpart formulation. If $\zeta_{N}$ and $\zeta_{R}$ represent the nominal and robust objective function values, respectively, then the price of robustness $(\mathrm{PoR})$ is calculated as

$$
\mathrm{PoR}=\left|\frac{\zeta_{N}-\zeta_{R}}{\zeta_{N}}\right|
$$

A new quantification is proposed for bounded uncertainty to represent conservatism in a way that is not as dependent on the nominal objective function value. This "normalized price of robustness" (NPoR) is defined using the gap 


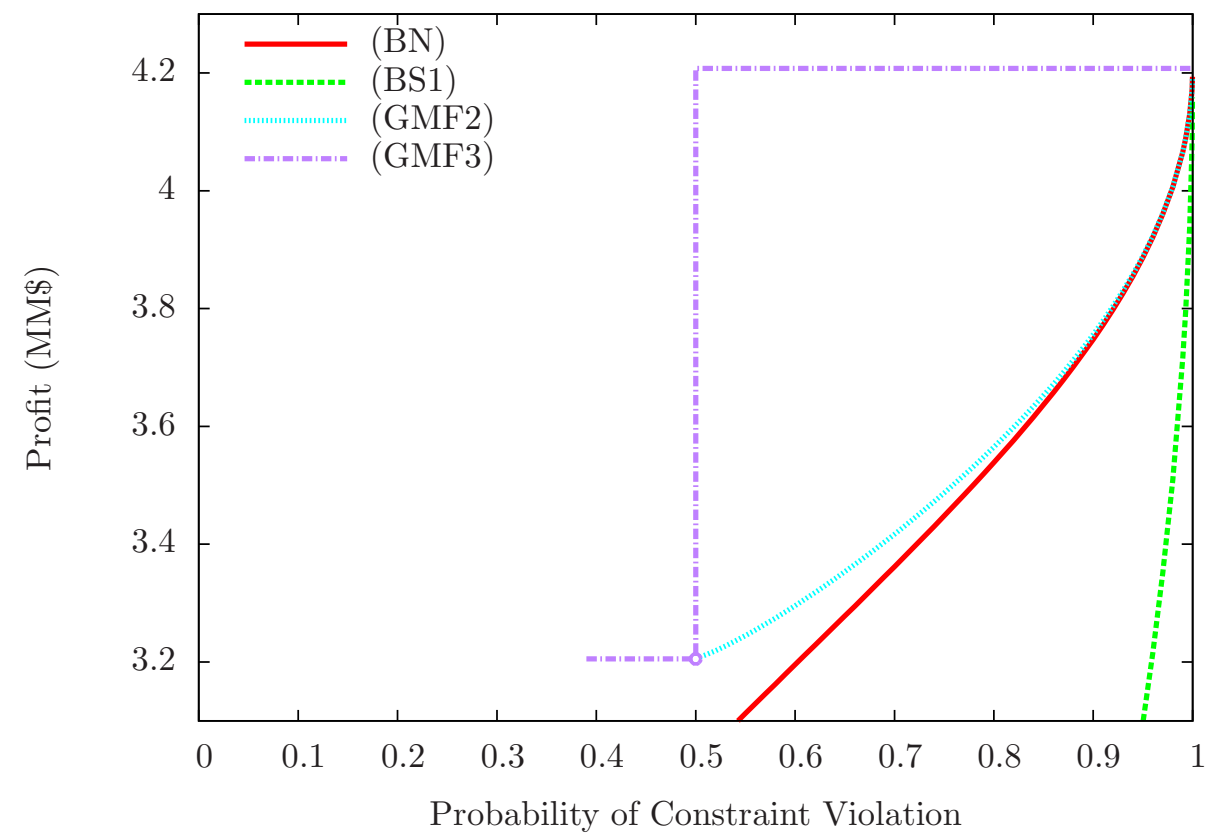

Figure 12: Profit from the multiperiod planning problem as a function of probability of constraint violation for the box uncertainty set with parameters described by a uniform distribution, $\left|J_{i}\right|=12$. 


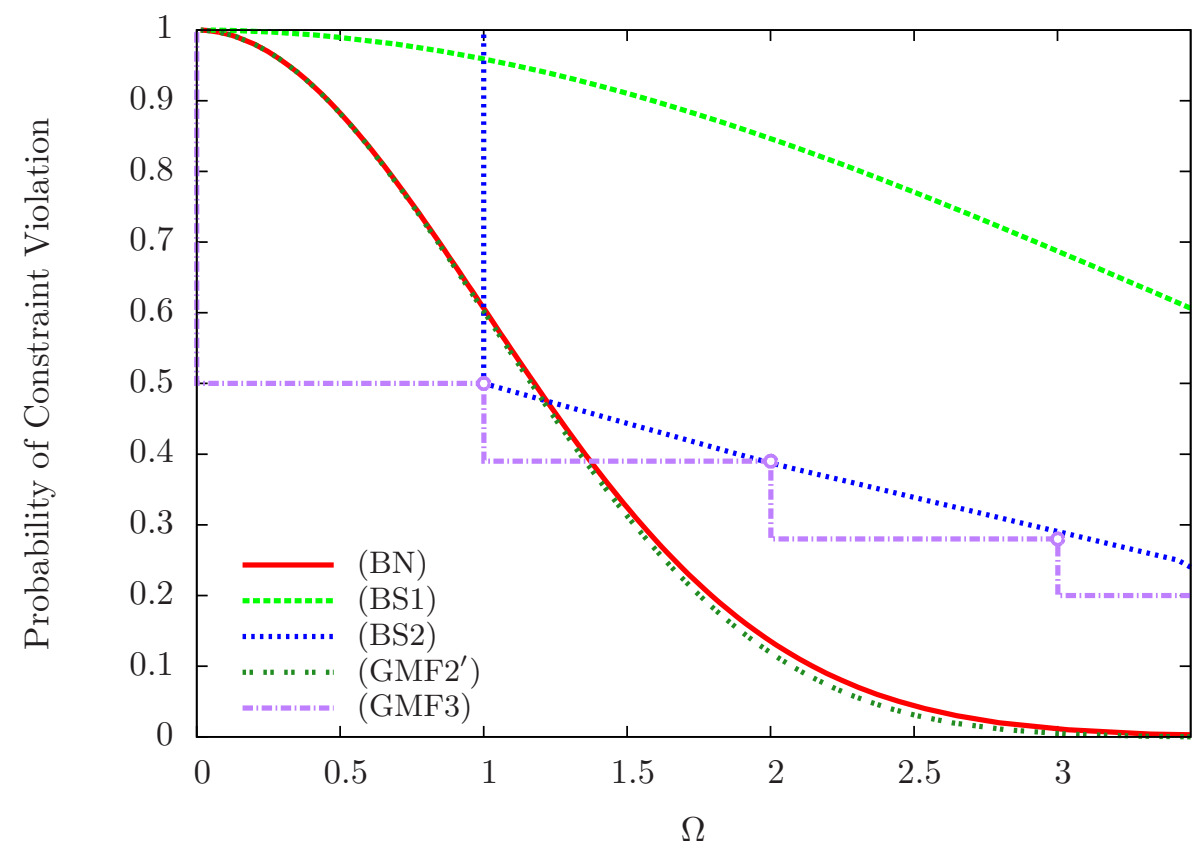

Figure 13: Probability of constraint violation from $\Omega$ values for the interval + ellipsoidal uncertainty set considering uncertainty in the objective function of the multiperiod planning problem, $\left|J_{i}\right|=12$. A uniform distribution is assumed for all uncertain parameters. 


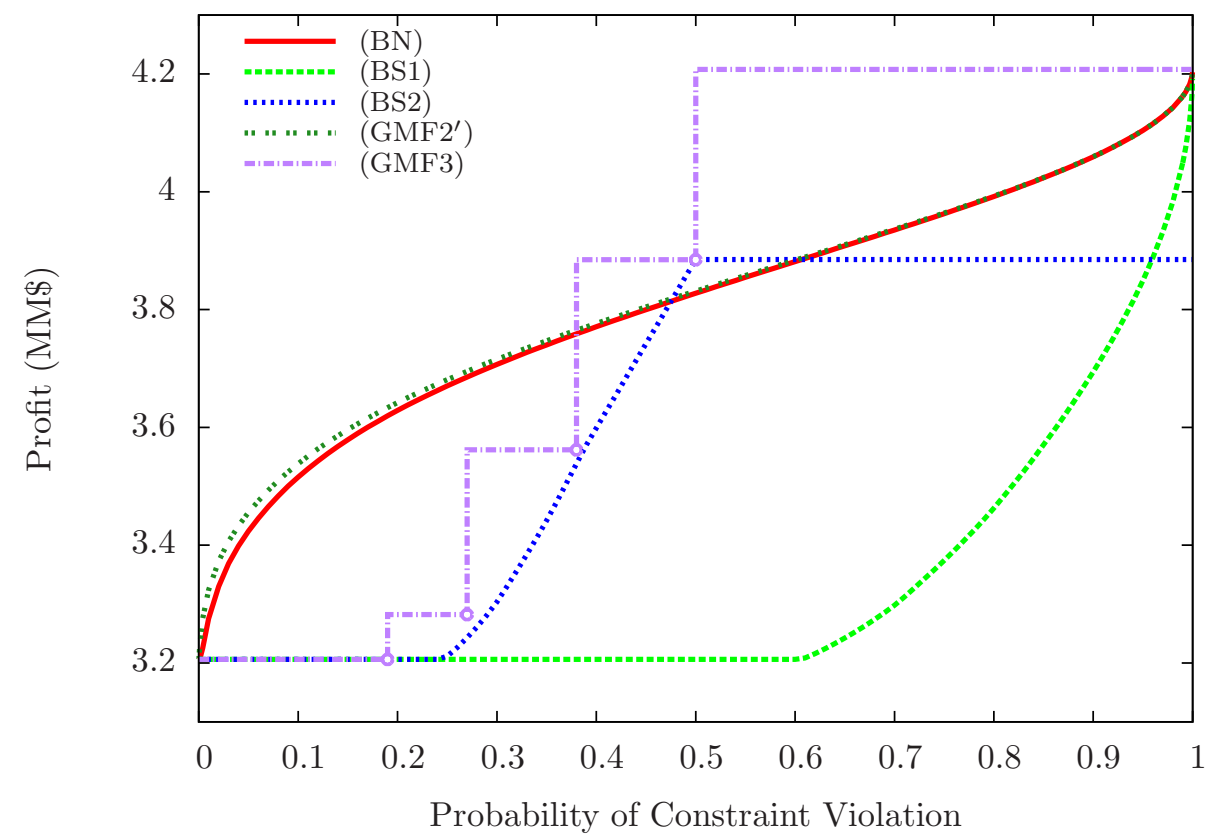

Figure 14: Profit from the multiperiod planning problem as a function of probability of constraint violation for the interval + ellipsoidal uncertainty set with parameters described by a uniform distribution, $\left|J_{i}\right|=12$. 


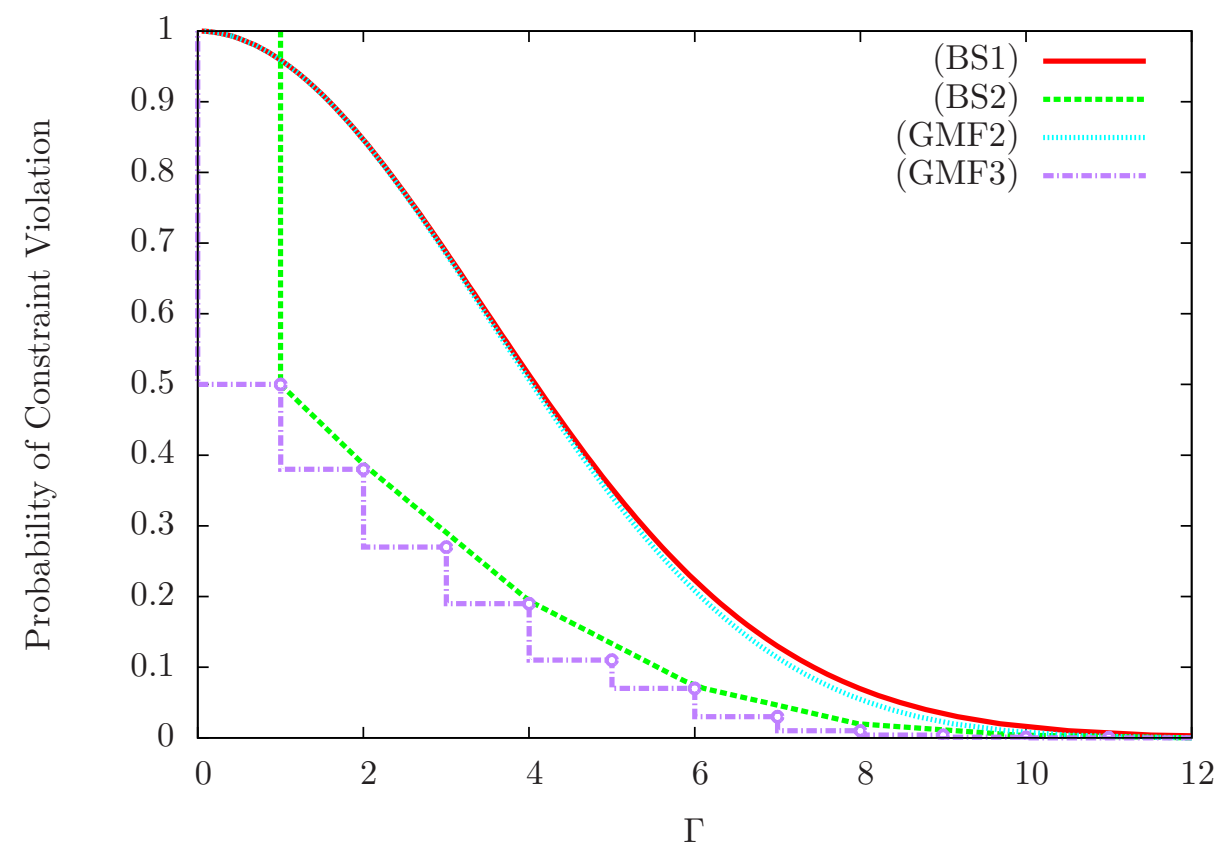

Figure 15: Probability of constraint violation from $\Gamma$ values for the interval + polyhedral uncertainty set considering uncertainty in the objective function of the multiperiod planning problem, $\left|J_{i}\right|=12$. A uniform distribution is assumed for all uncertain parameters. 


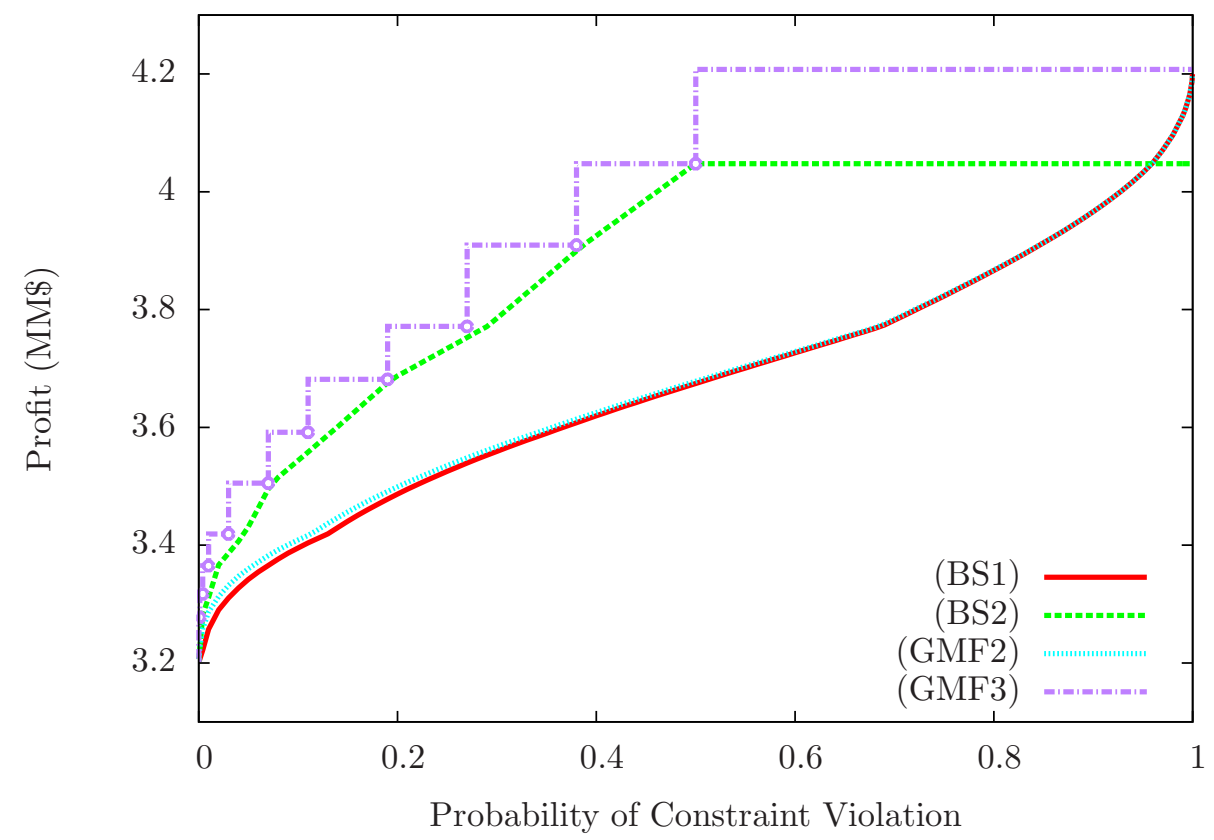

Figure 16: Profit from the multiperiod planning problem as a function of probability of constraint violation for the interval + polyhedral uncertainty set with parameters described by a uniform distribution, $\left|J_{i}\right|=12$. 
between the nominal and worst case $\left(\zeta_{W C}\right)$ objective function values such that

$$
\mathrm{NPoR}=\left|\frac{\zeta_{N}-\zeta_{R}}{\zeta_{N}-\zeta_{W C}}\right| .
$$

The worst case value can simply be calculated using a box uncertainty set with $\Psi=1$, and NPoR will vary from values of 0 at the nominal case to 1 at the worst case.

The inclusion of uncertainty is most notable in the reduction of profit from $\$ 4,207,705.88$ at the nominal case to $\$ 3,206,164.71$ in the worst case scenario, as seen in all of the objective function figures. Yet, the conservatism of the worst case values can be drastically reduced, as seen Figures 14 and 16, where even a $1 \%$ probability of constraint violation using (GMF3) leads to a $\$ 159,000$ increase $(5 \%$ increase) in profit compared to the worst case for the interval + polyhedral set. Bound (GMF3) performs the strongest for all probabilities of constraint violation for the interval + polyhedral set, and is dominant at high probabilities of constraint violation for the interval + ellipsoidal set. For the interval + ellipsoidal case, the new (GMF2') outperforms the existing (BN).

These results are reiterated through Tables 2 and 3 for the interval + ellipsoidal and interval + polyhedral sets, respectively. Bounds $(\mathrm{BN})$ and $\left(\mathrm{GMF}^{\prime}\right)$ are the only bounds to reach an NPoR of $50 \%$ or less at $\epsilon_{i}^{\text {prio }}=0.3$ and 0.35 . Bound (GMF2') displays the best performance of all bounds at low probabilities of constraint violation, with the biggest improvements over (BN) coming at the lowest probabilities of constraint violation. For the interval + polyhedral case, (GMF3) is the strongest performer; at only $20 \%$ probability of constraint violation, this bound leads to profits closer to the nominal than the worst case. At $30 \%$ probability of constraint violation, the profit has been reduced by only $7 \%$ due to the inclusion of uncertainty. These results demonstrate that for unknown distributions with these $\left|J_{i}\right|$ values, the a priori bounds are tighter for the interval + polyhedral set than the interval + ellipsoidal set. 
Table 1: Monthly Planning Data for the LP Production Planning Problem

\begin{tabular}{cccccc}
\hline Period & $\begin{array}{c}\text { Selling Price } \\
(\$ / \text { ton })\end{array}$ & $\begin{array}{c}\text { Production Cost } \\
(\$ / \text { ton })\end{array}$ & $\begin{array}{c}\text { Storage Cost } \\
(\$ / \text { ton })\end{array}$ & $\begin{array}{c}\text { Production Capacity } \\
(\text { tons })\end{array}$ & $\begin{array}{c}\text { Demand } \\
(\text { tons })\end{array}$ \\
$\mathrm{j}$ & $P_{j}$ & $C_{j}$ & $V_{j}$ & $U_{j}$ & $D_{j}$ \\
\hline 1 & 180 & 20 & 2 & 1500 & 1100 \\
2 & 180 & 25 & 2 & 2000 & 1500 \\
3 & 250 & 30 & 2 & 2200 & 1800 \\
4 & 270 & 40 & 2 & 3000 & 1600 \\
5 & 300 & 50 & 2 & 2700 & 2300 \\
6 & 320 & 60 & 2 & 2500 & 2500 \\
7 & 300 & 80 & 2 & 1900 & 2300 \\
8 & 270 & 55 & 2 & 1500 & 1600 \\
9 & 250 & 45 & 2 & 1300 & 1800 \\
10 & 180 & 30 & 2 & 1100 & 1500 \\
11 & 180 & 30 & 2 & 1200 & 1100 \\
12 & 160 & 25 & 2 & 1400 & 1200 \\
\hline
\end{tabular}


Table 2: Comparison of existing and new a priori probabilistic bounds for the multiperiod production planning problem with unknown parameter distributions and an interval + ellipsoidal uncertainty set.

\begin{tabular}{|c|c|c|c|c|c|c|c|c|c|}
\hline \multirow[b]{2}{*}{$\epsilon_{i}^{\text {prio }}$} & \multicolumn{3}{|c|}{$(\mathrm{BN})$} & \multicolumn{3}{|c|}{$\begin{array}{c}\text { Existing Bounds } \\
(\mathrm{BS} 1)\end{array}$} & \multicolumn{3}{|c|}{ (BS2) } \\
\hline & $\begin{array}{l}\text { Profit } \\
(\mathrm{MM} \$)\end{array}$ & $\begin{array}{l}\text { PoR } \\
(\%)\end{array}$ & $\begin{array}{l}\text { NPoR } \\
(\%)\end{array}$ & $\begin{array}{l}\text { Profit } \\
(\mathrm{MM} \$)\end{array}$ & $\begin{array}{l}\text { PoR } \\
(\%)\end{array}$ & $\begin{array}{c}\text { NPoR } \\
(\%)\end{array}$ & $\begin{array}{l}\text { Profit } \\
(\mathrm{MM} \$)\end{array}$ & $\begin{array}{c}\text { PoR } \\
(\%)\end{array}$ & $\begin{array}{c}\text { NPoR } \\
(\%)\end{array}$ \\
\hline 0.05 & 3.424 & 18.63 & 78.27 & 3.206 & 23.80 & 100.00 & 3.206 & 23.80 & 100.00 \\
\hline 0.10 & 3.516 & 16.45 & 69.11 & 3.206 & 23.80 & 100.00 & 3.206 & 23.80 & 100.00 \\
\hline 0.15 & 3.579 & 14.94 & 62.77 & 3.206 & 23.80 & 100.00 & 3.206 & 23.80 & 100.00 \\
\hline 0.20 & 3.629 & 13.76 & 57.81 & 3.206 & 23.80 & 100.00 & 3.206 & 23.80 & 100.00 \\
\hline 0.25 & 3.670 & 12.77 & 53.66 & 3.206 & 23.80 & 100.00 & 3.212 & 23.67 & 99.46 \\
\hline 0.30 & 3.707 & 11.90 & 50.00 & 3.206 & 23.80 & 100.00 & 3.304 & 21.48 & 90.26 \\
\hline \multirow[t]{2}{*}{0.35} & 3.740 & 11.11 & 46.69 & 3.206 & 23.80 & 100.00 & 3.442 & 18.20 & 76.46 \\
\hline & \multicolumn{6}{|c|}{$\begin{array}{c}\text { New Bounds } \\
\text { (GMF3) }\end{array}$} & & & \\
\hline$\epsilon_{i}^{\text {prio }}$ & $\begin{array}{l}\text { Profit } \\
(\mathrm{MM} \$)\end{array}$ & $\begin{array}{l}\text { PoR } \\
(\%)\end{array}$ & $\begin{array}{l}\text { NPoR } \\
(\%)\end{array}$ & $\begin{array}{l}\text { Profit } \\
(\mathrm{MM} \$)\end{array}$ & $\begin{array}{l}\text { PoR } \\
(\%)\end{array}$ & $\begin{array}{l}\text { NPoR } \\
(\%)\end{array}$ & & & \\
\hline 0.05 & 3.455 & 17.88 & 75.12 & 3.206 & 23.80 & 100.00 & & & \\
\hline 0.10 & 3.538 & 15.91 & 66.83 & 3.206 & 23.80 & 100.00 & & & \\
\hline 0.15 & 3.596 & 14.53 & 61.05 & 3.206 & 23.80 & 100.00 & & & \\
\hline 0.20 & 3.642 & 13.44 & 56.48 & 3.282 & 22.00 & 92.42 & & & \\
\hline 0.25 & 3.681 & 12.52 & 52.59 & 3.282 & 22.00 & 92.42 & & & \\
\hline 0.30 & 3.715 & 11.70 & 49.15 & 3.562 & 15.35 & 64.48 & & & \\
\hline 0.35 & 3.747 & 10.95 & 46.00 & 3.562 & 15.35 & 64.48 & & & \\
\hline
\end{tabular}




\section{Comparisons of a posteriori bounds}

We can compare the strength of the a posteriori bounds by applying them to specific solutions $x^{*}$. As in Section 7.2, we solved the multiperiod planning problem at different probabilities of constraint violation using the interval + ellipsoidal and interval + polyhedral uncertainty sets with $\Delta$ specified by a priori bounds (GMF2) and (GMF2'), respectively. A comparison of the probabilities obtained by each a posteriori bound versus the a priori probabilities can be seen in Figures 17 and 19. Any a posteriori bound will provide probabilities that are as tight as or tighter than those provided by its a priori analogue. Note that the a posteriori analogue of bound (GMF2) and (GMF2') is bound (PKL); the probabilities provided by bound $\left(\mathrm{GMF}^{\prime}\right)$ were remarkably close and often equivalent to those provided by bound (PKL), despite the fact that it accounts for all solutions rather than a particular solution. Bound (GMF5) displayed good performance, particularly for the interval + polyhedral set, but could provide worse probabilities than other bounds at times due the fact that it does not similarly account for particular elements of the robust solution. The objective function values corresponding to the a posteriori probabilities can be seen in Figures 18 and 20, with the a priori probabilities and objective function values of bound (GMF2') and (GMF2), respectively, included for comparison. In this example, a priori bound (GMF2') essentially provided a posteriori levels of profit.

\section{Conclusions}

The performance of robust counterpart optimization is reliant upon the relationship between the probabilistic guarantees required by the user and the uncertain parameters included in a robust counterpart. We derived new a priori bounds on the probability of constraint violation which improve upon existing methods and are applicable to models under uncertainty with bounded uncertain parameters and known means, but unspecified probability distributions. We also provided the first a priori and a posteriori bounds for uncertain models with limited information on the means of its uncertain parameters. Given uncertain parameters subject to bounded uncertainty, the new a priori bounds will provide the best objective function values to a robust counterpart at an equivalent probability of constraint violation when compared to existing bounds. If, however, any further knowledge on the distributions of uncertain parameters exists, it can be leveraged to provide even starker improvements to the performance of robust counterpart optimization. We will explore this concept in subsequent work. 


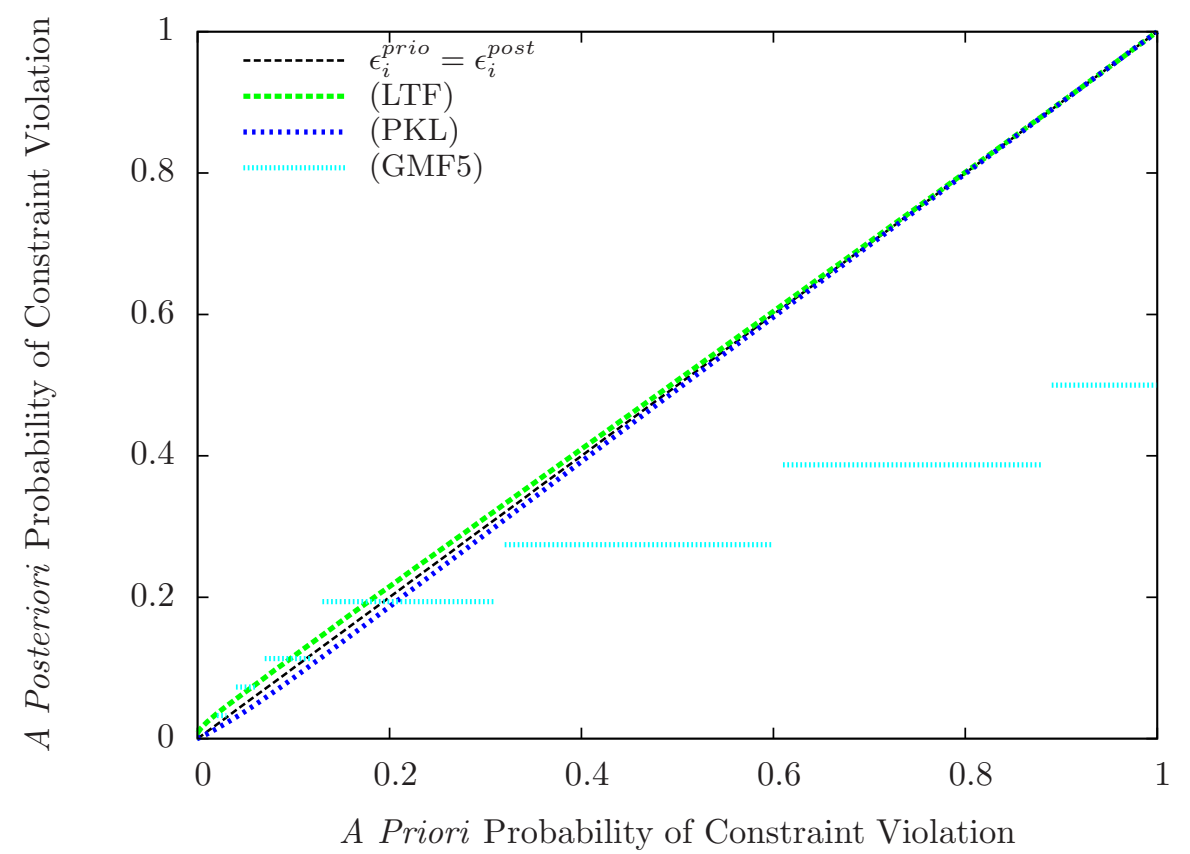

Figure 17: A posteriori probability of constraint violation of each solution obtained from the multiperiod planning problem with the interval + ellipsoidal uncertainty set versus a priori probability distribution; $\epsilon_{i}^{\text {prio }}$ was set by selecting $\Delta_{i}$ with bound (GMF2'). 


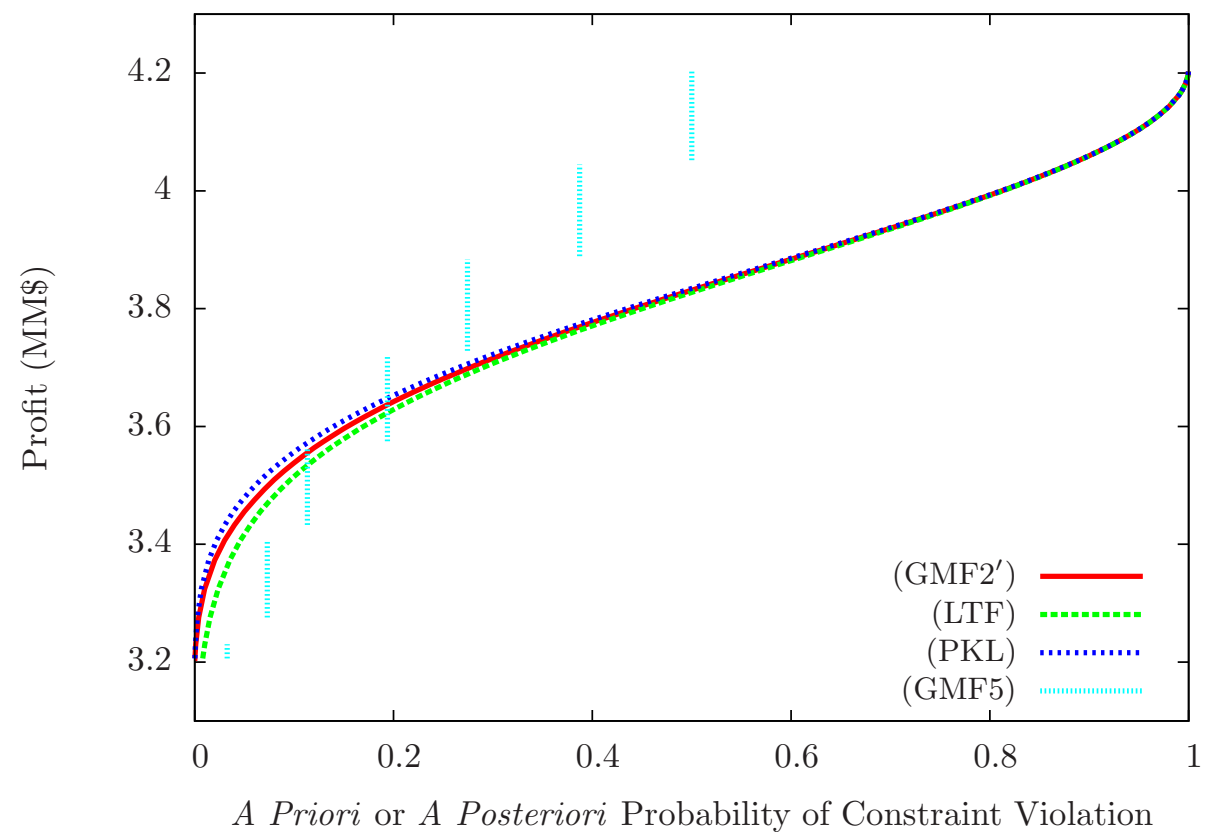

Figure 18: Profit versus a posteriori probability of constraint violation of each solution obtained from the multiperiod planning problem with the interval + ellipsoidal uncertainty set and bound $\left(\mathrm{GMF}^{\prime}\right)$; profit versus $\epsilon_{i}^{\text {prio }}$ as obtained by bound $\left(\mathrm{GMF}^{\prime}\right)$ included for comparison. 


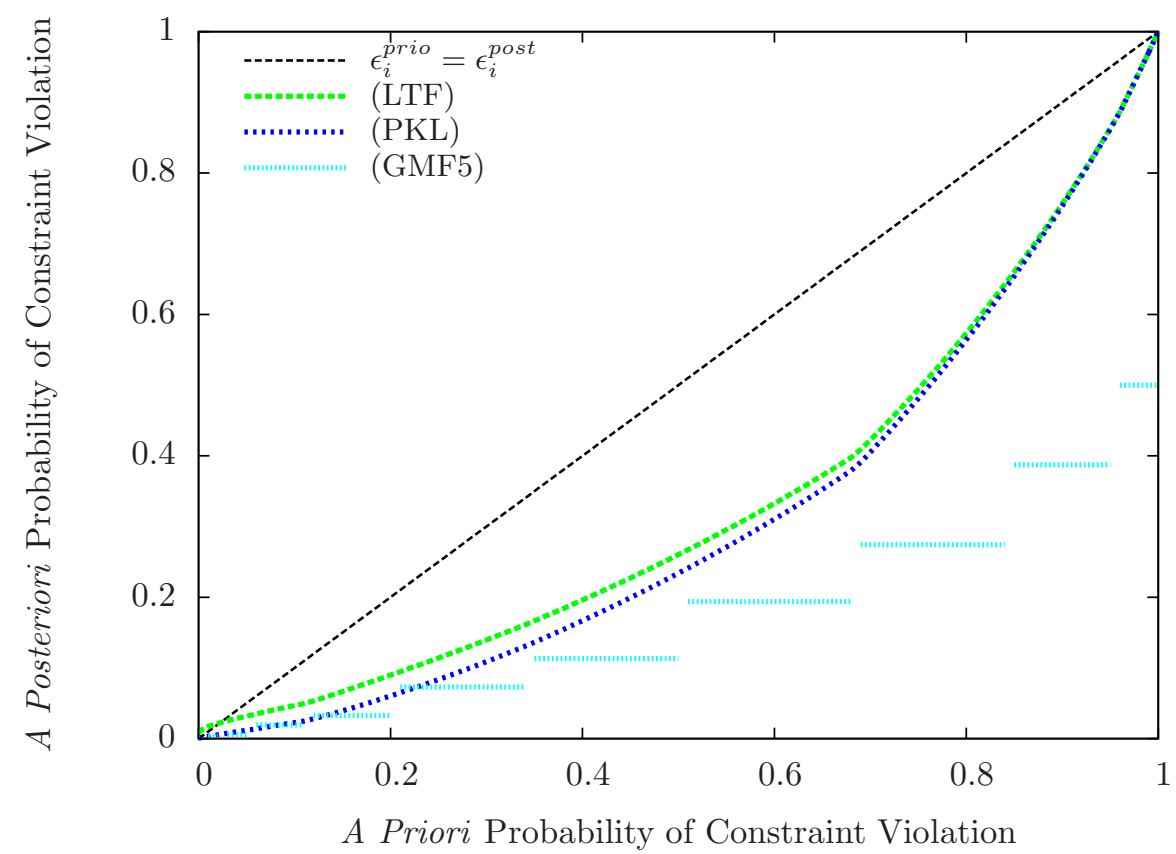

Figure 19: A posteriori probability of constraint violation of each solution obtained from the multiperiod planning problem with the interval + polyhedral uncertainty set versus a priori probability distribution; $\epsilon_{i}^{\text {prio }}$ was set by selecting $\Delta_{i}$ with bound (GMF2). 


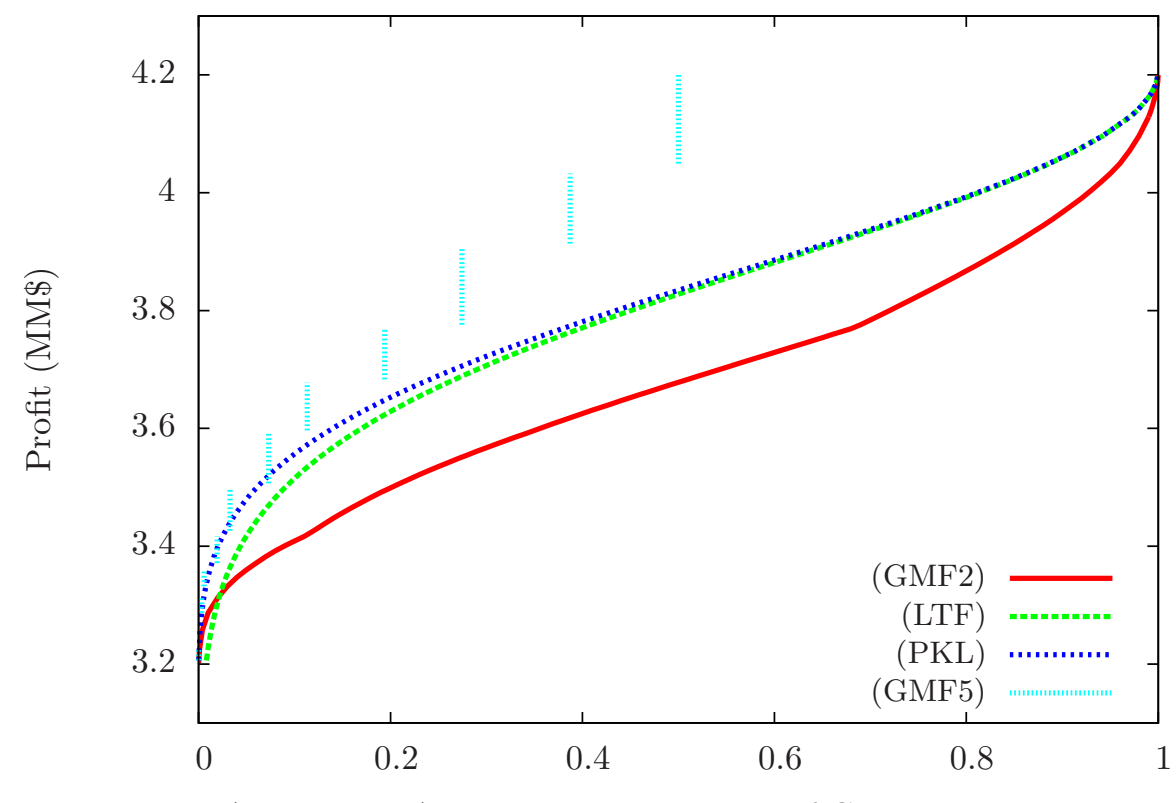

A Priori or A Posteriori Probability of Constraint Violation

Figure 20: Profit versus a posteriori probability of constraint violation of each solution obtained from the multiperiod planning problem with the interval + polyhedral uncertainty set and bound $\left(\mathrm{GMF}^{\prime}\right)$; profit versus $\epsilon_{i}^{\text {prio }}$ as obtained by bound (GMF2) included for comparison. 
Table 3: Comparison of existing and new a priori probabilistic bounds for the multiperiod production planning problem with unknown parameter distributions and an interval + polyhedral uncertainty set.

\begin{tabular}{|c|c|c|c|c|c|c|}
\hline \multirow[b]{3}{*}{$\epsilon_{i}^{\text {prio }}$} & \multicolumn{6}{|c|}{ Existing Bounds } \\
\hline & \multicolumn{3}{|c|}{ (BS1) } & \multicolumn{3}{|c|}{ (BS2) } \\
\hline & $\begin{array}{c}\text { Profit } \\
(\mathrm{MM} \$)\end{array}$ & $\begin{array}{c}\text { PoR } \\
(\%)\end{array}$ & $\begin{array}{c}\text { NPoR } \\
(\%)\end{array}$ & $\begin{array}{c}\text { Profit } \\
(\mathrm{MM} \$)\end{array}$ & $\begin{array}{c}\text { PoR } \\
(\%)\end{array}$ & $\begin{array}{c}\text { NPoR } \\
(\%)\end{array}$ \\
\hline 0.05 & 3.342 & 20.57 & 86.43 & 3.431 & 18.45 & 77.52 \\
\hline 0.10 & 3.395 & 19.30 & 81.10 & 3.544 & 15.78 & 66.28 \\
\hline 0.15 & 3.441 & 18.23 & 76.58 & 3.616 & 14.05 & 59.04 \\
\hline 0.20 & 3.487 & 17.13 & 71.99 & 3.687 & 12.36 & 51.95 \\
\hline 0.25 & 3.525 & 16.22 & 68.13 & 3.734 & 11.26 & 47.30 \\
\hline 0.30 & 3.559 & 15.41 & 64.74 & 3.785 & 10.04 & 42.18 \\
\hline \multirow[t]{2}{*}{0.35} & 3.590 & 14.68 & 61.67 & 3.857 & 8.34 & 35.06 \\
\hline & \multicolumn{6}{|c|}{ New Bounds } \\
\hline$\epsilon_{i}^{\text {prio }}$ & $\begin{array}{c}\text { Profit } \\
(\mathrm{MM} \$)\end{array}$ & $\begin{array}{c}\text { PoR } \\
(\%)\end{array}$ & $\begin{array}{c}\text { NPoR } \\
(\%)\end{array}$ & $\begin{array}{l}\text { Profit } \\
(\mathrm{MM} \$)\end{array}$ & $\begin{array}{c}\text { PoR } \\
(\%)\end{array}$ & $\begin{array}{c}\text { NPoR } \\
(\%)\end{array}$ \\
\hline 0.05 & 3.360 & 20.15 & 84.65 & 3.505 & 16.70 & 70.14 \\
\hline 0.10 & 3.409 & 18.98 & 79.76 & 3.592 & 14.64 & 61.51 \\
\hline 0.15 & 3.457 & 17.85 & 74.99 & 3.682 & 12.50 & 52.53 \\
\hline 0.20 & 3.499 & 16.84 & 70.75 & 3.772 & 10.36 & 43.54 \\
\hline 0.25 & 3.535 & 15.98 & 67.15 & 3.772 & 10.36 & 43.54 \\
\hline 0.30 & 3.567 & 15.22 & 63.95 & 3.910 & 7.09 & 29.77 \\
\hline 0.35 & 3.597 & 14.52 & 61.01 & 3.910 & 7.09 & 29.77 \\
\hline
\end{tabular}

Table 4: Definition of uncertainty sets.

\begin{tabular}{cclc}
\hline Uncertainty set $U_{i}$ & Parameter $\Delta_{i}{ }^{\mathrm{a}}$ & Definition & $\max \Delta_{i}^{\mathrm{b}}$ \\
\hline Box & $\Psi_{i}$ & $U_{i}^{\infty}=\left\{\xi_{i}:\left\|\xi_{i}\right\|_{\infty} \leq \Psi_{i}\right\}$ & 1 \\
Ellipsoidal & $\Omega_{i}$ & $U_{i}^{2}=\left\{\xi_{i}:\left\|\xi_{i}\right\|_{2} \leq \Omega_{i}\right\}$ & $\sqrt{\left|J_{i}\right|}$ \\
Interval + Ellipsoidal & $\Omega_{i}$ & $U_{i}^{2 \cap \infty}=\left\{\xi_{i}:\left\|\xi_{i}\right\|_{2} \leq \Omega_{i},\left\|\xi_{i}\right\|_{\infty} \leq 1\right\}$ & $\sqrt{\left|J_{i}\right|}$ \\
Polyhedral & $\Gamma_{i}$ & $U_{i}^{1}=\left\{\xi_{i}:\left\|\xi_{i}\right\|_{1} \leq \Gamma_{i}\right\}$ & $\left|J_{i}\right|$ \\
Interval + Polyhedral & $\Gamma_{i}$ & $U_{i}^{1 \cap \infty}=\left\{\xi_{i}:\left\|\xi_{i}\right\|_{1} \leq \Gamma_{i},\left\|\xi_{i}\right\|_{\infty} \leq 1\right\}$ & $\left|J_{i}\right|$ \\
\hline
\end{tabular}

${ }^{\text {a }}$ Original, set-specific notation of parameter denoted here; $\Delta_{i}$ used for the general case.

b Applicable when $\xi_{i j} \in[-1,1], \forall j \in J_{i}$. 
Table 5: Robust counterparts of constraint $i$ by uncertainty set type.

\begin{tabular}{ll}
\hline$U_{i} \quad$ & $\sum_{j} a_{i j} x_{j}+\max _{\xi_{i} \in U_{i}}\left\{\sum_{j \in J_{i}} \xi_{i j} \hat{a}_{i j} x_{j}\right\} \leq b_{i}$ \\
\hline $\mathrm{B} \quad$ & $\sum_{j} a_{i j} x_{j}+\Psi_{i} \sum_{j \in J_{i}} \hat{a}_{i j}\left|x_{j}\right| \leq b_{i}$ \\
$\mathrm{E}$ & $\sum_{j} a_{i j} x_{j}+\Omega_{i} \sqrt{\sum_{j \in J_{i}} \hat{a}_{i j}^{2} x_{j}^{2}} \leq b_{i}$ \\
$\mathrm{IE} \quad \sum_{j} a_{i j} x_{j}+\sum_{j \in J_{i}} \hat{a}_{i j}\left|x_{j}-z_{i j}\right|+\Omega_{i} \sqrt{\sum_{j \in J_{i}} \hat{a}_{i j}^{2} z_{j}^{2}} \leq b_{i}$ \\
$\mathrm{P} \quad(1) \sum_{j} a_{i j} x_{j}+\Gamma_{i} z_{i} \leq b_{i} \quad(2) z_{i} \geq \hat{a}_{i j}\left|x_{j}\right|, \forall j \in J_{i}$ \\
$\mathrm{IP} \quad(1) \sum_{j} a_{i j} x_{j}+\sum_{j \in J_{i}} p_{i j}+\Gamma_{i} z_{i} \leq b_{i} \quad(2) z_{i} \geq 0$ \\
\\
(3) $z_{i}+p_{i j} \geq \hat{a}_{i j}\left|x_{j}\right|, \forall j \in J_{i} \quad(4) p_{i j} \geq 0, \forall j \in J_{i}$
\end{tabular}

Table 6: Associated parameters of uncertainty sets. ${ }^{\text {a }}$

\begin{tabular}{llll}
\hline$U_{i}$ & Definition of $\delta_{i}$ & $\left\|\delta_{i}\right\|_{1} \leq \delta_{i}^{(1)}$ & $\left\|\delta_{i}\right\|_{2} \leq \delta_{i}^{(2)}$ \\
\hline $\mathrm{B}$ & $\delta_{i j}:=\frac{\hat{a}_{i j} x_{j}}{\sum_{j} \hat{a}_{i j}\left|x_{j}\right|}$ & $\sum_{j}\left|\delta_{i j}\right| \leq 1$ & $\sqrt{\sum_{j} \delta_{i j}^{2}} \leq 1$ \\
$\mathrm{E}$ & $\delta_{i j}:=\frac{\hat{a}_{i j} x_{j}}{\sqrt{\sum_{j} \hat{a}_{i j}^{2} x_{j}^{2} \mid}}$ & $\sum_{j}\left|\delta_{i j}\right| \leq \sqrt{\left|J_{i}\right|^{\mathrm{c}}}$ & $\sqrt{\sum_{j} \delta_{i j}^{2}}=1$ \\
$\mathrm{IE}$ & $\delta_{i j}:=\frac{\hat{a}_{i j} z_{j}}{\sqrt{\sum_{j} \hat{a}_{i j}^{2} z_{j}^{2} \mid}}$ & $\sum_{j}\left|\delta_{i j}\right| \leq \sqrt{\left|J_{i}\right|^{\mathrm{c}}}$ & $\sqrt{\sum_{j} \delta_{i j}^{2}}=1$ \\
$\mathrm{P}$ & $\delta_{i j}:=\frac{\hat{a}_{i j} x_{j}}{\max _{j} \hat{a}_{i j}\left|x_{j}\right|}$ & $\sum_{j}\left|\delta_{i j}\right| \leq\left|J_{i}\right|$ & $\sqrt{\sum_{j} \delta_{i j}^{2}} \leq \sqrt{\left|J_{i}\right|}$ \\
$\mathrm{IP} \mathrm{b}, \mathrm{d}$ & $\delta_{i j}:=\min \left\{1, \frac{\hat{a}_{i j}\left|x_{j}\right|}{\hat{a}_{i r}\left|x_{r}\right|}\right\}$ & $\sum_{j}\left|\delta_{i j}\right| \leq\left|J_{i}\right|$ & $\sqrt{\sum_{j} \delta_{i j}^{2}} \leq \sqrt{\left|J_{i}\right|}$ \\
\hline
\end{tabular}

${ }^{\text {a }}$ Note that all $j \in J_{i}$, and that for all uncertainty sets, $\delta_{i j} \in[-1,1]$. Notation uses vector $\delta_{i}$ with elements $\delta_{i j}$.

b Assumes that probability distributions are bounded.

${ }^{c}$ Follows from the Cauchy-Schwarz inequality.

${ }^{\mathrm{d}}$ Index $r$ is the index of the $\left(\max \left\{1,\left\lceil\Gamma_{i}\right\rceil\right\}\right)$ th largest $\hat{a}_{i j}\left|x_{j}\right|$. 
Table 7: Existing and new a priori probabilistic bounds on constraint violation: bounded distributions.

\begin{tabular}{|c|c|c|c|c|}
\hline Source & Label & Bound & Applicable $^{\mathrm{a}} U_{i}$ & Assumptions $^{\mathrm{b}}$ \\
\hline$\left({ }^{c}\right)$ & $(\mathrm{BN})$ & $\exp \left(-\Delta_{i}^{2} / 2\right)$ & $\mathrm{B}, \mathrm{E}, \mathrm{IE}$ & $\xi_{i j} \in[-1,1], \mathbf{E}\left[\xi_{i j}\right]=0$ \\
\hline$\left({ }^{d}\right)$ & $(\mathrm{BS} 1)$ & $\exp \left(-\Delta_{i}^{2} / 2\left|J_{i}\right|\right)$ & $\mathrm{B}, \mathrm{E}, \mathrm{IE}, \mathrm{P}, \mathrm{IP}$ & $\xi_{i j} \in[-1,1], \mathbf{E}\left[\xi_{i j}\right]=0$ \\
\hline $\begin{array}{l}\text { this } \\
\text { work }\end{array}$ & $(\mathrm{GMF} 1)^{\mathrm{e}}$ & $\exp \left(\min _{\theta>0}\left\{-\theta \Delta_{i}+\sum_{k \in K_{i}} \ln G_{i k}(\theta)\right\}\right)$ & $\mathrm{B}, \mathrm{E}, \mathrm{IE}, \mathrm{P}, \mathrm{IP}$ & $\xi_{i j} \in[-1,1]$, known bounds on $\mathbf{E}\left[\xi_{i j}\right]$ \\
\hline $\begin{array}{l}\text { this } \\
\text { work }\end{array}$ & $\left(\mathrm{GMF} 1^{\prime}\right)^{\mathrm{f}}$ & $\exp \left(\min _{\theta>0}\left\{-\theta \Delta_{i}+\left|J_{i}\right| \ln \bar{G}_{i}\left(\theta / \sqrt{\left|J_{i}\right|}\right)\right\}\right)$ & $\mathrm{E}, \mathrm{IE}$ & $\xi_{i j} \in[-1,1]$, known bounds on $\mathbf{E}\left[\xi_{i j}\right]$ \\
\hline $\begin{array}{l}\text { this } \\
\text { work }\end{array}$ & $(\mathrm{GMF} 2)^{\mathrm{g}}$ & $\left(\alpha^{\alpha} \beta^{\beta}\right)^{-\delta_{i}^{(1)} / 2}$ & $\mathrm{~B}, \mathrm{E}, \mathrm{IE}, \mathrm{P}, \mathrm{IP}$ & $\xi_{i j} \in[-1,1], \mathbf{E}\left[\xi_{i j}\right]=0$ \\
\hline $\begin{array}{l}\text { this } \\
\text { work }\end{array}$ & $\left(\mathrm{GMF} 2^{\prime}\right)^{\mathrm{g}}$ & $\left(\alpha^{\alpha} \beta^{\beta}\right)^{-\left|J_{i}\right| / 2}$ & $\mathrm{E}, \mathrm{IE}$ & $\xi_{i j} \in[-1,1], \mathbf{E}\left[\xi_{i j}\right]=0$ \\
\hline
\end{tabular}

${ }^{a}$ Bound (BN) was formulated for IE uncertainty sets, while bound (BS1) was formulated for IP uncertainty sets. Their usage was extended other uncertainty sets in (Li et al., 2012).

${ }^{\mathrm{b}}$ Bounds (BN) and (BS1) were derived under assumptions of symmetry; we note that they apply to cases where all $\mathbf{E}\left[\xi_{i j}\right]=0$.

c Ben-Tal and Nemirovski, 2000.

${ }^{\mathrm{d}}$ Bertsimas and Sim, 2004.

e $G_{i j}(\theta):=\mu_{i j} \sinh (\theta)+\cosh (\theta)$.

f $\bar{G}_{i}(\theta):=\left(\max _{j \in J_{i}} \mu_{i j}\right) \sinh (\theta)+\cosh (\theta)$

g $\alpha:=1-\Delta_{i} / \delta_{i}^{(1)}, \beta:=1+\Delta_{i} / \delta_{i}^{(1)}$. 
Table 8: Existing and new a priori probabilistic bounds on constraint violation: bounded, symmetric distributions. ${ }^{\text {a }}$

\begin{tabular}{|c|c|c|c|c|}
\hline Source & Label & Bound & Applicable $^{\mathrm{b}} U_{i}$ & Assumptions \\
\hline$\left({ }^{c}\right)$ & $(\mathrm{BS} 2)^{\mathrm{d}}$ & for $\Delta_{i} \geq 1, \frac{1}{2^{n}}\left[(1-(\nu-\lfloor\nu\rfloor))\left(\begin{array}{c}n \\
\lfloor\downarrow\rfloor\end{array}\right) i+\sum_{\substack{k=\lfloor\nu\rfloor+1 \\
n}}^{n}\left(\begin{array}{l}n \\
k\end{array}\right)\right.$ & $\mathrm{B}, \mathrm{E}, \mathrm{IE}, \mathrm{P}, \mathrm{IP}$ & $\xi_{i j} \in[-1,1], \mathbf{E}\left[\xi_{i j}\right]=0$, symmetric \\
\hline$(\mathrm{c})$ & $\left(\mathrm{BS} 2^{\prime}\right)^{\mathrm{d}, \mathrm{e}}$ & for $\Delta_{i} \geq 1,(1-(\nu-\lfloor\nu\rfloor)) C(n,\lfloor\nu\rfloor)+\sum_{k=\lfloor\nu\rfloor+1} C(n, k)$ & $\mathrm{B}, \mathrm{E}, \mathrm{IE}, \mathrm{P}, \mathrm{IP}$ & $\xi_{i j} \in[-1,1], \mathbf{E}\left[\xi_{i j}\right]=0$, symmetric \\
\hline $\begin{array}{l}\text { this } \\
\text { work }\end{array}$ & $(\mathrm{GMF} 3)^{\mathrm{f}}$ & $\frac{1}{2^{\tilde{n}}} \sum_{k=\lceil\tilde{\nu}\rceil}^{\tilde{n}}\left(\begin{array}{l}\tilde{n} \\
k\end{array}\right)$ & $\mathrm{B}, \mathrm{E}, \mathrm{IE}, \mathrm{P}, \mathrm{IP}$ & $\xi_{i j} \in[-1,1], \mathbf{E}\left[\xi_{i j}\right]=0$, symmetric \\
\hline $\begin{array}{l}\text { this } \\
\text { work }\end{array}$ & $\left(\mathrm{GMF}^{\prime}\right)^{\mathrm{e}, \mathrm{f}}$ & $\sum_{k=\lceil\tilde{\nu}\rceil}^{\tilde{n}} C(\tilde{n}, k)$ & B, E, IE, P, IP & $\xi_{i j} \in[-1,1], \mathbf{E}\left[\xi_{i j}\right]=0$, symmetric \\
\hline
\end{tabular}

a The bounds of Table 7 also apply.

${ }^{\mathrm{b}}$ Bounds (BS2) and (BS2') were formulated for IP uncertainty sets. Their usage was extended other uncertainty sets in (Li et al., 2012)

c Bertsimas and Sim, 2004.

d $\nu:=\left(\Delta_{i}+n\right) / 2, n:=\left|J_{i}\right|$.

e Less computationally expensive for large $\left|J_{i}\right|$ with slight loss of tightness.

f $\tilde{\nu}:=\left(\Delta_{i}+\tilde{n}\right) / 2, \tilde{n}:=\left|J_{i}\right|+1-\operatorname{sgn} \Delta_{i}$ or $\left|J_{i}\right|-\operatorname{sgn} \Delta_{i}$. 
Table 9: Tightest a priori bounds by case. ${ }^{\mathrm{a}}$

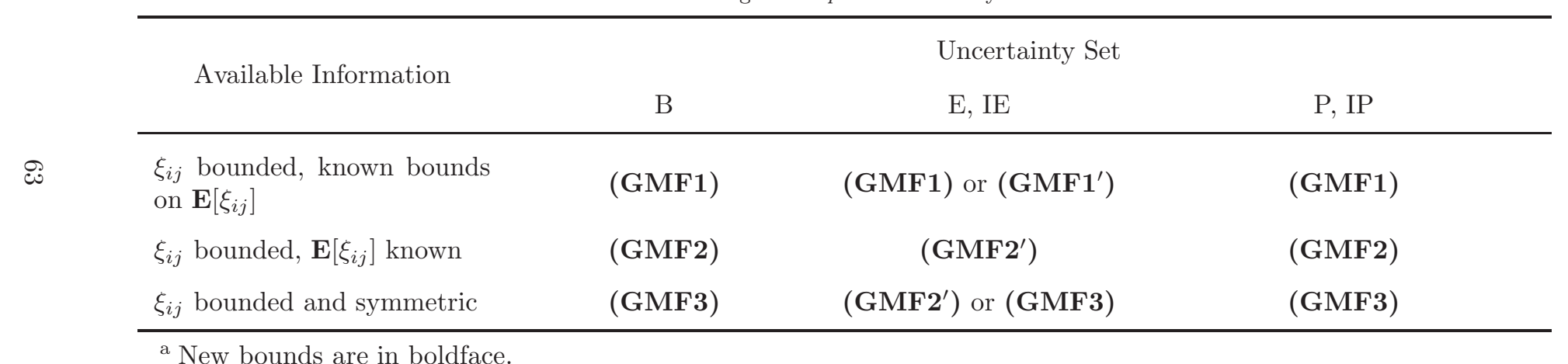

a New bounds are in boldface. 
Table 10: Existing and new a posteriori probabilistic bounds on constraint violation.

\begin{tabular}{|c|c|c|c|c|}
\hline Source & Label & \multicolumn{2}{|c|}{ Bound } & Assumptions \\
\hline $\begin{array}{l}\text { this } \\
\text { work }\end{array}$ & $(\mathrm{GMF} 4)^{\mathrm{a}, \mathrm{b}}$ & $\exp$ & $\min _{\theta>0}\left\{-\theta h_{i}\left(x^{*}\right)+\sum_{j \in J_{i}} \ln G_{i j}\left(\left|\hat{a}_{i j} x_{j}^{*}\right| \theta\right)\right.$ & $\xi_{i j} \in[-1,1]$, known bounds on $\mathbf{E}\left[\xi_{i j}\right]$ \\
\hline$\left({ }^{c}\right)$ & $(\mathrm{LTF})^{\mathrm{a}}$ & $\exp$ & $\left(\frac{-h_{i}\left(x^{*}\right)^{2}}{2 \sum_{j \in J_{i}} \hat{a}_{i j}^{2} x_{j}^{* 2}}\right)$ & $\xi_{i j} \in[-1,1], \mathbf{E}\left[\xi_{i j}\right]=0$ \\
\hline$(\mathrm{d})$ & $(\mathrm{PKL})^{\mathrm{a}}$ & $\exp$ & $\min _{\theta>0}\left\{-\theta h_{i}\left(x^{*}\right)+\sum_{j \in J_{i}} \ln \cosh \left(\hat{a}_{i j} x_{j}^{*} \theta\right)\right.$ & $\xi_{i j} \in[-1,1], \mathbf{E}\left[\xi_{i j}\right]=0$ \\
\hline $\begin{array}{l}\text { this } \\
\text { work }\end{array}$ & $(\text { GMF5 })^{\mathrm{e}, \mathrm{f}}$ & $\min$ & $\left\{\frac{1}{2^{n_{a}}} \sum_{k=\left\lceil\nu_{a}\right]}^{n_{a}}\left(\begin{array}{c}n_{a} \\
k\end{array}\right), \frac{1}{2^{n_{b}}} \sum_{k=\left\lceil\nu_{b}\right\rceil}^{n_{b}}\left(\begin{array}{c}n_{b} \\
k\end{array}\right)\right\}$ & $\xi_{i j} \in[-1,1], \mathbf{E}\left[\xi_{i j}\right]=0$, symmetric \\
\hline $\begin{array}{l}\text { this } \\
\text { work }\end{array}$ & $\left(\mathrm{GMF}^{\prime}\right)^{\mathrm{e}, \mathrm{f}, \mathrm{g}}$ & $\min$ & $\left\{\sum_{k=\left\lceil\nu_{a}\right\rceil}^{n_{a}} C\left(n_{a}, k\right), \sum_{k=\left\lceil\nu_{b}\right\rceil}^{n_{b}} C\left(n_{b}, k\right)\right\}$ & $\xi_{i j} \in[-1,1], \mathbf{E}\left[\xi_{i j}\right]=0$, symmetric \\
\hline
\end{tabular}

${ }^{\mathrm{a}} h_{i}(x):=b_{i}-\sum_{j} a_{i j} x_{j}$.

${ }^{\mathrm{b}} G_{i j}(\theta):=\mu_{i j} \sinh (\theta)+\cosh (\theta)$.

${ }^{\mathrm{c}} \mathrm{Li}$ et al., 2012.

${ }^{\mathrm{d}}$ Paschalidis et al., 2008.

e $\nu_{a}:=\left(h_{a}+n_{a}\right) / 2, n_{a}:=\left|J_{i}^{\delta, *}\right|+1-\operatorname{sgn} h_{a}$ or $\left|J_{i}^{\delta, *}\right|-\operatorname{sgn} h_{a}, h_{a}:=\Delta_{i} /\left(\max _{j \in J_{i}^{\delta, *}}\left|\delta_{i j}^{*}\right|\right), J_{i}^{\delta, *}:=\left\{j:\left|\delta_{i j}^{*}\right|>0\right\}$.

${ }^{\mathrm{f}} \nu_{b}:=\left(h_{b}+n_{b}\right) / 2, n_{b}:=\left|J_{i}^{*}\right|+1-\operatorname{sgn} h_{b}$ or $\left|J_{i}^{*}\right|-\operatorname{sgn} h_{b}, h_{b}:=h_{i}\left(x^{*}\right) /\left(\max _{j \in J_{i}^{*}} \hat{a}_{i j}\left|x_{j}^{*}\right|\right), J_{i}^{*}:=\left\{j: \hat{a}_{i j}\left|x_{j}^{*}\right|>0\right\}$.

${ }^{\mathrm{g}}$ Less computationally expensive for large $\left|J_{i}^{\delta, *}\right|$ or $\left|J_{i}^{*}\right|$ with slight loss of tightness. 


\section{Acknowledgments}

The authors gratefully acknowledge financial support from the National Science Foundation (NSF CBET-1158849). LRM gratefully acknowledges a National Defense Science and Engineering Graduate (NDSEG) fellowship.

\section{References}

\section{References}

Ben-Tal, A., Nemirovski, A., 1998. Robust convex optimization. Mathematics of Operations Research 23 (4), 769-805.

Ben-Tal, A., Nemirovski, A., 1999. Robust solutions of uncertain linear programs. Operations Research Letters 25 (1), 1-13.

Ben-Tal, A., Nemirovski, A., 2000. Robust solutions of linear programming problems contaminated with uncertain data. Mathematical Programming 88 (3), 411-424.

Bennett, G., 1962. Probability inequalities for the sum of independent random variables. Journal of the American Statistical Association 57 (297), 33-45.

Bertsimas, D., Sim, M., 2004. The price of robustness. Operations Research $52(1), 35-53$.

Dembo, A., Zeitouni, O., 2009. Large deviations techniques and applications. Vol. 38. Springer Science \& Business Media.

Dzindzalieta, D., Juskevicius, T., Sileikis, M., 2012. Optimal probability inequalities for random walks related to problems in extremal combinatorics. SIAM Journal on Discrete Mathematics 26 (2), 828-837.

El Ghaoui, L., Lebret, H., 1997. Robust solutions to least-squares problems with uncertain data. SIAM Journal on Matrix Analysis and Applications 18 (4), $1035-1064$.

El Ghaoui, L., Oustry, F., Lebret, H., 1998. Robust solutions to uncertain semidefinite programs. SIAM Journal on Optimization 9 (1), 33-52. 
Falk, J. E., Hoffman, K. R., 1976. A successive underestimation method for concave minimization problems. Mathematics of Operations Research 1 (3), $251-259$.

Hoeffding, W., 1963. Probability inequalities for sums of bounded random variables. Journal of the American Statistical Association 58 (301), 13-30.

Janak, S. L., Lin, X., Floudas, C. A., 2007. A new robust optimization approach for scheduling under uncertainty: II. Uncertainty with known probability distribution. Computers \& Chemical Engineering 31 (3), 171-195.

Kang, S.-C., Brisimi, T. S., Paschalidis, I. C., 2013. Distribution-dependent robust linear optimization with applications to inventory control. Annals of Operations Research, 1-35.

Li, Z., Ding, R., Floudas, C. A., 2011. A comparative theoretical and computational study on robust counterpart optimization: I. Robust linear optimization and robust mixed integer linear optimization. Industrial \& Engineering Chemistry Research 50 (18), 10567-10603.

Li, Z., Floudas, C. A., 2014. A comparative theoretical and computational study on robust counterpart optimization: III. Improving the quality of robust solutions. Industrial \& Engineering Chemistry Research 53 (33), 13112-13124.

Li, Z., Tang, Q., Floudas, C. A., 2012. A comparative theoretical and computational study on robust counterpart optimization: II. Probabilistic guarantees on constraint satisfaction. Industrial \& Engineering Chemistry Research 51 (19), 6769-6788.

Lin, X., Janak, S. L., Floudas, C. A., 2004. A new robust optimization approach for scheduling under uncertainty:: I. Bounded uncertainty. Computers \& Chemical Engineering 28 (6), 1069-1085.

Paschalidis, I., Kang, S., Li, K., 2008. Distribution-dependent robust linear optimization with asymmetric uncertainty and application to optimal control. In: Proceedings of the 17th World Congress IFAC.

Ravindran, A. R., 2008. Operations Research Methodologies. CRC Press.

Soyster, A. L., 1973. Convex programming with set-inclusive constraints and applications to inexact linear programming. Operations Research 21 (5), 11541157 .

Tardella, F., 2003. On the existence of polyhedral convex envelopes. In: Floudas, C. A., Pardalos, P. M. (Eds.), Frontiers in Global Optimization. Kluwer Academic Publishers, pp. 563-573.

Verderame, P. M., Floudas, C. A., 2009a. Operational planning of large-scale industrial batch plants under demand due date and amount uncertainty. i. robust optimization framework. Industrial \& Engineering Chemistry Research 48 (15), 7214-7231. 
Verderame, P. M., Floudas, C. A., 2009b. Operational planning of large-scale industrial batch plants under demand due date and amount uncertainty: II. Conditional value-at-risk framework. Industrial \& Engineering Chemistry Research 49 (1), 260-275. 